\title{
ATTRIBUTIONAL THEORY, ORGANISATIONAL CULTURE AND MOTIVATION
}

\author{
A thesis submitted to the \\ Faculty of Science \\ of the University of London \\ for the degree of Doctor of Philosophy
}

Athena Xenikou

Department of Psychology

University College London

December 1995 


\section{Acknowledgements}

First and foremost, I wish to thank my supervisor Professor Adrian Furnham for his continuous support and scientific insight. His door was always open indicating that whenever there was a problem he was willing to help me out.

I am grateful to Professor Alexandra Hantzi, Professor Theoni Velli, and Professor Aristotelis Kantas for being supportive on numerous occasions.

For three years of financial sponsorship, I am indebted to the State Scholarships Foundation. I would also like to thank the foundation "Lilian Boudouri" for awarding me an one-year scholarship. Finally, I am indebted to the people who participated in my studies giving generously of their time.

Finally, my thanks go to the U.C.L. Department of Psychology as part of which I had the opportunity to carry out my research project. The friendliness of my colleagues in the Department has helped me to get through this last year. 
To my family, Maria, Stamatis and Stelios. 


\section{Abstract}

This thesis concerns the effect of attributions for failing in a creativity task and organisational culture $(\mathrm{OC})$ on motivation to engage in similar tasks.

In chapter one the relationship between situational attributions, attributional style (AS), and motivation is reviewed. A reformulation of Amabile's model of the social psychology of creativity is suggested on the grounds of recent developments in attributional theory. An extension of Amabile's theory is also proposed by investigating various social facilitators of creativity. In order for the proposed extension of Amabile's theory to be further elaborated within the organisational setting, the effect of organisational culture on creativity and innovation is reviewed in chapter two.

Five studies were conducted to test a series of hypotheses derived from the above research. In study one, the moderating role of situational attributions in the relationship between failure and subsequent motivation was empirically shown in terms of the refined attributional theory of Weiner.

Since the literature in AS has questioned the psychometric properties of the various measures of the concept, study two concerns the development of a more reliable and valid measure of AS. Results showed that a generalised expectancy for negative events is a predictor of low confidence and pessimism.

In study three the new measure of AS was used to test for the hypothesised influence of AS on after-failure motivation through its effect on situational attributions. The suggested extension of Amabile's social psychology of creativity was also tested by examining whether pro-creativity social norms facilitate creative behaviour. The findings demonstrate that the globality facet of AS and the perceived social norms for creativity determine the perception of situational attributions, which in turn predicts the level of after-failure motivation.

In order to examine the effect of social norms on motivation to be creative in the organisational setting, organisational norms as a manifestation of $\mathrm{OC}$ had to be measured. The fourth study was a psychometric assessment of four questionnaire measures of $\mathrm{OC}$ which showed the more reliable and valid measure to use. In addition, study four provided some evidence that the organisational norms of creativity, internal co-operation, and achievement constitute the cultural dimension of openness to change, while the norms of centralisation of power and competition are associated with resistance to change. 
The final study investigated the effect of $\mathrm{OC}$ on employees motivation to be creative through the mediating effect of situational attributions for failure and expectancy of future success. The hypotheses of this study were partly supported.

The final chapter discusses the findings and the limitations of this thesis, drawing out possible implications for future research.

Note. Study two has been accepted for publication in the British Journal of Psychology and study four in Human Relations. Study three is currently under editorial review by the British Journal of Psychology. 


\section{TABLE OF CONTENTS}

CHAPTER

Overview

ONE

CHAPTER

TWO

Part I

2.1 .

2.1.1

2.1.2

2.1.3

2.2

2.2.1
Attributions for failure and the motivation

to be creative

and measurement of attributional style

The effect of situational attributions and

attributional style on motivation

Part II

A reformulation and extension of Amabile's social

psychology of creativity 
2.1 A contribution of attributional theory to the reformulation of Amabile's social psychology of creativity ........................................................... 33

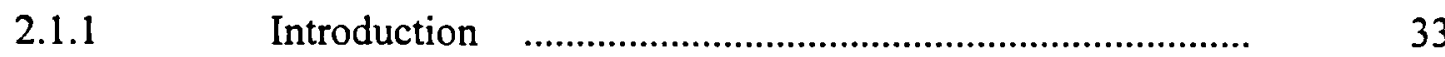

2.1.2 Amabile's model of the social psychology of creativity

2.1.3 An attributional critique and reformulation of Amabile's creativity model An extension of Amabile's theory: Pro-creativity social norms as a facilitator of creative behaviour

CHAPTER THREE

3.3 .2

\section{Creativity /innovation at the workplace and} organisational culture

Organisational culture $\quad$......................................... 54

Definition of organisational culture $\quad$................................ $\quad 55$

Elements of organisational culture $\quad$.................................. $\quad 57$

Organisational culture and organisational climate $\quad$............ $\quad 59$ 
3.4 The relationship between organisational

culture/climate and organisational innovation

3.4.1 The effect of bureaucratic aspects of organisational

culture on innovation

3.4.2 The enhancement of innovation by a culture/climate

that values creativity, achievement, and internal

co-operation

3.5 The extension of Amabile's creativity theory within

organisational behaviour

Introduction

Method

Respondents

Achievement Task

Questionnaires

Procedure

Results

Discussion 
CHAPTER

FIVE

CHAPTER

$S I X$
A proposition for a more reliable and valid

measure of attributional style

Introduction

98

Method

105

Respondents

105

Questionnaires

106

Procedure

109

Results

109

Discussion

A reformulation and extension of Amabile's

creativity model based on attributional

theory

A description of Amabile's model of creativity

A critique of Amabile's model based on

attributional theory

Social factors that facilitate creative behaviour

Method

Respondents

Questionnaires

Procedure

Results 136

Discussion 
CHAPTER

SEVEN

A correlational and factor analytic study of four

questionnaire measures of organisational culture

Introduction

The issue of the research methods in the study of

organisational culture

Quantitative measures of organisational culture

Aim and hypotheses of the current study

Method

Respondents

Questionnaires

162

Procedure

164

Results

Discussion

The extension of Amabile's creativity theory

Introduction

Method

Respondents

Questionnaires

Procedure

Results

Discussion 
Introduction

Study 2

9.3 Situational attributions for failure and subsequent motivation

9.3.2 Social learning theory vs. the refined attributional theory of Weiner

9.3.3 The issue of the measurement of causal stability

9.4 Situational attributions, attributional style, and motivation

9.4.1 The conceptualisation and measurement of attributional style

9.4.2 The relation between situational attributions, attributional style, and motivation

9.5 The attributional reformulation of Amabile's creativity model

9.6 The extension of Amabile's "social psychology

of creativity" 


\section{LIST OF TABLES}

Table 4.1 Descriptive statistics of locus of control (LC), causal stability (CS), and afterfailure motivation (AFM) for the whole sample, the subgroup of high initial motivation (SHIM), and the subgroup of low initial motivation (SLIM).

Table 4.2 Regression analysis of after-failure motivation on locus of control (Criterion: Scores on after-failure motivation).

Table 4.3 Regression analysis of after-failure motivation on causal stability (Criterion: Scores on after-failure motivation).

Table 4.4 Regression analysis of after-failure motivation on causal stability (Criterion: Scores on after-failure motivation) for the subgroup of high initial motivation.

Table 4.5 Regression analysis of after-failure motivation on causal stability (Criterion: Scores on after

Table 4.6 Regression analysis of after-failure motivation on locus of control (Criterion: Scores on after-failure motivation) for the subgroup of high initial motivation.

Table 4.7 Regression analysis of after-failure motivation on locus of control (Criterion: Scores on after-failure motivation) for the subgroup of low initial motivation.

Table 5.1 Descriptive statistics, alphas, and zero-order correlation coefficients between the OASQ's scales.

Table 5.2 Factor analysis of the OASQ's scales of internality, stability, and globality for positive and negative events.

Table 5.3 Studies in attributional style reporting alpha coefficients of internal consistency that show the same pattern of results.

Table 5.4 Pearson correlations among negative stability (NS), negative globality (NG), NPO, and RPQ's subscale of confidence (CON).

Table 5.5 Multiple regression of the NPO scale (Criterion: Scores on the NPO scale).

Table 5.6 Multiple regression of the RPQ's scale of confidence (Criterion: Scores on the confidence scale).

Table 6.1 Items that comprise the CGS "Task Innovation" scale.

Table 6.2 Hypothetical negative events used to measure negative stability and globality.

Table 6.3 Observed frequencies, expected frequencies, and residuals of shift in motivation 
measured on two 7-point scales.

Table 6.4 Pearson correlations among causal stability (CS), social norms concerning creativity (SNCC), attributional style's dimension of stability (ASDS), attributional style's dimension of globality (ASDG), intrinsic task motivation (ITM), and the demographic variables (sex, age, and year of studies).

Table 6.5 Hierarchical multiple regression. Dependent variable: Shift in intrinsic motivation.

Table 6.6 Hierarchical multiple regression. Dependent variable: Causal stability of failure.

Table 6.7 Regression of causal stability of failure on social norms for creativity and negative globality.

Table 6.8 Multiple regression. Dependent variable: Shift in intrinsic motivation.

Table 6.9 Multiple regression. Dependent variable: Causal stability of failure.

Table 7.1 Predicted correlations among overlapping subscales of OCI, CGS, and OBQ.

Table 7.2 Alpha reliabilities, descriptive statistics, and intercorrelations of the OCI subscales.

Table 7.3 Alpha reliabilities, descriptive statistics, and intercorrelations of the CGS subscales.

Table 7.4 Alpha reliabilities, descriptive statistics, and intercorrelations of the $O B Q$ subscales.

Table 7.5 Alpha reliabilities, descriptive statistics, and intercorrelations of the CCS subscales.

Table 7.6 Partial correlations among the overlapping subscales of the OCI, the CGS, and the OBQ with age, hierarchical level, and years of employment in the organisation partialled out.

Table 7.7 Factor analysis of the culture inventories subscales.

Table 8.1 Pearson correlations among shift in intrinsic motivation (SIM), expectancy of success (ES), causal stability (CS), openness to change (OC), and resistance change (RC).

Table 8.2 Multiple regression analysis. Dependent variable: Shift in intrinsic motivation.

Table 8.3 Multiple regression analysis. Dependent variable: Causal stability.

Table 8.4 Multiple regression analysis. Dependent variable: Expectation of success.

Table 8.5 Multiple regression analysis. Dependent variable: Shift in intrinsic motivation. 


\section{LIST OF FIGURES}

Figure 2.1 The componential model of creativity (Amabile, 1983).

Figure 2.2 A proposition for the reformulation and extension of Amabile's model in the case of failure.

Figure 3.1 Elements of organisational culture (Rousseau, 1990).

Figure 6.1 Graph showing the hypothesised relations between the model variables.

Figure 6.2 A model of the effect of failure in a creativity task on intrinsic task motivation.

Figure 6.3 The assessment of the model using Linear Structural Relationships (Jöreskog \& Sörbom, 1993).

Figure 8.1 Graph showing the correspondence of hypotheses and paths.

Figure 8.2 The indirect effect of organisational norms on shift in intrinsic motivation via expectation of future success. 
CHAPTER ONE

\section{OVERVIEW}


This thesis concerns the moderating effect of attributions in the relation between failure in a task and the levels of subsequent motivation. Social learning theory (Rotter, 1966), as well as Weiner's (1986) attributional theory, have suggested that attributions are important factors that determine the influence of failure or success on subsequent motivation. The difference between social learning theory and Weiner's attributional theory is that the former identified the attributional locus of control as the variable that predicts subsequent levels of motivation, whereas the latter suggested that the best predictor of motivation is the stability of the attributions. The experimental comparison of the two theories supported the theoretical predictions of Weiner's attributional theory, which is a more successful paradigm for the investigation of the effect of attributions on after-failure motivation.

Various studies, however, failed to fully confirm or contradicted Weiner's attributional theory of achievement motivation (Covington \& Omelich, 1979; 1984; Graham, 1984; Dalal \& Sethi, 1988; Winefield, Tiggemann, \& Winefield, 1992). After a thorough examination of the results of these studies, this thesis argues that the predictive power of causal stability as a moderator of the effect of failure on subsequent motivation will be improved by taking into account the initial level of the person's motivation to engage in the task. It is hypothesised that causal stability is a good predictor of after-failure motivation to engage in similar tasks provided that the initial interest to engage in the task is high. If a person fails in a task and attributes his/her failure to causes that are changeable, he/she will make an effort to change these causes only if there is interest in succeeding. Therefore, causal stability (the likelihood that causes of events can change) is a major predictor of after-failure motivation, as far as the person is interested in altering the causes of failure which are potentially changeable. 
In addition, the joint effect of attributions for a single event of failure and attributional style on a person's after-failure motivation to engage in similar tasks is examined. Recent research has reported that situational attributions have a more dominant effect on subsequent motivation than attributional style (Anderson, 1983; Mikulincer, 1990). More specifically, attributional style was shown to be a good predictor of after-failure motivation (pessimistic attributional style was related to lower motivation, while optimistic attributional style was related to higher motivation) given that there was no information leading to specific situational attributions. On the other hand, when experimentally manipulated information leading to specific situational attributions was provided, attributional style did not predict the levels of after-failure motivation. For example, informing a person that he/she failed to convince enough others to become blood donors because he/she did not use the right strategy (changeable cause) or does not have the charisma to persuade people (unchangeable cause) eliminates any effect of attributional style on after-failure motivation (experimental manipulation of situational attributions used by Anderson in 1983).

In this thesis it is argued that the competing relationship between attributional style and situational attributions can be the result of the fact that situational information provided by the experimenter is in fact indisputable. If the situational information leading to the formulation of situational attributions is ambiguous, then it is possible that attributional style effects the perception of this information and thereafter, the process of making situational attributions.

To test the hypotheses concerning firstly, whether attributions moderate the effect of failure on motivation; secondly, whether taking into account the initial level of motivation for a task improves the predictive validity of causal stability; and thirdly, whether attributional 
style influences the formulation of situational attributions when there is ambiguous situational information, the framework of the creativity process was utilised. This framework was selected primarily on the basis that Amabile's (1983) model of the social psychology of creativity suggests that when the outcome of the creativity process is failure, motivation for similar tasks decreases. This suggestion completely disregards the fact that attributions may moderate the effect of failure on subsequent motivation. One of the aims of this thesis was to test for the relationship between attributions and after-failure motivation, and on the basis of the findings to propose a reformulation of Amabile's model of creativity in order to incorporate attributions as the moderator of the effect of failure on subsequent motivation to be creative.

Another aim was to extend Amabile's theory of creativity by investigating social factors that might facilitate creative behaviour. It is suggested that in the case of failing in a creativity task social norms for creativity might influence subsequent motivation for similar tasks through their effect on the formulation of situational attributions. In other words, people who are members of groups which have pro-creativity social norms might be inclined to attribute their failure to causes that lead to the maintenance/increase of the levels of motivation, while members of groups with anti-creativity norms might tend to make attributions for failure which impair motivation.

The process of social comparison can be used to explain why such a phenomenon might occur. Groups that promote creativity often encourage risk taking, which leads to experiencing a considerable number of failures as well as successes. On the other hand, groups that have anti-creativity (risk-aversive) norms value failure avoidance rather than innovation. Therefore, there is a possibility that failure is more frequently encountered in the 
pro-creativity social groups compared to the anti-creativity social groups. According to the social comparison literature, when people encounter a threatening event such as failure they tend to compare themselves with equal or less advantaged others (Wood, 1989; Levine \& Green, 1984). In the case of failing they would look for other members of the group who experienced failure in a creativity task. If the group has pro-creativity norms there is going to be a considerable number of colleagues' failures that can be used for the social comparison and thus failure might be attributed to a changeable factor that will not necessarily repeat itself. On the other hand, when the group discourages creativity there are probably less instances of failing, the comparison with equal others becomes more difficult, and a tendency to explain negative events by the use of unchangeable causes appears.

Finally, the extension of Amabile's creativity theory is further elaborated within organisational behaviour. The effect of social norms on after-failure motivation can be more thoroughly examined within organisational behaviour since there has been a considerable effort spent by organisational theorists in trying to conceptualise and measure organisational norms, values, and beliefs which form the construct of organisational culture (Cooke \& Lafferty, 1989; Kilman \& Saxton, 1983; Sashkin, 1984; Glaser, 1983).

This thesis, therefore, focuses on the effect of situational attributions for failure and attributional style on after-failure motivation. The findings are used for the reformulation of Amabile's creativity model, while an extension of the social psychology of creativity is also suggested by the investigation of social factors that might promote creative behaviour. The proposed extension of the social psychology of creativity is further elaborated within organisational behaviour. 


\section{CHAPTER TWO}

\section{ATTRIBUTIONS FOR FAILURE AND THE MOTIVATION TO BE CREATIVE}




\section{PART I \\ The effect of situational attributions \\ and attributional style}

on motivation 


\subsection{SITUATIONAL ATTRIBUTIONS AND MOTIVATION.}

In the following four sections the effect of situational attributions on motivation will be discussed. The term situational attributions refer to the attributions that are provided for explaining the occurrence of a specific event. The relationship of situational attributions and motivation has been extensively examined within the paradigms of Social learning theory and Weiner's attributional theory.

\subsubsection{Social Learning Theory and motivation.}

According to social learning theory (Rotter, Seeman, \& Liverant, 1962; Rotter, 1966), a reinforcement (reward, positive outcome) acts to strengthen an expectancy that a particular behaviour will be followed by that reinforcement in the future. If a learning situation has occurred, in which a behaviour-reinforcement sequence has been established and the reinforcement is subjectively valued by the person, he/she will be motivated to engage in the same or similar behaviours, because of the expectancy that the behaviour will be followed by the reinforcement. Once an expectancy for such behaviour-reinforcement sequence is built up the failure of the reinforcement to occur will reduce or extinguish the expectancy. For example, a person who is looking for an unusual brand of tobacco and is finally able to find it will return to the same place were he/she was reinforced before, when he/she needs tobacco again. However, if he/she tries to find tobacco in that same shop and it is not available, then this behaviour will reduce and, after a couple of trials, will extinguish.

The social learning theorists, however, have recognised and further illustrated that the 
effect of reinforcement differs whether the task concerns skill or chance. When the person is engaged in a skill task, the occurrence of reinforcement after a specific behaviour raises expectancy that in the future behaviour will be followed by the reinforcement, while the absence of reinforcement weakens the expectancy of behaviour-reinforcement sequence (typical expectancy shifts). On the other hand, under chance conditions we observe "the gambler's fallacy" which refers to a belief that loss is expected after winning and a win is anticipated after losing. Therefore, chance tasks differ from skill tasks on the basis of the finding that they cause an effect opposite to the usual effect of reinforcement; that is, the occurrence of the reinforcement leads to lower expectations for future behaviourreinforcement sequence, while its absence is followed by higher expectations for subsequent behaviour-reinforcement sequence (atypical expectancy shifts).

Within social leaning theory, a number of studies have demonstrated that in skill tasks typical expectancy shifts were more frequent and of greater magnitude, while atypical expectancy shifts were more often observed in chance tasks (loss is expected after winning and a win is anticipated after losing). Phares (1957) used colour or line matching as an ambiguous task and instructed half of the subjects that the task was so difficult as to be a matter of luck and the other half of his subjects that success was a matter of skill (visual acuity). The research participants had to determine whether two colours or two lines were identical. A fixed order of partial reinforcement (right or wrong) was used and the measure of expectancy was the number of chips a participant would bet on his/her probability of being correct on the next trial. The data revealed that typical expectancy shifts were more frequent and of greater magnitude in the skill condition, whereas atypical expectancy shifts were more evident in the chance condition. However, there were some observations of atypical shifts in 
skill tasks and of typical shifts in the chance tasks. Holden and Rotter (1962) used an extrasensory perception type of task instructing one group of participants that it was a skill task and another group that it was determined entirely by luck. A third group of participants were given ambiguous instructions. Partial reinforcement was provided and subjects were given two dollars in nickels and told they could bet a nickel on each trial on whether or not they expected to succeed until they wished to discontinue and keep the rest of the money or ran out of money. Results showed that in the chance condition and in the ambiguous instructions condition, it took significantly more trials for the subjects to quit the experiment (extinction).

To explain these findings Rotter and his colleagues suggested that expectancy change following success or failure and the magnitude of motivation to engage in the same or a similar behaviour are influenced by the perception of causes as internally or externally controlled. When one perceives that the reward is contingent upon his/her own behaviour or attributes (skill task), there is higher expectation of future success after a successful outcome and lower expectation after failure. On the other hand, if the causes of an event are perceived as controlled by forces outside one's self (chance task), expectancies of goal attainment tend to decrease after success and to increase after failure. In other words, typical expectancy shifts occur when attributions are perceived as internally controlled, while atypical expectancy shifts are the result of perceiving reward as externally controlled. Therefore, it was concluded that typical shifts in skill tasks occur because of the attribution of the outcome to internally controlled causes, whereas atypical shifts following chance tasks are caused by the attribution of the outcome to externally controlled factors.

In conclusion, the expectancy change and the motivation following a success or a 
failure is influenced by the perceived locus of control; more specifically, perceived internal or personal control strengthens the behaviour-reinforcement sequence, while external or environmental control does not.

According to social learning theory, when people perceive their behaviour or attributes as contingent upon the attainment of a reward and thus, that there is a causal relation between their behaviour or attributes and the occurrence of the reward, motivation to engage in the task is affected by the absence or presence of the reinforcement. On the other hand, if the person does not believe that there is a causal link between his/her action or predisposition and the reward, the absence or presence of the reinforcement will not have such an influence on motivation to engage in the specific or similar tasks.

\subsubsection{Weiner's Attributional Theory of Achievement Motivation.}

Weiner's (1986) attributional theory of motivation suggests that when an outcome is unexpected, important, and /or involves nonattainment of a desired goal a causal search is likely to be immediately undertaken. An important set of variables that determine the results of this search is information about consensus, distinctiveness, and consistency as presented in Kelley's (1967) model, which are labelled by Brewin and Furnham (1986) as preattributional variables. Preattributional variables differ from attributional dimensions as the former refer to the information that leads people to make attributions, while the latter is a way of describing and categorising the various causes. For example, information concerning the behaviour of others (consensus) affects the selection of the cause of an event, whereas the selected cause can be categorised as an internal or external one. Weiner's (1986) attributional theory of 
motivation focuses on the causal dimension of the chosen cause and suggests that its causal dimension determines the subjective perception of future goal's attainment (expectancy shifts).

Weiner (1986) proposed that the perceived causes of events can be subsumed within three basic causal dimensions. One such dimension is the locus of causality; that is, whether the result of an action is perceived to depend on factors within the person or factors within the environment. The second causal dimension has been labelled stability and refers to a cause being perceived as stable and relatively enduring, or unstable and subjected to momentto-moment/periodic fluctuations. The third causal dimension is controllability and concerns the extent to which the cause is perceived to be under the control of the actor or anyone else.

Social learning theory has been criticised by Weiner (1986) on the grounds that by using the locus of control dimension to explain expectancy shifts in skill and chance tasks it failed to recognise that locus and controllability are two distinct dimensions of causality. A cause, therefore, might be internal, yet uncontrollable. For example, an individual might attribute his/her poor performance at basketball to the fact that he/she is short. Being short is an internal, but uncontrollable factor.

In addition, Weiner (1986) states that social leaming theory correctly proposes that expectancy shifts can be explained with causal constructs, but argues against the identification of the specific attributional dimension linked with expectancy shifts. Weiner's position is that the stability of a cause, rather than its locus of control, determines expectancy shifts. If the presence or absence of causes are expected to remain the same, then the outcomes experienced on past occasions will be expected to re-occur. A success under these circumstances would produce relatively large increments in the anticipation of future 
success and a failure would strengthen the belief that there will be subsequent failures. On the other hand, if the causal conditions are perceived as likely to change, then the present outcome may not be expected to repeat itself, or there may be uncertainty about subsequent outcomes. A success would yield relatively small increments, if any, and perhaps decrements in the expectancy of success, whereas a failure need not necessarily intensify the belief that there will be future failures.

The main finding of social learning theory that in skill related tasks expectancies tend to increase after success and decrease after failure is being explained in a different way by Weiner's attributional approach. In order to illustrate the different approach of social learning theory and attributional theory in interpreting typical shifts in skill tasks, the finding that success and failure in skill tasks are usually ascribed to ability will be utilised. Social learning theory suggests that ability is an internally controlled cause and that is why typical shifts are observed in skill tasks. On the other hand, according to Weiner's attributional theory, ability is thought to be a fixed property, such as visual acuity; therefore, because the causes of prior success or failure are perceived as relatively stable given skill-related tasks, future success should be anticipated with greater certainty in the case of prior success, while failure will decrease the levels of future expectancy to succeed. As far as chance tasks are concerned, social learning theory argues that success or failure tends to be ascribed to externally controlled factors (i.e., luck), while Weiner's attributional theory ascribes success or failure to unstable factors (i.e., a person is likely to reason that he/she had bad or good luck last time but that might not happen again). Expectancy, thus, should not rise and indeed could drop following a positive outcome or rise after a negative outcome either because of externally controlled or unstable attributions. 
Weiner argues that his attributional theory can explain the finding (i.e., Phares, 1957) that occasionally there are observations of atypical shifts in skill tasks and typical shifts in chance tasks, while social learning theory can not offer an explanation. Weiner's attributional theory suggests that there are instances that ability can be perceived as an unstable and changeable cause of an outcome, for example when ability refers to knowledge which is acquired through learning, and not to aptitude. In this case, atypical shifts in expectancy will be observed in skill settings. Regarding chance settings, one might conclude that he/she is a lucky or unlucky person (trait), or is riding a winning or losing streak. In these instances, the cause of the event is perceived as stable and that is why typical shifts are observed.

Finally, it should be clarified that Weiner's (1986) attributional theory of achievement motivation suggests that causal stability predicts shifts in the expectancy after success or failure, rather than actual expectancy of success or failure. Since many studies examining Weiner's attributional theory were conducted by using expectancy measures instead of expectancy change measures, Weiner (1983) suggested that the difference between expectancy and expectancy change is not crucial when the failure is objectively rather than subjectively defined. To illustrate the different effect of objectively and subjectively defined failure on expectancy an example provided by Weiner (1983) is presented. When subjects fail in solving a maths problem, a prediction derived from Weiner's attributional theory would be that, on the next maths problem, subjects perceiving their failure as due to aptitude (stable cause) will have lower expectancy of success than subjects ascribing their failure to bad luck (unstable cause). On the other hand, if failure is defined subjectively then a measurement of expectancy change should be used. Consider, for example, the investigation of the effect of subjectively defined failure in a test on expectancy of success. A bright pupil ascribed 
subjective failure (the grade B) to the difficulty of the exam (stable cause) and, therefore, anticipates the same grade when retaking an equivalent exam. A poor student attributes his/her subjective failure (the grade D) to bad luck or poor study strategy (unstable cause) and anticipates a better score when retaking an equivalent exam (grade C). In this case, if an expectancy measure is used the first subject anticipates a higher grade (grade B), even though his/her attribution of failure is stable, than the second subject who attributed failure to unstable causes. The attributional theory would be disconfirmed merely because the subjective levels of failure were different between the two subjects. This issue of different levels of subjective failure can be tackled by using an expectancy change measure which would indicate that the expectancy of success is not increased for the first subject (received grade B-expects grade B) because of attributing failure to a stable cause (the difficulty of the exam), while expectancy of success is increased for the second subject (received grade Dexpects grade $\mathrm{C}$ ) because of explaining failure in terms of an unstable cause (bad luck, or poor study strategy).

In conclusion, both social learning theory and Weiner's attributional theory suggest that the attributions people provide for the occurrence of an event affect expectancy for future success and subsequent levels of motivation. However, the two theories differ on the identification of the causal dimension that determines expectancy shifts and motivation; social learning theory suggests that the causal dimension of locus of control is the variable that has an effect on expectancy shifts and motivation, whereas Weiner's attributional theory identifies causal stability as the crucial variable. 


\subsubsection{Experimental comparison of the competing theories.}

A number of studies were conducted during the 1970 s and early 1980 s which aimed at a direct comparison between social learning and Weiner's attributional positions. The most important study was probably that conducted by Weiner, Nierenberg, and Goldstein (1976). Subjects were randomly assigned to one of six conditions $(0,1,2,3,4,5,6$ consecutive success experiences at the task) and were asked to indicate their expectancy of success prior to any task performance. To assess perceptions of causality, subjects were required to mark four rating scales that were identical with respect to either the stability or locus of control dimensional anchors, but which differed along the remaining dimension. For example, one attribution question was, "Did you succeed on this task because you are always good at these kinds of tasks or because you tried especially hard on this particular task?" "Always good" and "tried especially hard," the anchors on this scale are identical on the locus of control dimension (internal), but they differ in perceived stability, with "always good" a stable attribute and "tried especially hard" an unstable cause. In a similar manner, judgements were made between luck and tried especially hard (unstable causes differing in locus), these tasks are always easy and luck (external causes differing in stability), and always good and always easy (stable causes differing in locus). The results indicated that the increase in expectancy of future success was shown to be directly related to the perceived stability of a cause of the prior outcome (high stability is linked with higher expectation than is low stability). On the other hand, perceptions of the locus of causality were much more weakly (and insignificantly) related to stated expectancies of future success.

McMahan (1973) gave students five trials of repeated success or failure at an 
anagrams task. Prior to each anagram, subjects rated their confidence in reaching the correct solution (measure of expectancy of success). Following the trial and the announcement of the correct solution, subjects attributed causality for their success or failure by means of sets of six paired-comparison questions, all possible pairs of the ability, task difficulty, luck, and effort causal factors (i.e., I got the last word right mainly because: I tried hard or I was lucky). The results showed that in the failure condition effort (internal factor) was positively correlated with expectancies of success, whereas task difficulty (external factor) was negatively related to expectancies. These results provide a disconfirmation of Rotter's suggestion that locus of control affects the formation of subsequent expectancy. According to social learning theory, the correlation between effort (an internal factor) and expectancy is expected to be positive following success and negative following failure, while task attributions and expectancy are predicted to be negatively correlated after success and positively correlated after failure. The obtained correlations, however, were in the opposite direction. Moreover, these findings confirmed Weiner's theory since an unstable cause of failure (effort) was positively correlated with expectancy for future success and a stable cause (task difficulty) was negatively correlated with expectancy.

Kovenklioglu and Greenhaus (1978) classified college students' performance on two tests as a success or failure, if it met or failed to meet a minimum criterion of success that each student set prior to taking each test. Using a pair-comparison technique, students attributed their performance to ability, effort, task difficulty, and luck. The results showed that following failure the expected performance was positively correlated to low effort and negatively related to ability attributions. These results support Weiner's attributional theory, since firstly, low effort (unstable and internal factor) correlates positively with actual 
performance, and secondly, ability (stable and internal factor) is negatively related to levels of performance.

The comparison of the social learning theory and Weiner's attributional theory showed that the causal dimension of stability is a better predictor of future expectancies and levels of motivation than the dimension of locus of control. The study conducted by Weiner, Nierenberg, and Goldstein (1976) is particularly illustrative of the superiority of causal stability in the prediction of expectancies for future success. On the basis of theoretical grounds and this experimentally demonstrated superiority, Weiner's attributional theory seems to be a more successful paradigm for the investigation of the effect of attributions on motivation.

\subsubsection{The importance of the initial levels of motivation for the confirmation of Weiner's theory.}

Although the better predictive value of causal stability to locus of control was experimentally demonstrated, there are a number of studies that failed to confirm or contradicted Weiner's attributional theory.

Covington and Omelich (1979) demonstrated that in a failure situation none of the four major attributions (ability, task difficulty, luck, and effort) was shown to have a direct effect on subsequent performance, while effort was the only attribution that indirectly influenced performance through the intervening variable of future expectancy. However, the direction of the relationship between effort and expectancy was opposite to that predicted by Weiner's model. Rather than raising hopes for future success, low effort explanations for 
previous failure appeared to lower them. The interpretation suggested by Covington and Omelich was that the reason for such low effort may be critically important in determining future expectations, a factor that is not incorporated in Weiner's theory. For Covington and Omelich, a combination of low effort and pessimism for the future may reflect an underlying sense of despair that failure is a likely outcome no matter how hard one tries. In conclusion, the reasons for not trying initially may be far more important for understanding a subsequent motivational deficit than simply knowing that the individual did not try.

Weiner (1983) criticised Covington and Omelich's study for using a measure of expectancy rather than expectancy change, secondly, for using measures of motivation that were improper because of their inherent complexity and overdetermination by factors beyond the scope of his model, and thirdly, for misclassifying attributions on the dimension of causal stability.

In a later empirical examination of Weiner's critique, Covington and Omelich (1984) replicated their earlier test of Weiner's cognitive model using both their original expectancy variable (Covington \& Omelich, 1979) and an additional expectancy change variable. They also used their original motivational variable, which was a measure of performance, as well as task persistence suggested by Weiner to be an appropriate motivational variable for testing his attributional theory. In general, the same pattern of disconfirmation of cognitive predictions was demonstrated for both performance and task persistence, regardless of which expectancy measure was used. Attributing failure to low effort led both to lower future expectancies and to greater expectancy decrements. Moreover, low task persistence depended on effort attributions, but in an opposite direction from that predicted by Weiner's attributional theory. Rather than subsequent task persistence being enhanced by little effort 
at a first failure, persistence actually diminished following little initial effort. The only criticism that Covington and Omelich did not handle adequately was Weiner's claim that there was in their study a misclassification of attributions on the dimension of causal stability. Effort was a priori classified as an unstable cause and ability as a stable one, while people's perception of these causes might differ, as effort expenditure can occasionally be seen as a stable characteristic of an individual and ability as subjected to change and improvement.

In Graham's (1984) study children were induced to fail an achievement task and subsequent expectancies for success, causal attributions for failure, and persistence on a similar task were measured. The findings showed that causal stability measured by ability (stable cause) and effort (unstable cause), did not predict persistence on the similar task either directly or indirectly through expectancies of success. Graham suggested that particularly with regard to persistence on the similar task, a number of intervening cognitions might influence the relationship between attributions and persistence such as intrinsic interest in the task. Therefore, the relation between causal stability and levels of after-failure motivation might be moderated by the degree to which the task is personally appealing to the person.

Causal attributions for repeated success and failure of individuals differing in their achievement needs were examined in a study by Dalal and Sethi (1988). Subjects were selected as high and low need achievers and were given anagram tasks on which they either succeeded or failed in all three trials. The results showed that high need achievers had greater future expectancies that they would do better on a similar task than low need achievers. In addition, high need achievers perceived effort expenditure as influencing achievement outcomes. Such an effort-outcome covariation principle was not manifested by the low need 
achievers. Subjects high in need of achievement appeared to be viewing effort as a necessary precondition for success. This attributional bias resulted in high expectancy of future success and facilitated the continuation of goal striving following failure. Dalal and Sethi (1988) demonstrated that "the predictions of Weiner's theory found better support among high nAch subjects than low n-Ach subjects, particularly under the failure condition." Therefore, it might be the case that it is not only the degree to which a specific task is appealing to the person that moderates the effect of causal stability on motivation; also individual differences in achievement motivation might have such an effect, since in failure situations high need achievers provided more effort attributions and had greater success expectations than low need achievers.

Winefield, Tiggemann, and Winefield (1992) examined the effect of attributions made by young unemployed people concerning the loss of their job on various emotional reactions. Subjects attributed the fact that they are unemployed to lack of ability (stable cause), the situation (stable cause), lack of effort (unstable cause), or bad luck (unstable cause). The results showed that in contrast with Weiner's theory, causal stability did not predict a state of hopelessness leading to motivational deficits. The explanation that Winefield, Tiggemann, and Winefield (1992) gave for these findings was that the rated causes might be misclassified on the dimension of stability. For example, it is well known that although teenage unemployment rates are very high, such rates are much lower in older age groups, which justifies the classification of the "situation" as an unstable rather than stable cause. Another possible explanation that can be given to these findings is that causal stability failed to predict motivational deficits because the different level of each participant's motivation to find a job was not taken into account. It might be the case that attributions of failure to find a job have 
an effect on people who are really involved in finding a job, while less involved individuals do not get influenced by the stability of the attributions. In other words, after failing the perceived stability or instability of the attributions determines whether motivational deficits will be present provided that people are highly motivated and thus, fight for success.

The failure to confirm or the disconfirmation of Weiner's theory of achievement motivation by these studies may be caused by the omission of a variable which moderates the effect of causal stability on subsequent motivation after failure. The initial motivation (or intrinsic interest) to engage in the task might have an effect on causal stability's power to predict future motivation for similar tasks. If the task is interesting and challenging for the individual then it is more probable that he/she will search for the causes of the failure and that the perception of these causes as stable or unstable will affect subsequent motivation to engage in similar tasks. On the other hand, if the person is not interested in the task then the attributional process might not be initiated and even if it is, the stability of the causes will not determine future motivation. For example, imagine that someone is given a problem to solve which he/she finds boring, and after failing to find an answer is asked why he/she failed; a reason that might be given is that of not trying hard enough. Effort attributions are classified as unstable ones, and therefore attributional theory would predict the maintenance or increase of their subsequent motivation to solve a similar problem. However, even though the amount of effort expended is changeable, it is highly possible that they will not try harder in the future, merely because they are not interested.

In conclusion, the failure to confirm or the disconfirmation of Weiner's theory might be the result of ignoring that the initial level of motivation to engage in a specific task might have a moderating effect on the relationship between causal stability and after-failure 
motivation. In this thesis it is suggested that the causal dimension of stability is a good predictor of after-failure motivation provided that the initial level of motivation to engage in the task is high.

\subsection{ATTRIBUTIONAL STYLE AND MOTIVATION.}

The previous sections focused on the relationship between attributions concerning a specific event, namely situational attributions, and the level of motivation following the occurrence of this event. In the current section the concept of attributional style will be discussed, as well as its relation to the psychological construct of motivation.

Since attributional style was developed within learned helplessness theory (Abramson, Seligman, \& Teasdale, 1978) it is useful to start with a short presentation of the theory. According to learned helplessness theory, learning that outcomes are uncontrollable results in motivational deficits. As the individual comes to expect that outcomes are uncontrollable he/she exhibits helplessness and lack of motivation, which consists of retarded initiation of any voluntary response. The attribution people make for the occurrence of uncontrollable events, determines the chronicity and generality of their helplessness deficits. The chronicity of the deficits depends on whether the attribution is to stable or unstable factors; attribution to stable factors leads to chronic helplessness because they imply to the individual that he/she will lack the controlling response in the future, as well as now. If attribution is to an unstable factor, then he/she will not necessarily expect to be helpless in the future. The generality of the helplessness concerns whether the person generalises the expectation of non-contingency to a new, controllable situation by attributing helplessness to global causes (causes that are 
perceived as influencing performance in a greater variety of situations).

In their theory Abramson, Seligman, and Teasdale (1978) also discussed the concept of attributional style, which is a tendency to explain events in a habitual way. Attributional style is a result of accumulated past experience which leads to the development of specific attributional patterns that people use in order to explain various events in their lives. People tend to provide causes of their successes and failures that are similar as far as the causal dimensions of internality, stability, and globality are concerned, showing possible systematic biases and errors in the formulation of attributions. Abramson et al. (1978) primarily talked about a vulnerable attributional style defined as an inclination to attribute failures to internal, stable, and global factors and successes to external, unstable, and specific ones.

A vulnerable attributional style is related to low expectancy for future success that leads to motivational deficits and helplessness. People who tend to explain their failures by providing stable and global causes and their successes by unstable and specific ones show low levels of motivation. In addition, systematically attributing failure to internal factors and success to external ones impairs self-esteem.

However, it is important to consider a discrepancy between learned helplessness theory and the conceptualisation, as well as the measurement, of attributional style. Even though leamed helplessness theory suggests that motivational deficits are caused by the perception of a response-outcome non-contingency (uncontrollable event) and the attribution of this event to stable and global factors, the notion of uncontrollability has been eliminated from any operational measurement linked with the theory; that is, the measurement of attributional style (Weiner, 1991). More specifically, measures of attributional style consist of a number of positive (successes) and negative (failures) events which can be either 
controllable or uncontrollable. For example, the most widely used measure of attributional style, namely the Attributional Style Questionnaire (ASQ; Peterson, Semmel, Von Baeyer, Abramson, Metalsky, \& Seligman, 1982), contains outcomes such as "you do a project that is highly praised" or "you go out on a date and it goes badly" which are not necessarily perceived as uncontrollable events. Therefore, although learned helplessness theory suggests that stable and global attributions for uncontrollable negative events impair subsequent motivation, the huge research literature on attributional style disregards the controllability or uncontrollability of the events; the literature suggests that a tendency of attributing failures to internal, stable, and global factors, as well as successes to external, unstable, and specific ones, results in motivational deficits.

In conclusion, a vulnerable attributional style, that is, a systematic attributional bias of providing internal, stable, and global causes for failure and external, unstable, and specific ones for success leads to the impairment of motivation. People who show the above systematic biases are characterised by low levels of motivation as they tend to generalise negative outcomes across time and situations (Abramson, Seligman, \& Teasdale, 1978).

\subsubsection{Some considerations regarding the conceptualisation and measurement of attributional style.}

One main issue concerning the concept of attributional style is whether it shows acceptable levels of cross-situational consistency in order to be valid as an individual difference construct. That issue refers to the fundamental question "Does attributional style exist?" In an attempt to critically evaluate the concept of attributional style Cutrona, Russell, 
and Jones (1985) found that the best fitting model to their data hypothesised both crosssituational consistency and situational specificity. On the grounds of their findings they suggested that a refined theory of attributional style might encompass the notion of more narrowly specified classes of situations. In addition, Anderson, Jennings, and Arnoult (1988) argued against the prevailing view of attributional style as totally (or nearly totally) crosssituationally consistent. They proposed the conceptualisation of attributional style at a moderate level of specificity; that is, people tend to make similar attributions about the occurrence of events that can be classified into the same category. For example, healthrelated events or occupation-related events might comprise classes of situations that are defined at a moderate level of specificity.

The measurement of attributional style has shown relatively low levels of internal reliability, which mainly concerns the causal dimension of internality. In the relevant literature it has been suggested that a possible reason for the low levels of the reliability of the attributional style questionnaires is the small number of items that are used for the calculation of the internal reliabilities. In an attempt to tackle the problem of low internal reliabilities, Peterson and Villanova (1988) increased the items of the Attributional Style Questionnaire (Peterson, Semmel, Von Baeyer, Abramson, Metalsky, \& Seligman, 1982) from six (negative events) to twenty four (negative events). By increasing the number of items the Cronbach coefficients reached acceptable levels ( .66 for internality, .85 for stability, and .88 for globality).

However, the dimension of internality shows consistently the lower levels of internal reliability (Cutrona, Russell, \& Jones, 1985; Peterson \& Villanova, 1988; Bums \& Seligman. 1989; Furnham, Sadka, \& Brewin, 1992; Furnham, Brewin, \& O'Kelly, 1994; Heaven, 1994) 
which leads to scepticism whether this causal dimension should be a component of attributional style.

Two theoretical reasons can be provided for explaining why especially the causal dimension of internality shows low levels of internal reliability. Firstly, as respondents are asked to vividly imagine an event happening to them and to provide the major cause of this event (the ASQ format), many interrelated causes are retrieved as possible explanations. However, the respondent has to restrict himself/herself and give only one cause explaining the event. The fact that the respondents perceive a complex causal reality and at the same time have to provide only one cause leads them to give causes that in one event represent attributions about the self (internal) and in another event attributions about others or the circumstances (external). That is not the case with the stability or globality of the attributions because choosing a stable or unstable factor and a global or specific one is probably not perceived as determining whether an explanation is biased, self-centred, or even selfenhancing as can be an internal or external one. For this reason the activation of the complex causal reality does not decrease the internal consistency of causal stability and globality.

Secondly, according to Miller, Smith, and Uleman (1981) the distinction of internal/external causes can be regarded as reflecting a more fundamental causal dimension; that is, the chosen/not chosen dimension. The dimension of chosen/not chosen concerns the distinction between acts deliberately chosen by the actor, or events caused more deterministically. Consequently, the respondents might focus on providing attributions that are demonstrating consistently that outcomes are the results of their own action or are caused deterministically rather than internal or external.

Another point regarding the conceptualisation and measurement of attributional style 
concerns the accumulation of empirical data which are in contrast with the theoretical assumption that a vulnerable attributional style is the systematic attribution of negative outcomes to internal, stable, and global causes and the attribution of positive ones to external, unstable, and specific factors. It might be the case that it is not a single process that underlies attributions for positive and negative events, and that the attributions of the two types of events should not be considered as the polar opposites of the same dimension. Brewin and Shapiro (1984) have argued along the same line as far as the locus of control scales are concerned; that is, locus of control for positive outcomes should be regarded as distinct from locus of control for negative outcomes. In a number of studies (Ahrens \& Haaga, 1993; Corr \& Gray, 1994) internal, stable, and global attributions for negative events were either uncorrelated or negatively correlated with external, unstable, and specific attributions for positive ones. Therefore, Abramson, Seligman, and Teasdale's (1978) suggestion that a vulnerable attributional style is an inclination to attribute failure to internal, stable, and global causes and success to external, unstable, and specific ones, is questioned in this thesis.

In conclusion, the concept and measurement of attributional style has to be refined by firstly, using events that are contained in a psychologically meaningful category of situations, secondly, omitting the causal dimension of internality as it was repeatedly shown to have low levels of internal reliability, and finally, using negative and positive events separately. It is important that the refining of the concept and its measurement should be carried out before any attempt to investigate the joint effect of situational attributions and attributional style on motivation. 


\subsection{THE EFFECT OF SITUATIONAL ATTRIBUTIONS AND ATTRIBUTIONAL STYLE ON MOTIVATION.}

According to Weiner (1986), a theory of motivation should concentrate first on searching for general laws, rather than explore interactions between individual differences and the situational variables affecting motivation, mainly because of the difficulties of personality measurement. There has been an attempt to combine the general law concerning the influence of causal stability on motivation and individual differences in the level of achievement needs (Atkinson, 1964), but Weiner argues that the results of this attempt were not fruitful because of the measurement problems of the concept of achievement needs and more specifically the lack of situational specificity of behaviour. People are not equally motivated to achieve in all situations; for example, individuals may be highly motivated to achieve in the classroom, but not in sports.

In addition to the articulation of the general law concerning the effect of causal stability on future expectancies and motivation, a number of studies examined the joint effect of causal stability and individual differences in attributional style on motivation.

Anderson (1983) investigated the influence of experimentally manipulated situational attributions for failure and attributional style on subjects' subsequent success expectancies, motivation, and actual performance. Participants were preselected on the basis of their attributional styles forming two groups which contained either character-style attributers (individuals with the tendency to make stable attributions for negative events) or behaviouralstyle attributers (individuals with the tendency to make unstable attributions for negative events). Research participants in the two pre-selected groups (character-style vs. 
behavioural-style) were randomly assigned to one of three experimental manipulations of situational attributions for failure: (a) no manipulation, (b) ability/trait manipulation, and (c) strategy/effort manipulation. The ability/trait condition encouraged participants to attribute failure to stable causes, while the strategy/effort condition encouraged participants to attribute failure to unstable factors. The findings showed that in the condition of no experimental manipulation of attributions, attributional style had an effect on the composite index of success expectancies, motivation, and performance; that is, participants with a behavioural-style had higher success expectancies, higher motivation, and performed better than participants with a character-style. On the other hand, the effect of the attributional style on the composite index was eliminated when subjects were provided with situational attributions. Success expectancies, motivation, and actual performance were lower in the condition of ability/trait attribution compared to the condition of the strategy/effort attribution. Therefore, experimentally controlled attributions for a specific failure event had a more dominant effect on motivation than individual differences in attributional style.

Mikulincer (1990) conducted a study to assess the effect of situational information and attributional style on the choice of stable or unstable attributions. Situational information consisted of experimental instructions encouraging the subjects to attribute failure to stable or unstable factors, while attributional style was measured by a Hebrew version of the Attributional Style Questionnaire (Peterson et al., 1982) containing four failure events. The findings indicated that all subjects, irrespective of their attributional style, made more stable attributions when situational cues indicated a stable cause than when they indicated an unstable cause. Attributional style was a reliable antecedent of the particular stable or unstable attribution made by the subjects only when they were exposed to failure without any 
attribution-eliciting information. Mikulincer (1990) concluded that-as far as the dimension of causal stability is concerned-these findings imply that situational information was more dominant than attributional style.

The research findings indicate that situational information regarding attributions eliminates the effect of attributional style on people's motivation. Therefore, Weiner's causal stability as an attribution of a specific situation (event) seems to be a better predictor of success expectancies and motivation than a measurement of attributional style. However, the superiority of situational information over attributional style occurs when the situational information leading to the formulation of attributions is not ambiguous, as in the case of experimentally controlled situational information in which the researcher tries to provide subjects with indisputable causes of an outcome. This competing relationship between situational information and attributional style might not take place when people make attributions for an event by using ambiguous information. In this case the attributional style of the person might have an effect on the interpretation of the situational information, and both of these variables might lead to the selection of a specific cause or causes for explaining the event. It is, thus, interesting to investigate the effect of attributional style on the choice of stable or unstable attributions after failure, when there is absence of indisputable situational information.

In conclusion, the effect of situational attribution on motivation might not be more dominant than the effect of attributional style. In this thesis, it is suggested that in the case that situational information is ambiguous there is a joint effect of situational attributions and attributional style on the levels of motivation. That is, attributional style affects the way situational information is perceived which in turn determines motivation. 


\section{PART II.}

A reformulation and extension

of Amabile's social psychology of creativity 


\subsection{A CONTRIBUTION OF ATTRIBUTIONAL THEORY TO THE REFORMULATION OF AMABILE'S SOCIAL PSYCHOLOGY OF CREATIVITY.}

\subsubsection{Introduction.}

Part two concerns a critique and a reformulation of Amabile's (1983) model of the social psychology of creativity based on attributional theory. Amabile's model suggests that when the outcome of the creativity process is self-perceived failure then motivation for similar tasks should decrease. However, Weiner's attributional theory stresses the effect of attributions for failure on the levels of subsequent motivation suggesting that failure per se does not lead to lower motivation. Thus, it is proposed that Amabile's model has to be reformulated so that the moderating role of attributions in the relationship between failure and subsequent motivation is taken into account. The creativity process is the ideal framework for testing the moderating role of attributions for failure on subsequent motivation because creativity demands high motivation and persistence; therefore, in practical terms there is a higher possibility that the condition of high initial motivation which is required for causal stability to be a good predictor of after-failure motivation is fulfilled.

In addition, the event of failing in a creativity task was utilised in order to test the hypothesised effect of attributional style on the perception of situational attributions that was suggested in part one.

Finally, following Amabile, Goldfarb, and Brackfield's (1990) suggestion that apart 
from continuing the examination of social factors that inhibit creative behaviour future research should look for social facilitators of creativity, an extension of the social psychology of creativity is proposed. An attempt is made in this thesis to investigate whether the existence of social norms encouraging creativity within a group (a creative atmosphere) might have a positive influence on the level of motivation to engage in a creativity task.

\subsubsection{Amabile's model of the Social Psychology of Creativity.}

The conceptualisation of creativity adopted by Amabile (1983) is based on the assumptions that (a) it is at least theoretically possible for anyone with normal cognitive abilities to be creative to some degree in some domain of endeavour, (b) there can be degrees of creativity within a particular individual's work; a scientist, for example, can do more creative and less creative scientific work, and (c) although different individuals can be quite distinct in their potential for creative performance in a given domain, it does appear to be possible to increase creativity to some extent.

In an attempt to define creativity Amabile offered a conceptual definition which is based on the product of the creative process and is the following: A product or response will be judged as creative to the extent that it is both novel and appropriate, useful, correct or valuable response to the task at hand, and the task is heuristic rather than algorithmic. Algorithmic tasks are those for which the path to the solution is clear and straightforwardtasks for which an algorithm exists. By contrast, heuristic tasks are those not having a clear and readily identifiable path to solution, tasks for which algorithms must be developed. However, if an algorithm for task solution exists but the individual has no knowledge of it, 
the task can be considered heuristic for that individual.

Amabile's (1983) model of creativity shows the influence of three factors, namely domain-relevant skills, cognitive skills, and task motivation, on the process of creative thinking. The component of domain-relevant skills includes familiarity with, and factual knowledge of, the domain in question: facts, principles, opinions about various questions in the domain, knowledge of paradigms, performance "scripts" for solving problems in the domain, and aesthetic criteria. Cognitive skills include an ability to understand complexities and to break cognitive sets or exploring new cognitive pathways. It also includes general rules that can be of aid in approaching problems or tasks (knowledge of heuristics) and a work style conducive to creativity as an ability to concentrate effort for long periods of time or to abandon unproductive strategies. Finally, an intrinsically motivated state is conducive to creativity, while an extrinsically motivated state is detrimental.

In addition, the model addresses the issue of the elements of the creative process, which are the following: problem or task presentation, preparation, response generation, and outcome. The first stage of the creative process is the presentation of the problem or the task and it can be done either by an internal process within the individual or by an external source. Problem or task preparation involves gathering information and resources for solving the problem or accomplishing the task. Then, one or more ideas or products are produced during the stage of idea generation, which is followed by a checking and an evaluation of the generated ideas during the stage of the idea validation. Finally, the last stage of the creative process takes place, which is the actual outcome and has three possible levels: (a) complete attainment of the goal (success), (b) no reasonable response possibilities generated (failure), and (c) some progress toward goal. The outcome of one cycle of the creative process can 
directly influence task motivation, setting up a feedback cycle through which future engagement in the same or similar tasks can be affected. If complete success has been achieved there will be no motivation to undertake exactly the same task again because that task has been completed. However, with success, intrinsic motivation for similar tasks within the domain should increase. If complete failure has occurred, if no reasonable responses were generated, intrinsic motivation for the task should decrease. Finally, if partial success has been met, intrinsic motivation will either increase (when the person who tries to solve the problem gets warmer in approaching the goal) or decrease (when the outcome shows that the person is no closer to the solution). Amabile's model of creative thinking is presented in figure 2.1.

Within Amabile's model of creativity the effect of task motivation on creative thinking has been extensively studied. Task motivation is seen as the most important determinant of the difference between what a person can do and what he/she will do (Amabile, 1983). As far as task motivation is concerned, Amabile and her colleagues have articulated the intrinsic motivation principle of creativity which suggests that an intrinsically motivated state is conducive to creativity, whereas an extrinsically motivated state is detrimental. People are said to be intrinsically motivated to engage in a particular task if it matches their existing preferences and interests. The effect of intrinsic versus extrinsic motivational orientation on creative work was demonstrated by Amabile (1985) in a study concerning creative writing. Subjects already involved in writing were asked to compose a poem after ranking either intrinsic or extrinsic reasons for doing creative writing. The design also included a control group in which subjects were not given any reasons to consider. The results showed that the creativity of subjects in both the intrinsic condition and the control condition was fairly high, 
and comparable to the levels of creativity shown in initial poems written before the experimental intervention. On the other hand, the creativity of the subjects in the extrinsic condition was markedly lower. The findings showed that concentrating on extrinsic reasons for creative writing resulted in a temporary decrease in creativity.

Amabile's creativity model, in accordance with the "overjustification hypothesis" derived from the attribution theory of Bem (1972), proposes that there is an inverse relation between extrinsic constraints imposed on an individual's engagement in an activity and that individual's intrinsic motivation to perform that activity. The overjustification hypothesis suggests that when people do not have a clear and salient awareness of their intrinsic interest in the task and perform the task in order to meet some extrinsic contingency, they tend to infer that their task engagement was motivated only by the constraint and not by their own interest. For Amabile, Hennessey, and Grossman (1986), extrinsic constraints such as reward, evaluation, and competition for prizes undermine intrinsic motivation when the task is perceived as a means to the extrinsic end. For example, explicitly contracting to do an activity in order to receive a reward will have negative effects on creativity by lowering the levels of intrinsic task motivation to engage in the task. The general mechanism through which external constraints have a detrimental effect on creativity is intrinsic task motivation. External constraints lower the levels of intrinsic motivation, which leads to less creative thinking.

The term "social psychology of creativity," which is frequently used to describe Amabile's work in the area of creativity, concerns the examination of the detrimental effect of a number of social constraints, such as evaluation, surveillance, competition for prizes, reward, and restricted choice in task engagement, on the levels of creativity through the 
mechanism of intrinsic motivation (Amabile, 1979; Amabile, Goldfarb, \& Brackfield, 1990; Amabile, 1982; Amabile \& Gitomer, 1984). For Amabile (1988), these social factors are extrinsic constraints intended to control or seen as controlling the individual's performance on the task in a particular instance. As such, the constraint is extrinsic to the task; it is not an essential feature of task performance, but it is imposed by the social environment. A salient extrinsic constraint is one whose controlling implications are clear to the individual during task performance.

More recently, Amabile, Goldfarb, and Brackfield (1990) suggested that future research should also examine other varieties of social influence on creativity. Apart from continuing the search for social factors that inhibit creative behaviour, the circumstances under which social facilitation of creativity occurs should be identified. For example, most of the literature on creativity training proposes that work in groups is more conducive to creative productivity than solitary work. In group settings individuals may stimulate one another's thinking, leading to a larger number of unusual ideas.

2.1.3 An attributional critique and reformulation of Amabile's creativity model.

One aim of this thesis is to examine the mechanism of the feedback cycle described by Amabile; that is, the effect of a single outcome of the creativity process on subsequent task motivation and, more specifically the effect of failure, as an outcome of this process, on motivation. The model suggests that if failure has been encountered, intrinsic motivation for the task, as well as for similar tasks, should decrease.

However, Weiner $(1980,1986)$ has argued that a specific failure does not necessarily 
lead to lower levels of motivation to engage in similar tasks. What is important is the stability of the attributions that a person provides concerning this failure. If failure is attributed to stable causes, motivation for similar tasks will decrease; if failure is attributed to unstable causes, then motivation will stay at the same levels or might increase.

On the grounds of Weiner's attributional model of achievement motivation, a reformulation of Amabile's creativity model is put forward. When the outcome of the creative process is failure, the creativity model should incorporate the important factor of causal stability as a moderator of the effect of failure on subsequent motivation. Provided that the initial level of motivation to engage in the creativity task is high, failure leads to lower motivation if stable causes are used for explaining why this outcome occurred, while unstable attributions do not decrease and might even increase subsequent motivation.

At this point it has to be noticed that while Weiner's attributional theory concerns the moderating effect of attributions in the relationship between failure and motivation, Amabile's model refers to the effect of failure in a creativity task on a specific type of motivation which is intrinsic motivation. However, there is no possible reason to suggest that Weiner's theory is not applicable to intrinsic motivation as it is to motivation in general. Therefore, after-failure attributions are suggested to have a moderating effect on subsequent levels of intrinsic motivation.

Moreover, the effect of attributional style on the formulation of situational attributions is also examined. It is suggested that attributional style, as it is the result of accumulated past experiences leading to habitual patterns of attributions, effects the perception of situational information and the attributions provided for the occurrence of a specific event. More specifically, a pessimistic attributional style, which is the attribution of 
negative outcomes to stable and global factors, has an effect on the choice of attributions for a specific failure event. The pessimistic attributional style leads to a tendency to perceive situational information in such a way that stable attributions for failure are quite frequently provided.

In conclusion, an attributional critique of Amabile's creativity model is presented, as well as a suggestion for its reformulation. In the case of failing in a creativity task intrinsic motivation does not necessarily decrease, since the attributions for failure determine an individual's interest to engage in similar tasks. Amabile's model needs to be reformulated in order to incorporate the moderating effect of attributions for failure on intrinsic motivation. Moreover, in order to test for a network of interrelated factors that influence after-failure motivation, the influence of a pessimistic attributional style on the formulation of the situational attributions for failure is examined.

\subsection{AN EXTENSION OF AMABILE'S THEORY: PRO-CREATIVITY SOCIAL NORMS AS A FACILITATOR OF CREATIVE} BEHAVIOUR.

Amabile, Goldfarb, and Brackfield (1990) suggested that the social psychology of creativity should look for the circumstances under which social factors might enhance creative thinking. Amabile's model of creativity has mainly focused on the detrimental effect of social constraints on creativity through the mechanism of intrinsic motivation. Social constraints, such as evaluation, surveillance, and reward, cause the decrease of intrinsic motivation to perform a task and therefore, supposedly impair creativity. The intrinsic 
motivation principle for creativity states that intrinsic motivation is highly important for the performance of any creative behaviour and that social constraints lead to a considerable decrease of a person's genuine interest to engage in a task.

In order to be able to identify the social facilitators of creativity it is important to consider the explanation that Amabile provides regarding the reason why some social factors have a detrimental effect on creative behaviour. The main explanation of the negative influence of social constraints on creativity concerns the possible narrowing of attention to stimuli external to the task. Important aspects of creative thinking, such as the flexibility and spontaneity of performance, as well as a tendency for risk taking, are decreased when attention to the intrinsic properties of the task is reduced. Therefore, the person tends to pay less attention to the task at hand, while the salience of its ending becomes much more important.

In an attempt to identify social factors that do not distract an individual's attention from the task it is suggested that the construction of a creative atmosphere within a group through the existence of pro-creativity social norms might enhance creative behaviour. Social norms for creativity play a rather discrete role in the promotion of creative thinking because these norms are implicit in most functional groups. On the other hand, social constraints, like rewards, are presented in a more salient way as most of the time there is a kind of contract which specifies the conditions for the attainment of the reward.

Finally, pro-creativity social norms might enhance creative behaviour through the effect of social comparison on the process of making attributions for negative events. According to the social comparison literature, after a threatening event (i.e., failure) individuals tend to compare themselves with someone who is inferior, less advantaged 
(Wood, 1989; Levine \& Green, 1984), or at least is perceived to be equal in the dimension under evaluation or the surrounding dimensions. By using this strategy individuals seek to reduce distress and/or enhance self-esteem (Gibbons, 1986; Lemyre \& Smill, 1985). Therefore, the group member whose idea failed to be accepted (by a judge being perceived as fair) or successfully implemented looks for comparison targets who in the past went through the same experience and failed.

In the case of a group that places a high value on creativity, it is more probable that failures are encountered since group members are encouraged to put into practice new ideas and even to take the risk of failing. On the other hand, groups that do not value creativity expect their members to do things in the "old way," which leads to avoiding failure. Thus, in groups characterised by pro-creativity social norms members can compare their own failures with the failures of other group members, which leads to the perception of the causes of failure as changeable and unstable. In the case, however, of groups that discourage putting new ideas into practice, if members dare to suggest a new idea and this idea fails either to be accepted or to be successfully implemented, people tend to perceive the causes of failure as rather unchangeable and stable, because they can not compare their own failure to other members' failures. Thus, the perception of the causes of failure differs depending on the group norms leading to different levels of after-failure motivation.

In conclusion, pro-creativity social norms might be a social facilitator of creativity as they play a rather discrete role in promoting creative behaviour, and consequently do not reduce intrinsic motivation for the task. Furthermore, social norms that encourage group members to be creative might possibly be conducive to creativity through the effect of social comparison on making attributions. Groups that encourage creativity encounter failures 
which enable members to compare their failure to the failures of others and to attribute them to changeable causes. On the other hand, groups that discourage creative behaviour do not allow for failures to occur; thus social comparison cannot be used for the maintenance of an optimistic state which can be based on the fact that other members of the group who failed in the past, managed eventually to succeed.

\section{Part III: Summary of chapter two.}

Social learning theory has suggested that achievement motivation is influenced by the locus of control of attributions that people make regarding various outcomes. In failure situations internally controlled attributions lead to decrements in achievement motivation, while externally controlled attributions lead to increments in achievement motivation. Bernard Weiner $(1980,1986)$ argued that causal stability is the variable that affects subsequent motivation and not locus of control. When failure is encountered and the causes of this event are expected to be present in the future, motivation will decrease; if the causes of failure are not expected to be present, motivation will be maintained or will even increase. Empirical work has supported Weiner's general law of the effect of causal stability on subsequent motivation, while locus of control was proven to be a less good predictor of achievement motivation. However, a number of studies have provided findings that either did not confirm or contradicted Weiner's attributional theory of motivation. A hypothesis is formulated in this thesis that this inadequacy of Weiner's model is due to the fact that the variable of the initial level of motivation has been neglected. Finally, the relationship between causal stability (for a specific event) and attributional style is explored. According to the 
relevant literature, situational causal stability is a better predictor of future motivation than measures of attributional style when the situational information is indisputable. In this thesis it is argued that the effect of attributional style and situational attributions on future motivation should be examined jointly, mainly because when situational information is ambiguous attributional style effects the formulation of situational attributions.

Weiner's attributional theory of motivation and the concept of attributional style are utilised for the reformulation of Amabile's model of creativity. Amabile suggests that any outcome of a given run through the creativity process effects motivation to engage in similar tasks. Moreover, each specific event of failure leads to lower levels of subsequent motivation. On the grounds of Weiner's model this thesis argues that a single event of failure may not impair subsequent motivation and that the perceived causes of this failure should be taken into account for the prediction of future motivation. If the person is highly interested in the task and makes unstable attributions for failure, motivation should not drop and might even increase. Attributional style, as a product of past experiences of success and failure, can influence the attribution of failure to stable or unstable causes, as well as the levels of future motivation. Finally, an extension of Amabile's creativity model is suggested on the basis of the identification of social factors that can enhance motivation to be creative. The suggested reformulation and extension of Amabile's creativity theory is presented in figure 2.2. 


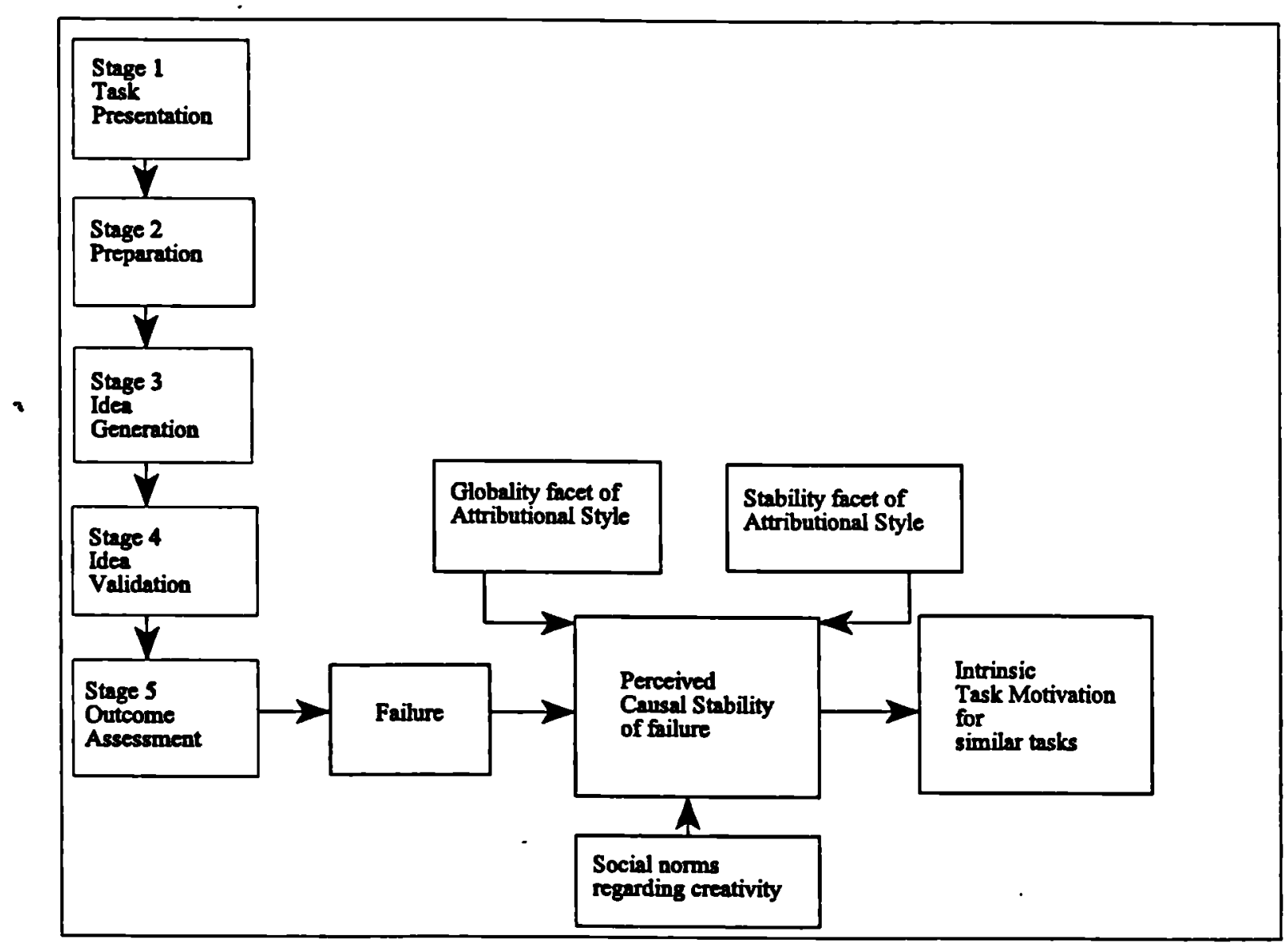

Figure 2.2. A proposition for the reformulation and extension of Amabile's creativity theory in the case of failure. 


\section{CHAPTER THREE}

\section{CREATIVITY/ INNOVATION AT THE WORKPLACE}

AND ORGANISATIONAL CULTURE 


\subsection{INTRODUCTION.}

The extension of Amabile's creativity model suggested in the first chapter, which involves the identification of social norms that facilitate motivation following failure in a creativity task, will be elaborated on further in the work setting. The relationship between organisational innovation and individual creativity at work will be clarified, as organisational innovation is closely related to the phenomenon of individual creativity. In addition, the concept of organisational culture will be introduced and utilised for the measurement of organisational social norms regarding creative behaviour, as well as other social norms of organisational culture/climate which are presented in the innovation literature as facilitators of creativity.

The extension of Amabile's model within organisational behaviour concerns the effect of organisational culture, as a set of social norms, on motivation to be creative at work. More specifically, when employees come up with a new idea for the improvement of the company's operation (ideation component of innovation) and this idea fails to be accepted for implementation, their subsequent motivation to engage in a similar task depends partly on the organisational norms that operate within their work group.

\subsection{ORGANISATIONAL INNOVATION.}

\subsubsection{The concepts of innovation and creativity.}

Zaltman, Duncan, and Holbek (1973) define innovation as "...any idea, practice, or 
material artifact perceived to be new by the relevant unit of adoption" (p. 10). Kanter (1983) suggests that innovation refers to the process of bringing any new, problem-solving idea into use. Ideas for reorganising, cutting costs, improving communication, or assembling products in teams are seen as examples of innovation. For Kanter, innovation is the generation, acceptance, and implementation of new ideas, processes, products, or services. Moreover, application and implementation are central to the definition of innovation; it involves the capacity to change or adapt. West and Farr (1990) define innovation "as the intentional introduction and application within a role, group or organisation of ideas, products or procedures, new to the relevant unit of adoption, designed to significantly benefit the individual, the group, organisation or wider society" (p. 9).

There are mainly three characteristics of innovation that seem to appear in the various definitions of innovation suggested in the literature. The first attribute of innovation is novelty which can be either absolute (something totally new) or relative (something new to a specific unit of adoption, but already well known and implemented). The second attribute is an application component which refers to innovation not only as the generation of ideas but also to their implementation, and the final aspect of innovation is intentionality of benefit which distinguishes innovation from serendipitous change.

Creativity has been defined by either focusing on the creative process or on the result of this process (the product of creativity). For example, Amabile (1983) defines creativity as the activity which leads to a novel, appropriate, and valuable product, while Torrance (1988) refers to creativity as the process of identifying problems, making hypotheses about the deficiencies and testing them.

According to West and Farr (1990), the distinction between innovation and creativity 
"is one of emphasis perhaps rather than category" as "creativity appears to be understood more as absolute novelty (bring into existence) rather than the relative novelty of innovation (bring in novelties)" (p. 10). Innovation may involve creativity at the ideation component of the innovative process, but not all innovations will be creative. For instance, setting up quality circles in a company for the first time can be characterised as an innovation; it is a new procedure to the unit of adoption, but it does not involve creativity as quality circles are already widespread.

Although West and Farr (1990) suggest that the ideation component of innovation involves creativity, Kirton's (1976) adaptive-innovative theory claims that innovation is unrelated to levels of creativity. To Kirton (1978), adaptation-innovation is a cognitive style which refers to a preference of how things should be done. The difference between adaptors and innovators is one of style rather than level of creativity; that is, adaptors and innovators can be equally creative. However, Kirton's theory has been questioned and empirical evidence has been provided showing that high innovation is associated with high creativity (Goldsmith \& Matherly, 1987).

West and Farr's (1990) definition of innovation also refers to intentionality of benefit. Such intentionality may not exist in the case of a poet who writes creatively without concentrating on the possible rewards that might follow. Finally, innovation is seen by West and Farr to be a social process with the elements of the process being events that occur among people, while creativity is an individual cognitive process in which events occur within the individual. Innovation has a clear social and applied component since it impacts directly or indirectly upon others effected by the role, or others in the work group, organisation or wider society. West and Farr conclude that this necessary applied social 
component perhaps most sharply distinguishes it from creativity.

The West and Farr labelling of innovation and creativity as "social and individual cognitive processes", respectively, could be misleading. As one of the attributes of innovation is the implementation of the new idea and as creativity is not necessarily associated with an implementation stage, innovation is more likely to have an effect on a wide range of people. However, it should be clarified that defining creativity as an individual cognitive process does not mean that creativity takes place within a person irrespective of any social-environmental influences. Amabile and her colleagues $(1982,1984,1986$, and 1990) have demonstrated the detrimental effects of external factors like evaluation, reward, and surveillance on individual creativity.

In conclusion, when innovation is characterised as an absolute rather than relative novelty, it involves creativity at the ideation component of the innovative process. Innovation and creativity are conceptually the same in the case where innovation refers to the generation of new ideas rather than the implementation of well-known ideas which are new to the relevant unit of adoption. Therefore, the concepts of innovation and creativity will be treated as interchangeable as far as the ideation component of innovation is concerned.

\subsubsection{Existing levels of analysis in the work innovation literature.}

Staw (1984) divides the innovation literature into three levels of analysis: individual, group, and organisational. This distinction is based on which of these, individual, group, or organisation, is the main unit of production or implementation of innovations. According to King (1990), at the individual level many writers continue to use the terms innovation and 
creativity interchangeably and much of the innovation literature draws upon the longestablished creativity research; at the group level there is by far less research conducted; and finally, the main focus of the majority of theoretical and empirical studies of innovation has been at the organisational level.

At the individual level of analysis research has focused on the traits associated with creativity, the situational influences, and the stages of the creativity/innovation process. In the mainstream literature of creativity an attempt to identify the personality traits that characterise the creative people has dominated. Some of the traits frequently held to be related to creative achievement are a tendency to be independent, high tolerance of ambiguity and complexity, propensity for risk-taking (MacKinnon, 1965; Child, 1973; Michael, 1979; Glassman, 1986). Research on situational influences on innovation/creativity have identified a number of factors that can have a conducive or detrimental effect on creativity. Moderate freedom of choice is cited as a positive antecedent of innovation (Bumside, 1990), as well as feedback and recognition from supervisors (West, 1989). As far as inhibitors are concerned, a highly centralised organisational structure with rigid vertical relationships is presented as detrimental to individual creativity (Kanter, 1983; Nystrom, 1979). Finally, research on creativity process has mainly attempted to provide some models considering the multiple stages of the creative process. In general terms, most of the models describing the creative process present the stages of task presentation, preparation, idea generation, and idea validation.

Even though there is research at the individual level of innovation/creativity that addresses the influence of social factors, in general such an effect on innovation has been neglected. The ideation component of innovation is primarily examined as an individual 
process which is taking place in a social vacuum. King (1990) suggests that a fuller understanding of innovation at the individual level demands that the person is always seen in his/her social environment. For King (1990), research which ignores social influences on individual innovations will inevitably be of limited applicability. There is a need to examine how social psychological processes have an effect on people's motivation to innovate.

Group innovation concerns the emergence, import, or imposition of new ideas which are implemented by the group through interpersonal discussions and negotiations leading to a possible reformulation of the original idea. Research on group innovation has mainly focused on the identification of those group characteristics which facilitate innovation. As far as group innovation concerns the production of new ideas, Amabile (1988) argues that the individual process and the small group process are similar. The most important reason for this is the difficulty to separate the individual idea contributions, as ideas begin in one form, then go through several stages of modification, addition, and amplification by group members which often occur in a brief period in time. Moreover, groups appear to go through the same process stages that individuals do. A problem is presented to the group and preparatory work has to be done; then members generate ideas both alone and together, work through these ideas and assess them.

At the organisational level of analysis there is research which concerns the investigation of the effect of organisational characteristics on innovation. The importance of organisational structure has been emphasised and the notion of the "innovation dilemma" has been introduced which refers to the possibility that the structural variables of formalisation, centralisation, and complexity have a detrimental effect on innovation at the early stages of the process (e.g., idea generation), but promote innovation at the implementation stage 
(Zaltman, Duncan, \& Hollbek, 1973). Recently, interest in the influence of another set of organisational variables on innovation has grown, which are organisational culture and climate. There are some attempts to identify the aspects of culture or climate that either promote or inhibit innovation/creativity.

Amabile (1988) argues that the three levels of analysis in innovation at work are closely interlocked. Individual and small group creativity are the most crucial elements in the process of organisational innovation. This is the case since the ideation component of organisational innovation depends on the generation of new ideas by the individual or the small group. On the other hand, features of the organisation can also be crucial determinants of individual and group creativity at any point in time.

\subsection{ORGANISATIONAL CULTURE.}

In order to test for the hypothesised effect of organisational social norms on employees' after-failure motivation to come up with new ideas for the improvement of the company's operation, the concept of organisational culture will be used. Organisational culture is defined as the social norms, values, and beliefs, that are commonly shared by the members of an organisation and underlies their activity. Since organisational social norms constitute one of the elements of organisational culture, they have been thoroughly examined within the organisational culture literature. Research on organisational culture has also developed a number of measures of organisational social norms and values. Moreover, the research literature on organisational innovation has investigated the effect of organisational culture on innovation. 


\subsubsection{Definition of Organisational Culture.}

The definitions of organisational culture refer to the way people think and act within an organisation defining culture in terms of both cognition and behaviour. Williams, Dodson, and Walters (1989) suggest that culture is the commonly held and relatively stable beliefs, attitudes, and values that exist within an organisation and underlies much of human activity in it. Degot (1987) speaks of the cultural element referring to the whole body of dominant perceptions that are shared by most of the employees of a corporation to function properly. It is pointed out that this system of shared perceptions tends to produce standard behaviour patterns and it is the result of the corporation's past experience as interpreted by those qualified to do so. Moreover, Margulies and Raia (1978) define culture as the commonly shared beliefs, values, and characteristic patterns of behaviour that exist within an organisation. According to Schwartz and Davis (1981), culture is a pattern of beliefs and expectations shared by the organisation's members that create norms which powerfully shape the behaviour of individuals and groups in the organisation. Finally, Deal and Kennedy (1982) have defined culture as "the way we do things around here" emphasising the issue of behaviour in their definition of culture.

In attempting to arrive at a definition other writers have also tried to formulate the "culture" concept. Hampden-Turner (1990) provides some information about the functions of culture in his definition of organisational culture. According to his theoretical perspective, culture comes from within people and is put together by them to reward the capacities that they have in common. It gives continuity and identity to the group and at the same time balances contrasting contributions. Furthermore, culture operates as a self-steering system 
which learns from feedback. It works as a pattern of information and can greatly facilitate the exchange of understanding. Culture's main function is to try to mediate dilemmas. The everyday issues arising within a corporation take the form of dilemmas: should new products be developed more quickly, to beat competitors on time; or more slowly, to win on quality? The larger strategic issues have the same characteristics; the organisation needs to preserve its key continuities, but it also needs periodic change. The whole area of corporate culture is constructed entirely of such dilemmas.

Another description of corporate culture, which highlights some of the functions of culture, is provided by Schein in his book "Organisational Culture and Leadership" published in 1985. Organisational culture is:

"a pattern of basic assumptions-invented, discovered, or developed by a given group as it learns to cope with its problems of external adaptation and internal integration-that has worked well enough to be considered valid and, therefore, to be taught to new members as the correct way to perceive, think and feel in relation to those problems" (p. 9).

It should also be mentioned that there are a number of organisational theorists who believe that corporate culture is actually the values of the top management. Lorsch (1985) takes culture to mean the shared beliefs of a company's top managers about how they should manage themselves and other employees. Gordon (1985) chooses to study culture through an upper-level group because he believes that the corporate values held by management are reflected in behaviour throughout an organisation. According to Martin (1985), in many organisations corporate cultures are developed from the philosophies of top management and maintained through the acceptance of these philosophies by the organisation's members.

Moreover, some researchers have suggested that different groups in the organisation develop their own particular culture, arguing against the assumption that organisational 
culture is always homogeneous. Gregory (1983) believes that many organisations are multicultural, composed of different occupational, divisional, and ethnic groups which approach organisational interactions with their own meanings and senses of priorities. Potter (1989) has criticised the way that the cultural concept has been formulated, arguing that it treats social groupings as far too homogeneous, and gives insufficient recognition to deviance, plurality, and change. Finally, Handy (1986), as well as Deal and Kennedy (1982), have pointed out that within any organisation a mix of different cultures can be found; different departments might possibly develop their own subcultures.

The definitions of organisational culture refer to the fact that the members of an organisation share the same values, beliefs, and attitudes, as well as social norms which dictate how one is expected to behave as a member of the organisation. These commonly shared cognitions and behavioural patterns are developed in order to give identity to the group, facilitate the confrontation of problems, and promote inter-group understanding.

\subsubsection{Elements of Organisational Culture.}

Organisational culture researchers in their attempt to formulate the concept of culture have tried to define the various elements of this conceptual construct. Schein $(1985,1990)$ suggests that organisational culture has three levels, which are the following: artifacts/creations, values, and basic assumptions. The three levels of culture interact with each other and there is a clear hierarchical structure based on the issue of awareness. The basic assumptions are considered to be unconscious, the values are characterised by a greater level of awareness, and finally, the artifacts/creations are visible. The artifacts and creations 
are the constructed environment of the organisation, its architecture, technology, office layout, manner of dress, visible or audible behaviour patterns, and public documents such as charts and stories. The second level of organisational culture consists of the organisation's values which are what "ought" to be done. For Schein, values are considered to be more difficult to identify compared to artifacts and creations. The final level is named "basic assumptions" and is the most important one in order to really understand a culture. Basic assumptions are invisible because they are taken for granted and organisational members are not aware of holding them. Therefore, they exist and act at an unconscious level which is their main difference to values, as values are assumed to be operating on a higher level of awareness. In fact, basic assumptions are values which begin to be taken for granted and gradually drop out of consciousness.

Martin and Siehl (1983) suggested that besides the three elements of culture described by Schein there is a fourth one; that is, management practices. This element contains the familiar management tasks, such as training, performance appraisal, allocation of rewards, hiring, and so forth. Management practices may or may not include artifacts. For example, a training program for new employees may be an occasion for telling organisational stories and may conclude with a ceremony. In general, artifacts and management practices express values, while underlying those values are even deeper assumptions, which rest at an unconscious level.

Even though a number of cultural elements are defined, researchers usually choose to study only one of these elements. Schein tries mainly to investigate and uncover the basic assumptions that are unconsciously leading the behaviour of the group members, while Martin and Siehl focus on the values as manifested in patterned sequences of events, rituals, 
and artifacts. Moreover, Cooke and Rousseau (1988) have studied another element of culture, namely the behaviours it takes to fit in and go ahead, which are evidence of the social norms attached to a group.

In attempting to incorporate all the elements of culture defined in the existing literature, Rousseau (1990) developed a model of the structure of organisational culture. The various elements are organised from readily accessible to difficult to assess (fig. 3.1). At the perimeter, material artifacts reflect the physical manifestations and products of cultural activity (e.g., logos, badges). Structures reflect those patterns of activity (i.e., decisionmaking, co-ordination, and communication mechanisms) that are observable to outsiders and whose functions help solve basic organisation problems. Social (behavioural) norms, as members beliefs regarding acceptable and unacceptable behaviour, are the next element of culture, while the fourth element is called "values" and reflects priorities or preferences for specific outcomes. Finally, fundamental assumptions comprise the centre of the cultural model and require researcher-member interaction in order to be assessed.

\subsubsection{Organisational culture and organisational climate.}

The conceptual overlap between organisational culture and climate is discussed in this section. This task is important since the literature on organisational innovation has discussed the effect of organisational culture, as well as that of organisational climate, on the process of innovation.

For Reichers and Schneider (1990), climate is widely defined as shared perceptions of organisational policies, practices, and procedures, both formal and informal. On the other 
hand, culture refers either to something the organisation is or something the organisation has. The first definition of culture promotes the study of culture within a native-view paradigm (emic approach), which focuses on asking respondents to make sense of their own behaviour. The second approach to culture, as something that the organisation has, applies an externalview paradigm (etic approach) in which the researcher provides the conceptual framework for the study of cultural perspectives. Reichers and Schneider (1990) argue that the second approach to culture (etic paradigm) is conceptually similar to the concept of climate. Within the etic paradigm, culture is defined as a set of shared meanings or understandings about the organisation and its problems, goals, and practices. Climate researchers have acknowledged the importance of shared perceptions (meanings) and have wrestled extensively with the operationalisation of the shared aspect of the perception. Reichers and Schneider (1990) suggest that there is a high degree of conceptual overlap between the two concepts, but culture can be distinguished from climate because it exists at a higher level of abstraction since some of its elements involve unconscious processes (e.g., basic assumptions). The concept of climate does not incorporate any aspect of unconscious processes, and it can be seen as a manifestation of the unconsciously held elements of culture.

Even though both organisational culture and climate emerged in order for organisational theorists to explain the effect of the social environment on human behaviour within the organisation, the two concepts have developed separately by each ignoring the theoretical advancements achieved by the other. One of the reasons for this isolation is the use of different research methods by the climate and the culture researchers. Climate research tends to be nomothetic by using quantitative methods, while research in culture is primarily idiographic, employing qualitative techniques to explain the various phenomena (Glick, 
1985). However, recently there has been an attempt by culture researchers to study organisational culture by the use of both qualitative and quantitative methods (Siehl \& Martin, 1988; Hofstede, Neuijen, Ohayn, \& Sanders, 1990). Therefore, climate can be conceptualised as an aspect of culture which is more accessible than other elements of the construct (for example, basic assumptions; Reichers and Schneider, 1990).

\subsection{THE RELATIONSHIP BETWEEN ORGANISATIONAL CULTURE/CLIMATE AND ORGANISATIONAL INNOVATION.}

3.4.1 The effect of bureaucratic aspects of organisational culture on innovation.

Harrison (1972) and Handy (1986) in an attempt to taxonomise organisational culture suggested that the degree of formalisation and centralisation determines in which category a corporate culture might fall into. A highly formalised and centrally directed organisation is characterised by a "role culture" (Harrison's term) or an "Apollo culture" (Handy's term), which are merely models of a bureaucratic corporate culture. Thus, in the organisational culture literature the variables of formalisation and centralisation, conceptualised as shared beliefs of the organisational members concerning the way the company is structured, have been used to describe a culture of bureaucracy. Even though these factors can be presented as objective attributes of an organisation's structure, they are also aspects of culture on the condition that they represent members shared perception of their organisation.

Nystrom (1979) in his book "Creativity and Innovation" distinguishes between innovative and positional companies (bureaucracies). Innovative companies are relatively 
unformalised and flexible, and should lead to greater organisational diversity by mixing together organisational members and widening their outlooks. On the other hand, positional companies are based on a bureaucratic model, which contains high formalisation, inflexibility, and low levels of organisational diversity. According to Nystrom, the favourable conditions for company creativity and innovation are: (a) the absence of formalisation, which refers to the rules and procedures for carrying out activities and the prescribed relationships between organisational members explicitly stated in various company documents, (b) flexibility and openness in combining and recombining information and resources through the elimination of various structural restrictions, and (c) organisational diversity; that is, to give the organisational members the opportunity to acquire a broad mixture of knowledge and experience within the company. Although Nystrom refers to organisational formalisation, flexibility, and diversity as structural or strategic properties of the organisation, in the organisational culture literature these variables are aspects of the corporate culture as far as they concern shared beliefs regarding the way the organisation is perceived by its members.

Kanter (1983) suggests that innovation is associated with a particular way of approaching problems which she calls "integrative"; that is, the willingness to combine ideas from unconnected sources. Innovative organisations are characterised by integrative cultures and structures which encourage the treatment of problems as "wholes," considering the wider implications of actions. Such organisations reduce rancorous conflict and isolation between organisational units; promote the exchange of information and new ideas across organisational boundaries and provide coherence and direction to the organisation. Even though there is a diversity of people in these organisations, mechanisms exist for transcending differences and a team-oriented co-operative culture flourishes. On the other hand, 
segmentalist (bureaucratic) cultures are anti-change oriented and prevent innovation. Segmentalist organisations are concerned with compartmentalising actions, events, and problems and keeping each piece isolated from the others. Problems are perceived as narrowly as possible, independently of their context or their connection to other problems. Companies with segmentalist cultures are likely to have segmented structures; a large number of compartments walled off from one another and only the minimum number of exchanges take place. The segmentalist culture impairs people's motivation to innovate because firstly, they are expected not to communicate with any other segment concerning possible problems, which limits the perception of a problem as a "whole", and secondly, they are expected to carry out specific tasks and to stay within the fences organisations erect between tasks.

Kanter (1983) argued that innovation can be promoted only if people can get the power they need to innovate. Organisational "power tools" consist of supplies of three basic areas which are information (e.g., data, technical knowledge), peer support (e.g., approval, legitimacy, backing), and resources (e.g., funds, materials, space, time). Three aspects of the operation and norms in action of innovating companies aid power circulation and access. Open communication systems help potential innovators locate information that can be used to shape and sell a project. Network-forming arrangements help them be in a position to build a coalition of supporters. Finally, decentralisation of resources helps them acquire the resources to mobilise for action.

In a case study of a medium size electronics company, Feldman (1988) investigated the influence of organisational culture, formed from and in reaction to the deeply held ideas of the founder, on attitudes towards and capacities for innovation. The innovation process involved the attempt of the founder of the company to set up a decentralised organisation by 
delegating responsibility for innovation to the divisions. Therefore, for Feldman (1988) decentralisation is an organisational characteristic that promotes innovation.

Aiken, Bacharach, and French (1980) examined the effect of organisational structures and perceived organisational processes on proposals for innovation by middle and lower echelon officials of administrative bureaucracies. Organisational structures refer to objective properties of the organisation which are size, horizontal and vertical differentiation, and role specialisation. Organisational processes refer to patterns of activity within the organisation as perceived by the members of the organisation, and include task, internal communication, contact with outside groups (e.g., clients, other organisations), and influence activities. Their findings indicate that the number of proposals made by middle and lower managers is influenced by different organisational structures and processes. Middle managers report a higher number of proposals for innovation when vertical differentiation and internal communication were increased. On the other hand, lower managers tend to make more proposals for innovation, firstly, when they perceive themselves to be more influential, and secondly, in the case that they report a more frequent contact with informational sources outside the organisation. Finally, even though Aiken, Bacharach, and French (1980) observed that the administrative bureaucracies were slow to introduce innovations, they also report that the initiation phase of change does take place in bureaucracies under certain circumstances. Therefore, bureaucratic aspects of organisational culture do not necessarily inhibit innovative behaviour.

In a meta-analysis of the relationship between organisational innovation and a number of its potential determinants, Damanpour (1991) found that formalisation and vertical differentiation are not related to innovation, while centralisation, functional differentiation, 
specialisation, internal and external communication are. Even though for formalisation, theory suggests a negative relationship, some researchers have emphasised the need for a well-established, rigid purpose and clearly specified work rules for the successful introduction of innovations. Zaltman et al. (1973) attempted to resolve the issue by proposing that low formalisation is needed for the first stages of the innovation process, ? while high formalisation facilitates the stage of implementation. A negative association was hypothesised between vertical differentiation and innovation, but a nonsignificant relation emerged. However, when the effect of vertical differentiation was examined on administrative and technical innovations separately, there was a positive association between vertical differentiation and administrative innovations and a nonsignificant relation between vertical differentiation and technical innovations. Finally, in accordance to the literature, centralisation was negatively related to innovation, whereas functional differentiation, specialisation, internal and external communication were positively correlated with innovative behaviour.

As far as the effect of bureaucratic aspects of culture on innovation is concerned, it is possible to conclude that high organisational formalisation has not been empirically associated with low levels of innovation; researchers suggest a different effect of formalisation on innovation depending on whether it concerns the initiation or the implementation stage of innovation. On the other hand, centralisation of power (the concentration of decision-making authority) has been shown to inhibit innovation as it decreases organisational members' awareness, commitment, and involvement. 


\subsubsection{The enhancement of innovation by a culture/climate that values creativity,}

achievement, and internal co-operation.

Cooke and Rousseau (1988) presented a model of the dimensions of organisational culture in which they incorporated 12 behavioural styles determining the way organisational members are expected to think and behave. The thinking and behavioural styles are shaped within each organisation by the demands of organisational roles and at the same time become characteristics of such roles because of the systematic selection of certain types of people. The 12 behavioural styles (or aspects of organisational culture) compose two general sets of styles, the constructive styles and the aggressive/passive styles. The constructive styles (selfactualization, achievement, affiliation, and humanistic-helpful) are oriented toward the fulfilment of the higher-order needs of social relatedness and self-actualization. The aggressive/passive styles (e.g., competitive, power-oriented, conventional, approval) involve the fulfilment of lower-level needs of security, such as the need to gain self-esteem through competition, the need not to be rejected. The main difference between the constructive styles and the aggressive/passive styles concerns the effect that they have on the motivation of employees to innovate and take risks. The constructive styles refer to the extent individuals are expected to overcome insecurity and innovate/take risks, while aggressive/passive styles indicate whether individuals are encouraged to focus on protecting themselves by either using aggressive or defensive methods (e.g., being oppositional or avoid conflict).

Cooke and Rousseau (1988) suggested that innovation is promoted by a culture that encourages self-actualization and achievement, as these values are related to creativity, risk taking and individual growth. The members of an innovative organisation and/or department 
are encouraged to develop themselves, set and accomplish their own goals, and take on new and interesting activities. Innovation is also enhanced by a culture of co-operation and humanistic orientation. Members are expected to be supportive, constructive, and open to influence in their dealings with one another. The organisation is characterised by a participative and person-centred style of management and a high priority is placed on constructive interpersonal relations. Therefore, innovation is facilitated by an organisational culture that combines a high value placed on individual creativity, achievement, and growth with a supportive and collaborative social environment.

Kanter (1983) proposed that a culture which values individual creativity and achievement motivation is conducive to innovation. According to Kanter (1983), innovation within the organisation can not flourish unless the organisation's norms favour change. Furthermore, a culture of pride based on high performance in the past, increases people's feelings of confidence in themselves and others leading to higher levels of achievement motivation. In this social environment employees are more likely to be motivated to set goals, take risks, and get positive feedback. Since change requires optimism and faith and both of these conditions are more plausible on a foundation of prior successes, companies with a successful past are more willing to take risks and promote change. Thus, knowing that innovation is mainstream rather than countercultural, coupled with pride-in-company, provides an incentive for initiative.

Kanter also emphasised the importance of co-operation and social support in order for people and organisations to be innovative. To ensure a culture of co-operation within the organisation, Kanter suggests that a system of mobility across jobs should be implemented, through which people can come to know the operations of neighbouring functions and also 
develop close relationships with a considerable number of co-workers. This acquisition of information, as well as social contacts, can promote the generation of new ideas since they enable employees to see new aspects of problems and actively ask for their peers' support to encounter these problems. Such a system of mobility across jobs has to be backed up by employment security to avoid the possible usage of defensive strategies to secure one's position, which is detrimental to change. Finally, a culture of co-operation is enhanced by the frequent use of integrative working-teams within or across departments.

Amabile (1988) argued that employees motivation to innovate can be influenced by two broad organisational components, which she named "organisational motivation to innovate" and "skills in innovation management." Organisational motivation to innovate refers to the basic orientation of the organisation to innovate and it is merely a value placed on innovation. An organisational climate where (a) innovation is perceived as important and (b) enthusiasm is expressed and support is provided for exploration of new ideas, is conducive to an individual's or group's motivation to be creative. Regarding skills in innovation management, Amabile incorporated in this organisational component a number of attributes of organisational structure, as well as organisational procedures and systems, that promote creative behaviour. An absence of both internal competition and frequent threatening evaluation accompanied with enthusiastic support and collaboration between groups and departments, access to power and resources, a participative and collaborative management style, and finally, open communication systems, are organisational characteristics that promote innovation. Within the organisational culture literature most of the organisational characteristics presented above are aspects of corporate culture, as far as they represent shared values of the organisational members. 
West and Anderson (1992) conducted a study of top management teams in hospitals in order to examine the group-level factors which facilitate or hinder innovation in working groups. They used Quinn's (1988) typology of organisational culture to describe and analyse enacted cultural beliefs and to examine the relationship between organisational beliefs and innovations. For Quinn (1988), there are four organisational cultures based upon two dimensions (internal vs. external orientation and flexibility vs. control orientation) which are the following: (a) group culture, (b) developmental culture, (c) hierarchical culture, and (d) rational culture. Group culture organisations are internal and flexible in orientation, emphasising the people within the organisation. Organisations with developmental culture are seen as emphasising innovation, creativity, growth, and dynamism in the provision of services. Organisations with hierarchical culture focus on order, the importance of rules/regulations, and are concerned with formality and stability. Finally, organisations with a rational culture place most emphasis on achievement, task accomplishment, production, and control of production processes. West and Anderson (1992) found that the developmental and group cultures are perceived by raters to be more innovative than the hierarchical and rational cultures. Furthermore, raters indicated that the innovations implemented by the developmental and group cultures are seen as more innovative (e.g., improvement of services) compared to the innovations implemented by the hierarchical and rational cultures.

In 1990 Nystrom presented a model of organisational innovation in which the variables of organisational culture and climate were introduced as important intervening factors affecting the outcome of innovation strategies. The themes of organisational culture that are presented in Nystrom's model to promote innovation are risk taking, and competitiveness with other companies, while over-concern with efficiency, profitability, and 
survival may be viewed as inhibiting innovation. Furthermore, Nystrom suggests a number of climate dimensions that were shown to be positively related to creativity and change such as challenge, idea support, achievement motivation, and risk taking.

Concerning the issue of the influence of competition on innovation, there are some theorists who suggest that internal competition promotes innovation. Peters and Waterman (1982) argue that intra-organisational competition leads to higher levels of innovation, while at the same time they describe the deconstructive effect of internal competition on innovation, as it entails high costs of duplication-cannibalisation, overlapping products, overlapping divisions, multiple development projects. Most of the researchers who suggest that there is a positive effect of competition on the innovative process refer to interorganisational rather than intra-organisational competition. Nystrom $(1979,1990)$ argues that active competition with other companies creates a greater need for innovation, if companies are to survive. According to Nystrom, even inter-organisational competition does not necessarily increase the rate of innovation for an individual company, since a company might not want to, or be able to, respond to a need for innovation imposed by the marketing environment.

In conclusion, a supportive culture that values individual creativity and achievement has been identified by a number of sources to be a facilitator of innovative behaviour. Organisations that encourage their members to take risks, not to be afraid of failure and to set their own goals are characterised as highly innovative environments. Moreover, innovation is promoted within a co-operative social environment in which team work flourishes and open communication is perceived as a necessary factor for individual and organisational growth. Competition can be a conducive factor to innovation mainly when it is 
manifested at an inter-organisational level (between companies), while internal competition can lead to an uncontrollable conflict within the organisation hindering members perception of the organisation as an entity with certain common goals.

\subsection{THE EXTENSION OF AMABILE'S CREATIVITY THEORY WITHIN ORGANISATIONAL BEHAVIOUR.}

This thesis suggests that when an employee proposes a new idea for the improvement of the company's operation which is not accepted as appropriate and valuable for implementation, the organisational norms have an indirect effect on the level of after-failure motivation to engage in similar tasks via the formulation of attributions for this failure and the expectancy of future success. An organisational culture which promotes creativity, achievement and co-operation has a positive indirect effect on after-failure motivation of organisational members to be creative through the formulation of unstable attributions for failure and high expectancy of future success. On the other hand, an organisational culture characterised by internal competition and centralisation of power has a negative indirect effect on after-failure motivation through the formulation of stable attributions for failure and low expectancy of success.

Organisational norms of creativity, co-operation, and achievement characterise innovative groups which promote risk taking; on the other hand, norms of centralisation of power and competition characterise non-innovative groups which emphasise the importance of avoiding failure. Therefore, groups encouraging creativity, co-operation, and achievement encounter more failures, as well as successes, compared to the groups which are power- 
oriented and competitive simply because firstly, their members are not afraid of failure and secondly, success involves taking risks. Experiencing failures makes possible for the members of the innovative groups to compare their failures to the ones of other members (social comparison) leading to unstable attributions for failure, while experiencing successes enhance beliefs of collective efficacy (Lawson \& Ventriss, 1992) which are related to high expectancy of success following a failure event.

The extension of Amabile's theory of social creativity is put forward on the grounds of the identification of organisational norms that facilitate creativity through the mechanism of intrinsic motivation. More specifically, it is suggested that an organisational culture which promotes creativity, internal co-operation, and achievement has a conducive effect on employees motivation to be creative, particularly after set-backs.

\subsection{SUMMARY OF CHAPTER THREE.}

In chapter one the extension of Amabile's theory of creativity was proposed which is an attempt to identify social facilitators of creative behaviour. The extension will be further elaborated in the context of creative behaviour within the organisational setting by investigating the effect of organisational norms on motivation to be creative at work. The concept of organisational culture, which is defined by Cooke and Rousseau (1988) as a set of organisational social norms, is utilised for the conceptualisation and measurement of norms within the organisation.

Organisational innovation and individual creativity are closely related mainly because innovation involves creativity at the ideation component of the innovative process. In the 
work innovation literature the individual level of creativity as a crucial factor of innovative behaviour in organisations has been examined. In addition to the individual level of analysis, the innovation literature incorporates the group and the organisational level of analysis. The group level concerns the identification of those group characteristics which facilitate innovation. Finally, the organisational level refers to features of the organisation that can be crucial determinants of individual and group creativity.

Organisational culture defined as the organisational members shared perception of the social norms concerning expected and accepted behaviour, has been proposed to affect organisational innovation. An organisational culture that encourages creativity and places a high value on individual achievement in a co-operative environment has been suggested to be the social environment that enhances innovative behaviour. On the other hand, a culture characterised by centralisation of power and internal competition has a detrimental effect on organisational innovation. Therefore, this thesis proposes that when employees have a new idea for the improvement of the company's operation which fails to be accepted for implementation, their motivation to engage in a similar task depends on the norms of the organisation. 


\subsection{ARTICULATION OF THE HYPOTHESES OF THIS THESIS.}

This thesis, with reference to different attributional models, has the following hypotheses:

Hypothesis 1. Weiner's causal stability of failure is a better predictor of after-failure motivation than social learning theory's dimension of locus of control, provided that the initial level of motivation to engage in the task is high.

With reference to the reformulation and extension of Amabile's creativity theory

Hypothesis 2.1. Failure in a creativity task per se does not decrease subsequent motivation to engage in similar tasks.

Hypothesis 2.2. Provided that the initial level of motivation to engage in a creativity task is high, stable attributions for failure lead to low levels of subsequent motivation, while unstable attributions for failure lead to high levels of subsequent motivation.

Hypothesis 2.3. Low levels of pessimistic attributional style lead to the formulation of unstable attributions for failure, while high levels of pessimistic attributional style lead to the formulation of stable attributions for failure.

Hypothesis 2.4. Pro-creativity social norms lead to unstable attributions for failure, whereas anti-creativity social norms lead to stable attributions for failure. 
With reference to the extension of Amabile's theory within organisational behaviour

In the case of failing in a creativity task at work:

Hypothesis 3.1. Given that the initial motivation to engage in the task is high, unstable attributions for failure lead to high levels of after-failure motivation, while stable attributions for failure lead to low levels of after-failure motivation.

Hypothesis 3.2. High expectancy of future success leads to high levels of subsequent motivation, while low expectancy of future success leads to low levels of subsequent motivation.

Hypothesis 3.3. Given that the initial motivation to engage in the task is high, organisational norms promoting creativity, achievement, and cooperation lead to unstable attributions for failure.

Hypothesis 3.4. Given that the initial motivation to engage in the task is high, organisational norms promoting centralisation of power and internal competition lead to stable attributions for failure.

Hypothesis 3.5. Organisational norms promoting creativity, achievement, and cooperation lead to high expectancy of future success.

Hypothesis 3.6. Organisational norms promoting centralisation of power and internal competition lead to low expectancy of future success. 


\section{CHAPTER FOUR}

SOCIAL LEARNING VS. WEINER'S ATTRIBUTIONAL INTERPRETATIONS OF MOTIVATION:

THE IMPORTANCE OF THE INITIAL LEVEL OF MOTIVATION 


\section{INTRODUCTION}

Social learning theory (Rotter, 1966) and Weiner's (1986) attributional theory make contrasting predictions regarding the influence of perceived causal factors on the expectancy of success and subsequent motivation following an event of success or failure. Social learning theory specifies that expectancy and future motivation are influenced by the locus of control of causal factors. More specifically, internally controlled attributions cause increments or decrements of expectancy and motivation after success and failure, respectively (typical shifts), whereas externally controlled attributions lead to lower expectations and motivation after success and higher expectations and motivation after failure (atypical shifts). On the other hand, Weiner's attributional theory specifies that expectancy and motivation are influenced by the stability of causal factors. If the perceived cause is expected to re-occur (stable cause) then success will be followed by an increase of success expectancy and motivation, while failure will be detrimental to both of these variables. If the cause is perceived as temporary and is not likely to re-occur, then success will lead to lower expectancies and motivation, whereas failure will not decrease expectancies for future success or subsequent motivation.

Even though the predictions of the two competing theories concern not only expectancy shifts after the attainment or nonattainment of a goal, but also subsequent levels of motivation, the bulk of studies (Weiner, Nierenberg, \& Goldstein, 1976; McMahan, 1973; Fontain, 1974; Meyer, 1973) comparing the two theories investigated the relationship between causal factors and expectancy shifts. Thus, previous research has focused on the indirect effect of causal factors on motivation via expectancy shifts neglecting the possible direct effect of attributions on future motivation.

Social learning theory is quite explicit concerning the determinants of the strength of 
motivation. If a person perceives a reinforcement as contingent upon his/her own behaviour (internally controlled), then the occurrence of a positive or negative reinforcement will strengthen or weaken potential for that behaviour to re-occur. If the reinforcement is perceived as being outside his/her own control (not internally controlled), then the preceding behaviour is less likely to be strengthened or weakened.

Even though Weiner's attributional theory has examined less extensively the influence of causal stability on subsequent levels of motivation than its impact on future expectancies and affect, Weiner (1980) maintained that attributions can be expected to have a direct effect on subsequent motivation for similar tasks. In general, Weiner's attributional model predicts that stable attributions lower subsequent motivation, while unstable attributions are associated with the same or higher levels of subsequent motivation. Most of the empirical research on Weiner's attributional model has focused on the influence of attributions on motivation through the mediating effect of expectancy, while the possible direct effect of attributions on motivation has been rather de-emphasised.

The aim of the current study is a comparison between the two theories concerning their predictions about the effect of attributions on subsequent motivation in the case that failure has been encountered in a creativity task. It is suggested that causal stability is a better predictor of the levels of motivation that follow an incident of failure than locus of control, provided that the initial level of motivation to engage in the task is high.

In this study it is argued that the internality of attributions for failure does not determine the levels of subsequent motivation. This is the case because when failure is attributed internally, it is not necessarily expected to re-occur. For example, if a person fails to solve a problem and attributes this failure to being tired, the motivation to solve a similar problem in the future can be high, since the cause of failure and, therefore failure itself, might 
not re-occur.

Furthermore, it is suggested that for Weiner's attributional theory to be confirmed as far as failure is concerned, the variable of the initial level of motivation should be incorporated in Weiner's attributional model. The fact that a number of studies failed to confirm or contradicted Weiner's theory of achievement motivation can be explained by the negligence of the influence of an important intervening variable, namely, the initial level of motivation, on the predictive power of causal stability. The initial level of motivation concerns the initial interest that the person shows in the task. For example, a person could be engaged in a problem solving task that is either appealing or not appealing to him/her in the first place, before any feedback indicating success or failure has been perceived by the person. If the person is genuinely interested in solving the problem and he/she fails to find an adequate solution, then an attributional process is likely to be initiated and the causal stability of the provided cause of failure is expected to affect subsequent motivation. On the other hand, if the person thinks that the task is not interesting and fails to come up with an appropriate solution, then the attributional process might not take place at all, and if it does, the stability of the cause of failure might not predict future motivation.

To illustrate the reason why causal stability will influence motivation only in the case of initially motivated individuals, consider the effect of a stable and an unstable cause of failing to solve a problem, namely, "not being clever enough" (stable cause) and "not tried hard enough" (unstable cause), under the two different levels of initial motivation. When the task is appealing to the person and he/she attributes failure to "not being clever enough" the expectancy of future success will decrease and he/she will be less motivated to engage in a similar task; if the interested person attributes failure to "not tried hard enough," next time he/she will try harder probably because the task is something challenging and interesting and 
the cause of failure is not expected to re-occur (unstable). On the other hand, in the case that the person is bored by the task the stable attribution (not clever enough) might not decrease expectations or motivation because they were very low in the first place (boring task that I can not accomplish); if the bored person attributes failure to "not tried hard enough" (unstable cause) future motivation will not increase because even though potentially the level of effort is changeable the person has no reason (motivation) to try harder in the future.

The results of a number of studies that disconfirmed or failed to confirm attributional theory of achievement motivation in a situation of failure may be explained by the fact that the initial level of motivation was not taken into account. Three studies are presented which either disconfirmed or failed to confirm Weiner's theory of achievement motivation. It is argued that Weiner's predictions are confirmed only if there is a high level of initial motivation.

Covington and Omelich (1984) induced subjects to attribute failure to one of the four major causes (ability, task difficulty, effort, and luck) and tested the effect of the attributions on expectancy change, and on two motivational variables; that is, task persistence and a measure of performance. The results showed that only attributions of effort influenced expectancy shifts and task persistence. The relationship, however, between effort and expectancy/motivation was in an opposite direction from that predicted by Weiner's attributional model. Effort attributions led to lower expectancies for success and decreased subsequent motivation (persistence in a similar task). Covington and Omelich suggested that even though effort is classified within attributional theory as an unstable factor, it causes decrements to subsequent motivation because it possibly indicates the reasons for not trying in the first place. They further argued that a combination of low effort and pessimism for the future may reflect a sense of despair that failure will take place no matter how hard one tries. Graham (1984) studied whether stable (ability) or unstable (effort) attributions made 
by a sample of children who failed in an achievement task, had an effect on expectations for future success and persistence in a similar task. The results showed that causal stability did not influence either expectancies of success or subsequent motivation. Graham argued that particularly with regard to the direct effect of causal stability on persistence in the similar task, the intrinsic interest in the task influenced the relationship between attributions and subsequent motivation. In other words, she suggested that the degree of the intrinsic interest in the task moderated the effect of the stability of the cause on future motivation.

In a study of the causes of unemployment, Winefield, Tiggemann, and Winefield (1992) asked the participants to attribute the fact that they were unemployed to lack of ability (stable cause), the situation (stable cause), lack of effort (unstable cause), or bad luck (unstable cause). It was found that causal stability did not predict a state of hopelessness. Winefield, Tiggemann, and Winefield interpreted these findings as a result of the misclassification of causes on the dimension of causal stability. For instance, they suggested that the classification of the "situation" as a stable cause might be wrong because it is well known that although teenage unemployment rates are high, such rates are lower in older age groups. Another interpretation that can be given to these findings is that causal stability did not predict hopelessness because the initial level of motivation to find a job was not taken into account. It might be the case that whether the causes are stable or unstable is important only when the person is highly motivated to find a job and, therefore, fights for success.

Convington and Omelich (1984) disconfirmed Weiner's theory by showing that an unstable attribution of failure leads to lower expectancies and motivation, while Graham (1984) and Winefield, Tiggemann, and Winefield (1992) findings indicated that causal stability is not a predictor of expectancies or motivation. Convington and Omelich suggested that for the interpretation of their results it might be important to look for the reasons that in the first 
place led to low motivation and Graham proposed that the levels of the intrinsic motivation in the task might moderate the effect of attributions on motivation. In this study it is argued that the initial level of motivation is the primary variable that determines whether causal stability predicts or not subsequent motivation when failure has been encountered. Causal stability is a good predictor of subsequent motivation given that the person is highly motivated to engage in the task at hand.

The hypothesis of this study is that causal stability predicts motivation following failure provided that the initial level of motivation to engage in the task is high, while locus of control does not; this is the case because the re-occurrence of the causes of failure rather than them being internally or externally located, determines the levels of after-failure motivation.

\section{METHOD}

Respondents. Respondents were 113 undergraduates at University College London. Half of them (57) were excluded from any analysis because they succeeded in solving the achievement task. Concerning the respondents who failed to provide an adequate solution to the problem (56), $9(17 \%)$ were men and $45(83 \%)$ women. $38(70 \%)$ of the respondents were between 17 and 20 years old, $12(22 \%)$ were between 21 and 30 years old, and $4(8 \%)$ were over their thirties. They participated as part of a course requirement in introductory psychology.

Achievement task. In order to obtain variations in the perceived reasons for failure and to set a realistic frame for the spontaneous attribution of failure, respondents were asked to solve a problem without being induced to fail. However, because this study was concerned 
with motivation following failure in a creativity task, only respondents who failed to come up with an adequate solution were included in any analysis.

The task that was used was Duncker's (1945) "radiation problem," which in this study was stated as follows:

Suppose you are a doctor faced with a patient who has a malignant tumour in his stomach. It is impossible to operate on the patient, but unless the tumour is destroyed the patient will die. There is a kind of ray that can be used to destroy the tumour. If the rays reach the tumour all at once at a sufficiently high intensity, the tumour will be destroyed. Unfortunately, at this intensity the healthy tissue that the rays pass through on the way to the tumour will also be destroyed. At lower intensities the rays are harmless to healthy tissue, but they will not affect the tumour either. What type of procedure might be used to destroy the tumour with the rays, and at the same time avoid destroying the healthy tissue?

The respondents were given as much time as they wanted to solve the problem and then, were presented with two adequate solutions which were: (a) reduce the intensity of rays on the way to the tumour by applying many low intensity rays from different directions simultaneously, so that the healthy tissue is not damaged and the tumour is destroyed, and (b) avoid contact between rays and healthy tissue by sending high intensity rays through an open route (e.g., oesophagus) via a tube. However, they were told that there could be other adequate solutions to the problem apart from the ones presented.

The radiation problem requires creative thinking mainly because it is an ill-defined problem and part of the task is to formulate the problem itself (Newell, Shaw, \& Simon, 1963). Furthermore, in line with the widely accepted view of Mednick (1962) that creativity involves the formulation of "remote associates" (forming cognitive associations between two elements that are not typically associated) the respondents were provided with a "remote associate"; that is, a story which could help them to solve the problem. That story is called the "parade story" and was presented as follows: 
A small country was controlled by a dictator. The dictator ruled the country from a strong fortress. The tortress was situated in the middle of the country, surrounded by farms and villages. Many roads radiatcd outward from the fortress like spokes on a wheel. To celebrate the anniversary of his rise to power, the dictator ordered his general to conduct a full-scale military parade. On the morning of the anniversary, the general's troops were gathered at the head of one of the roads leading to the forress, ready to march. However, a lieutenant brought the general a disturbing report. The dictator was demanding that this parade had to be more impressive than any previous parade. He wanted his army to be seen and heard at the same time in every region of the country. Furthermore, the dictator was threatening that if the parade was not sufficiently impressive he was going to strip the general of his medals and reduce him to the rank of private. But it seemed impossible to have a parade that could be seen throughout the whole country.

The general, however, knew just what to do. He divided his army up into small groups and dispatched each group to the head of a different road. When all was ready he gave the signal, and each group marched down a different road. Each group continued down its road to the fortress, so that the entire army finally arrived together at the fortress at the same time. In this way, the general was able to have the parade seen and heard through the entire country at once, and thus please the dictator.

This story helps toward the identification of the first solution presented above. The general divided his army into small groups, each group marched down a different road, and finally, the whole army arrived at the fortress simultaneously. In the same way, rays of low intensity are applied from different directions reaching the tumour simultaneously.

Questionnaires. The respondents were asked to fill in a questionnaire measuring intrinsic motivation to solve the radiation problem, causal stability and locus of control of their failure to solve the problem, and after-failure intrinsic motivation for similar tasks. Measures of intrinsic motivation were preferred to measures of general motivation because, in accordance with the creativity literature, intrinsic motivation rather than extrinsic motivation is influential on creative thinking. 
Intrinsic motivation to solve the radiation problem. Intrinsic motivation is assessed within the creativity research by asking the respondents to indicate the degree to which the task is interesting, challenging, and enjoyable (for example, see Amabile, 1990). As failure leads to a negative affect because of its nature (Weiner, 1986), any items that are used in the creativity literature to assess the enjoyment that the person gets out of doing the task were not included. Therefore, the measurement of intrinsic motivation concerned how much interesting and challenging the respondents perceived the problem to be (two items). The coefficient of internal reliability of this scale was .67 .

Causal stability and locus of control. Respondents were asked to write down the major cause of their failure to solve the radiation problem. In order to tackle the methodological problem of the misclassification of causes on the dimension of causal stability, which is apparent in many studies, the respondents were asked to give their own ratings concerning the stability and locus of the cause. The Revised Causal Dimension Scale's (McAuley, Duncan, and Russell, 1992; CDSII) subscales of stability and personal control were used, which have acceptable levels of internal consistency of .67 and .79 , respectively. McAuley, Duncan, and Russell (1992) demonstrated the construct validity of the entire inventory by conducting a confirmatory factor analysis. The coefficients of internal reliability for the subscales of stability and personal control obtained in the current study were .76 and .78 , respectively.

After-failure intrinsic motivation for similar tasks. After-failure intrinsic motivation was measured after the respondents had decided that they succeeded or failed to give an adequate solution to the radiation problem. The respondents answered two items concerning whether they perceived solving another problem of this typeto be an interesting and challenging 
task. The internal coefficient for this scale was .94 .

Procedure. The respondents were given the parade story, as well as two other stories which did not have any hints concerning how to solve the radiation problem, and were asked to read carefully one at a time and write a brief summary for each of them. The two other stories were used to make the connection between the parade story and the solution to the radiation problem more difficult to trace. When they had finished with the three summaries, the radiation problem was presented and the respondents were encouraged not to feel inhibited about suggesting any solution, and to avoid asking the opinion of the people sitting next to them as it was a procedure of individual and not group problem solving.

The respondents had as much time as they wanted to come up with an adequate solution to the problem. After they had finished with trying to solve the problem, they were asked whether they already knew the radiation problem (no one knew the problem) and whether they thought that the task was interesting and challenging. Then, they were presented with the two adequate solutions and had to indicate whether they believed that they succeeded or failed to find an appropriate solution. It was said repeatedly that there could be other adequate solutions to the problem apart from the two presented, and that it was up to them to decide if they succeeded or failed.

Respondents who succeeded were asked to provide the major cause of their success and respondents who failed were asked to write down the major cause of their failure. Then, these causes were rated by the respondents on the subscales of stability and personal control. Finally, intrinsic task motivation for similar tasks was measured.

Following the questionnaires a complete explanation of the study was given to the respondents. 


\section{RESULTS}

A number of regression analyses were conducted in order to test the hypothesis of this study. Table 4.1 presents descriptive statistics of the measures of locus of control, causal stability, and after-failure motivation.

Table 4.1. Descriptive statistics of locus of control (LC), causal stability (CS) and after-failure motivation(AFM) for the whole sample, the subgroup of high initial motivation (SHIM) and the subgroup of low initial motivation (SLIM).

\begin{tabular}{lclllll}
\hline & Whole Sample & & SHIM & & SLIM & \\
& M & SD & M & SD & M & SD \\
LC & 14.77 & 3.93 & 15.09 & 3.81 & 13.21 & 4.35 \\
CS & 9.87 & 3.90 & 9.65 & 3.73 & 10.44 & 4.30 \\
AFM & 8.51 & 3.93 & 10.44 & 2.69 & 4.14 & 2.80 \\
& & & & & & \\
\hline
\end{tabular}

Whole Sample: $\mathrm{N}=53$, SHIM: $\mathrm{N}=33$, SLIM: $\mathrm{N}=13$

A regression analysis was carried out in order to test whether locus of control is a predictor of after-failure motivation for similar tasks. An $R^{2}=.04, F(1,54)=2.26, p=.138$ emerged. The results are presented in table 4.2.

Table 4.2. Regression analysis of after-failure motivation on locus of control (Criterion: Scores of after-failure motivation).

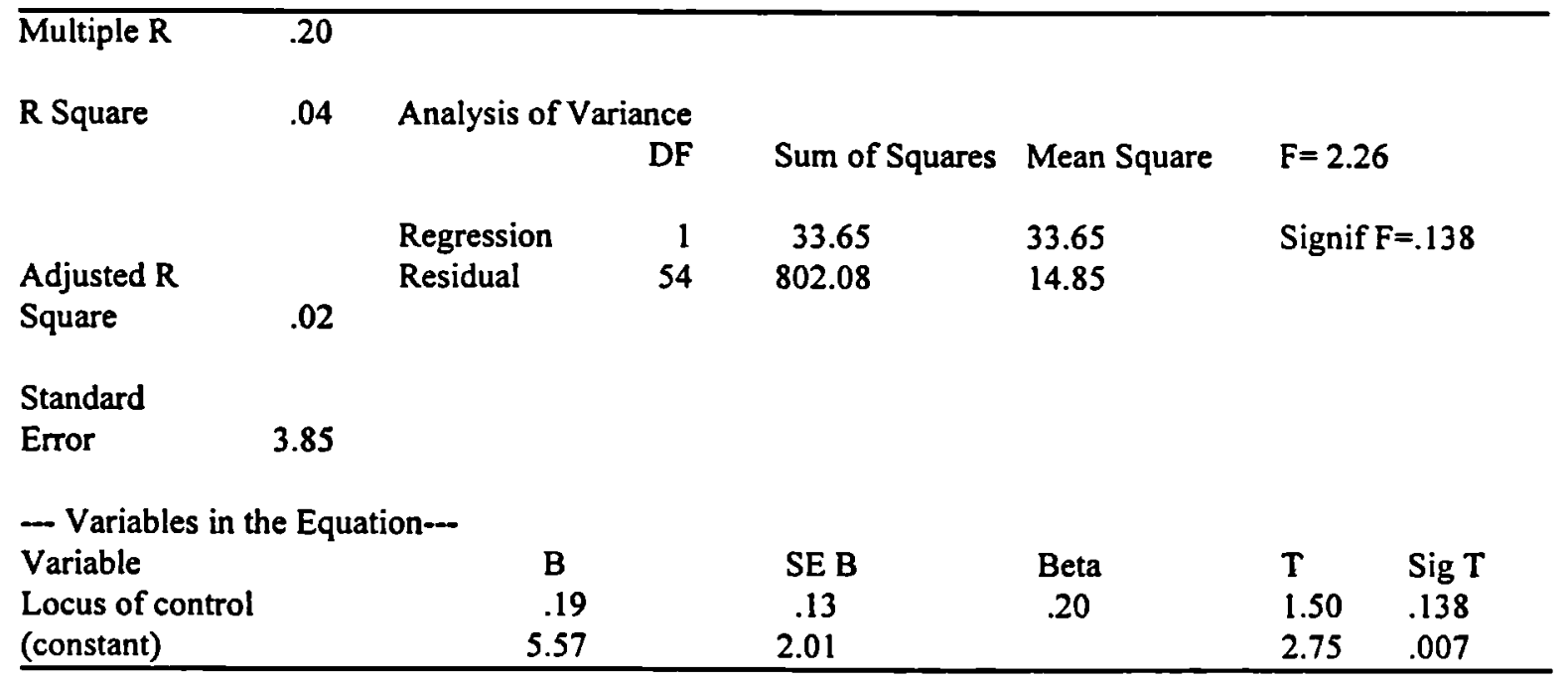

$\mathrm{N}=56$ 
The results showed that locus of control did not predict after-failure motivation for similar tasks. Thus, it can not be argued that internally controlled attributions determine the level of after-failure motivation to engage in a similar task.

In addition, a regression analysis was conducted in order to test the effect of causal stability on after-failure motivation for similar tasks. A square correlation coefficient of $R^{2}=$ $.03, \mathrm{~F}(1,54)=1.95, \mathrm{p}=.168$ was found.

Table 4.3. Regression analysis of after-failure motivation on causal stability (Criterion: Scores on after-failure motivation).

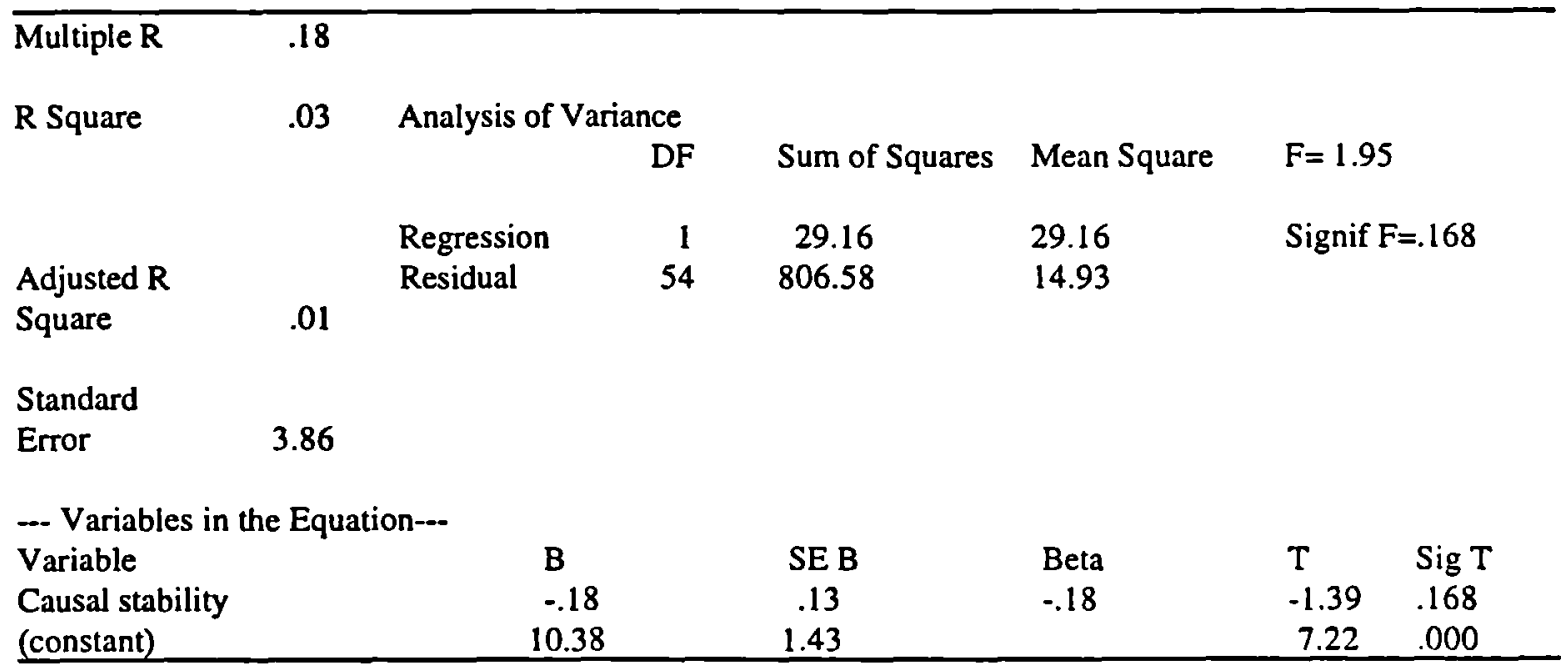

$N=56$

This analysis showed that causal stability did not predict after-failure motivation. That is, whether respondents provided stable or unstable attributions for their failure had no effect on the levels of subsequent motivation.

In order to test the hypothesis that causal stability is a good predictor of after-failure motivation for similar tasks provided that the initial level of motivation is high, while locus of control is not, the sample was divided in two subgroups, high and low initial motivation, and four regression analyses were carried out. Responses above the median value were classified into the high initial motivation group, and those below the median were placed in the low initial 
motivation group. In the first regression analysis after-failure motivation was regressed on causal stability for the subgroup of high initial motivation and a squared correlation coefficient of $R^{2}=.12, F(1,33)=4.54, p=.040$ emerged. The results are presented in table 4.4 .

Table 4.4. Regression analysis of after-failure motivation on causal stability (Criterion: Scores on after-failure motivation) for the subgroup of high initial motivation.

\begin{tabular}{|c|c|c|c|c|c|c|c|}
\hline Multiple R & .34 & & & & & & \\
\hline \multirow[t]{3}{*}{ R Square } & .12 & \multicolumn{6}{|c|}{ Analysis of Variance } \\
\hline & & & DF & Sum of Squares & \multicolumn{2}{|c|}{ Mean Square } & $F=4.54$ \\
\hline & & Regression & 1 & 28.87 & \multicolumn{2}{|l|}{28.87} & Signif $F=.04$ \\
\hline Adjusted $\mathbf{R}$ & & Residual & 33 & 209.50 & \multirow{2}{*}{\multicolumn{2}{|c|}{6.34}} & \\
\hline Square & .09 & & & & & & \\
\hline \multicolumn{8}{|l|}{ Standard } \\
\hline Error & \multicolumn{7}{|l|}{2.51} \\
\hline \multicolumn{8}{|c|}{--- Variables in the Equation--. } \\
\hline Variable & & \multicolumn{2}{|c|}{ B } & SE B & Beta & $T$ & $\operatorname{Sig} \mathrm{T}$ \\
\hline Causal stability & & \multicolumn{2}{|c|}{-.25} & .11 & \multirow[t]{2}{*}{-.34} & -2.13 & .040 \\
\hline (constant) & & & & 1.21 & & 10.61 & .000 \\
\hline
\end{tabular}

$\mathrm{N}=35$

Under the high initial motivation condition causal stability has an effect on after-failure motivation. More specifically, stable attributions are associated with low levels of after-failure motivation, whereas unstable attributions are related to high levels of after-failure motivation to engage in similar tasks.

In the second regression analysis after-failure motivation was once again regressed on causal stability, but this time only the low initially motivated respondents were included in the analysis. A squared correlation coefficient of $R^{2}=.02, F(1,12)=.35, p=.562$ emerged. 
Table 4.5. Regression analysis of after-failure motivation on causal stability (Criterion: Scores of after-failure motivation) for the subgroup of low initial motivation.

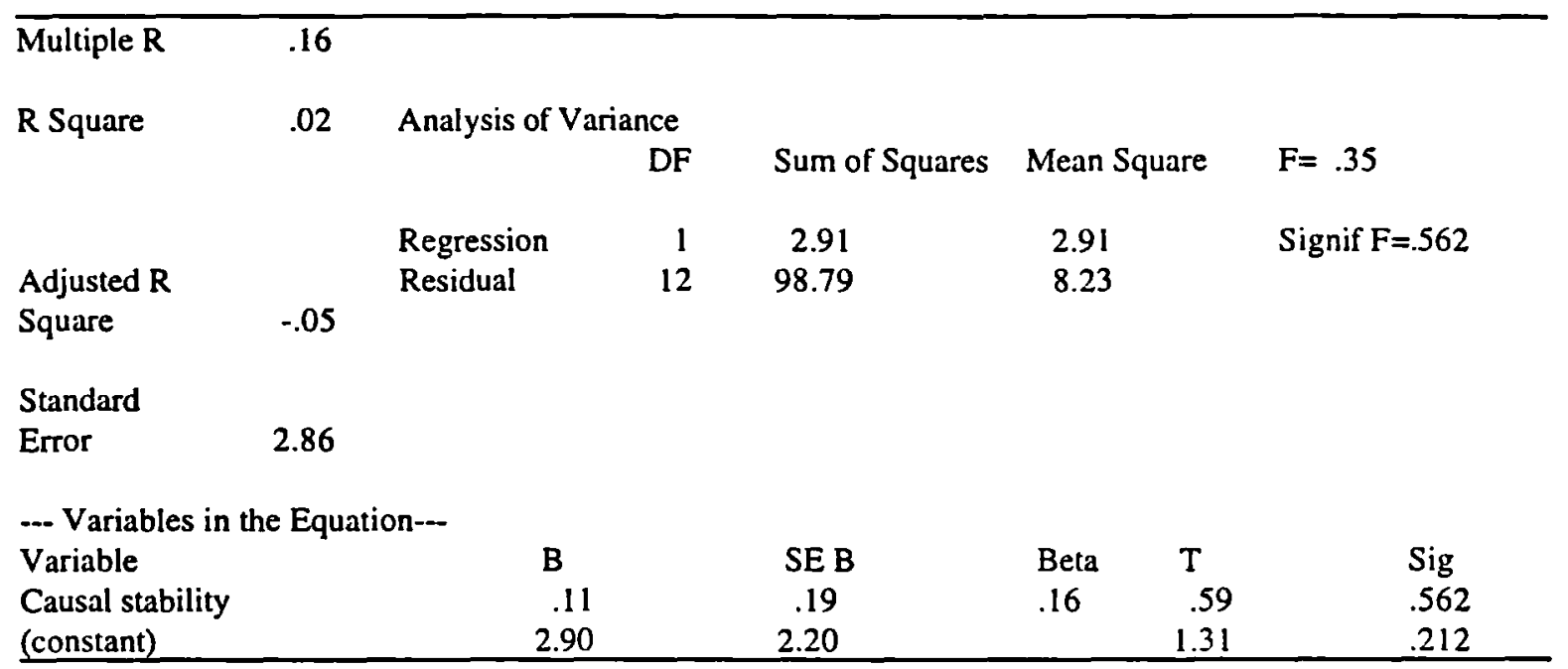

$\mathrm{N}=14$

For the subgroup of low initial motivation, causal stability did not have an effect on after-failure motivation. Thus, when respondents were not interested in solving the radiation problem the stability of the attributions for their failure did not influence subsequent motivation to solve similar problems.

The third and the fourth regression analyses were carried out to test whether locus of control had a different effect on after-failure motivation depending on whether the respondents were in the group of high or low initial motivation (as was demonstrated in the case of causal stability). For the highly motivated group a squared correlation coefficient of $R^{2}=.01, F(1,33)=$ $.55, p=.462$ resulted, while for the low motivation group the squared correlation coefficient was $R^{2}=.00, F(1,12)=.00, p=.990$. Tables 4.6 and 4.7 present the results of regressing afterfailure motivation on locus of control for the conditions of high and low motivation, respectively. 
Table 4.6. Regression analysis of after-failure motivation on locus of control (Critcrion: Scores on after-failure motivation) for the subgroup of high initial motivation.

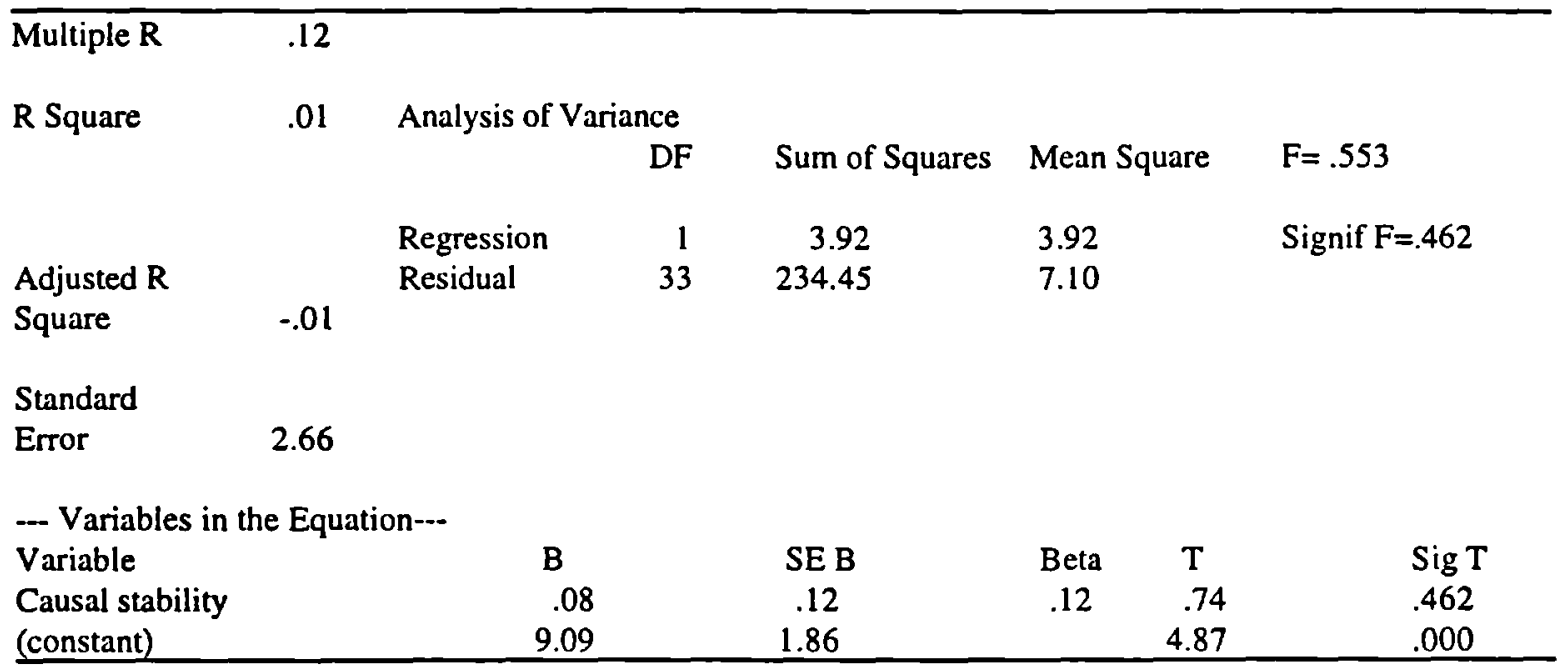

$N=35$

Table 4.7. Regression analysis of after-failure motivation on locus of control (Criterion: Scores on after-failure motivation) for the subgroup of low initial motivation.

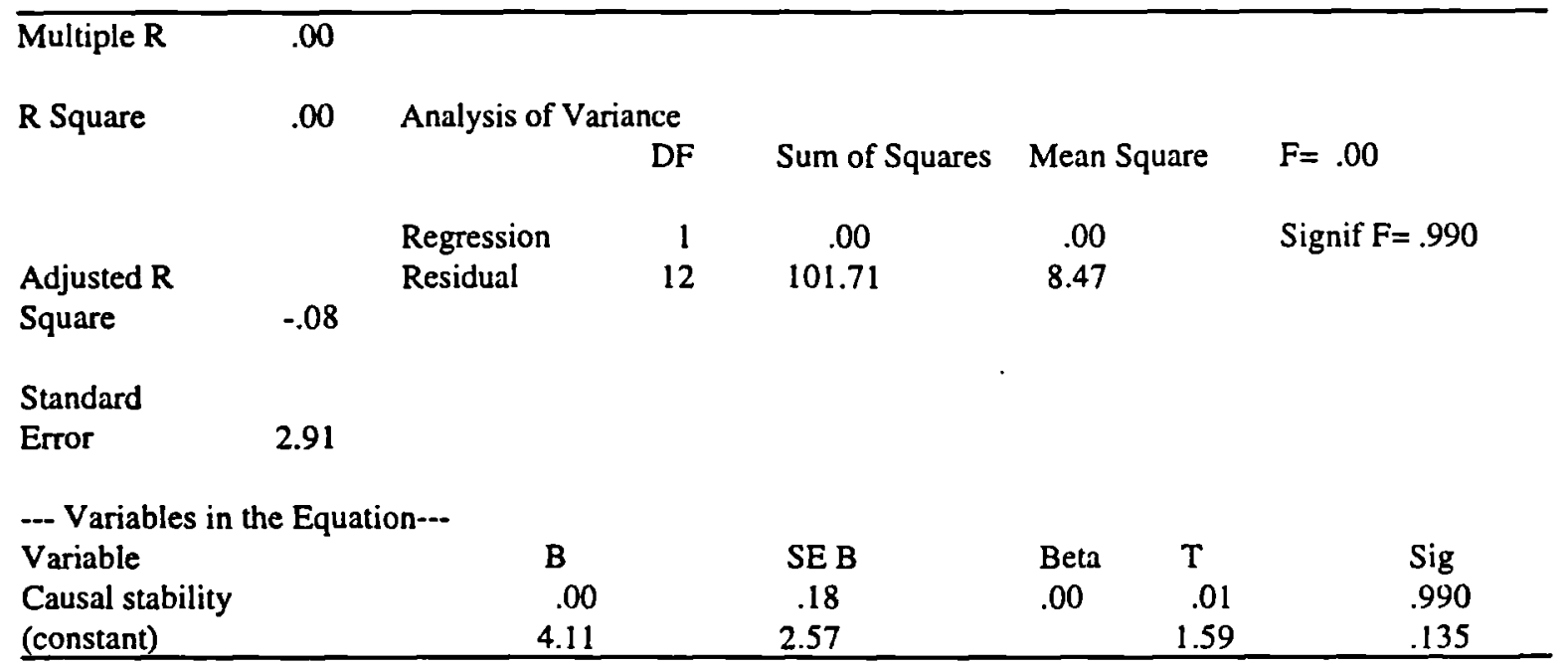

$\mathrm{N}=14$

The results of the two last regressions indicated that locus of control is not significantly predictive of after-failure motivation for similar tasks under any of the conditions of initial motivation. Overall, when failure has been encountered locus of control was shown to have no effect on subsequent motivation to engage in similar tasks. 


\section{DISCUSSION}

Even though comparisons between social learning theory and Weiner's theory have been carried out in the past (see for example, Weiner, Nierenberg, and Goldstein, 1976), their focus was on testing whether locus of control or causal stability was a predictor of expectancies rather than motivation. In an attempt to emphasise the direct effect of causal dimensions on subsequent motivation, this study examined whether locus of control or causal stability predict after-failure motivation to engage in similar tasks.

The results of this study showed that locus of control was not a predictor of afterfailure motivation to engage in similar tasks. Therefore, the suggestion of social learning theory that reinforcement affects future motivation only in the case that reinforcement is perceived as contingent upon the person's own behaviour or attributes is not supported by the findings. The perception of the causes of failure, either as internally or externally controlled, had no influence on after-failure motivation. On the other hand, Weiner's attributional theory was partially confirmed. The stability of the cause of failure was shown to be a predictor of afterfailure motivation provided that the level of initial motivation to engage in the task was high.

The results of this study contradict the predictions from social learning theory and support Weiner's attributional theory of achievement motivation provided that the level of initial motivation is taken into account. Weiner's theory suggests that causal stability of failure is associated with motivation for similar tasks. More specifically, if the cause of failure is expected to re-occur subsequent motivation decreases, whereas if there is no such expectation motivation remains the same or might even increase. However, the results of this study show that when the initial level of motivation is not considered, causal stability does not predict after-failure motivation. Causal stability is a good predictor of subsequent motivation only in 
the case of initially motivated people. In other words, Weiner's theory is not confirmed unless the level of initial motivation is high.

Thus, for Weiner's theory to be valid it is important that failure has been encountered in a task that is perceived by the person to be interesting and challenging in the first place. When the task is initially interesting and challenging, then failure probably leads to a spontaneous search for the causes of this outcome and the stability of these causes has an effect on subsequent motivation for similar tasks. An interesting task that is not accomplished can lead to either stable or unstable attributions for failure. Stable attributions, for example lack of aptitude, are related to lower levels of subsequent motivation, while unstable attributions, for example lack of effort, are related to the maintenance of subsequent motivation. On the other hand, causal stability has no power to predict after-failure motivation when the person thinks that the task is uninteresting. That is, the stability of the causes of failure does not determine the levels of after-failure motivation when people are not stimulated by the task.

To illustrate further the importance of the level of initial motivation for the confirmation of Weiner's attributional theory an example of failing will be utilised. Thus, lets assume that two people fail in a specific task. Both of them attribute their failure to lack of effort, which is considered by attributional research to be an unstable cause. According to the predictions of Weiner's attributional theory, these people's motivation for similar tasks will remain at the same levels merely because lack of effort is something unstable. However, let's also assume that one of these people is highly motivated to carry out the task, while the other one thinks the task not to be interesting and challenging. For the highly motivated person attribution of failure to lack of effort leads to maintenance or even increase of motivation because the cause of failure is changeable and the person is interested in changing it. For the person who finds the task to be dull, attribution to lack of effort is perceived as a changeable 
cause, but he/she is not interested in changing this cause and thus, levels of motivation can drop as a result of the negative effect of failure. Therefore, it can be concluded that failure is not a negative reinforcement to engage in similar tasks when its causes are perceived as changeable and the person is willing to change them.

According to Feather's (1982) expectancy-value model, achievement motivation is related to the expectancy that specific actions will lead to certain outcomes and to the valence of these outcomes. Feather has focused on the effect of valences of objects, events, and activities on motivation to engage in specific tasks. The finding of this study that the initial level of motivation moderates the relationship between causal stability and after-failure motivation can be explained by Feather's expectancy-value theory. The level of people's initial motivation to engage in a task is related to the valences of the specific outcomes of the task. Therefore, the higher the valences of possible outcomes the higher the initial motivation to engage in the task.

On the basis of these findings, it is argued that Weiner's attributional model has to incorporate the initial level of motivation to engage in a task as a moderator of the relation between causal stability of failure and subsequent motivation for similar tasks. As far as failure is concerned, Weiner's prediction that causal stability determines motivation is valid provided that the task at hand is interesting and challenging.

In this study the effect of attributions for failure on subsequent motivation for similar tasks was examined and more specifically, whether locus of control and/or causal stability are predictors of after-failure motivation. It was demonstrated that locus of control failed to predict the level of motivation which follows an event of failure, while causal stability was shown to influence subsequent motivation in the case of highly motivated respondents. Therefore, the findings support a proposition for the refinement of Weiner's theory for the case 
of failure, as well as the superiority of this refinement of Weiner's theory to the predictions of social learning theory. 
CHAPTER FIVE

A PROPOSITION FOR A MORE

RELIABLE AND VALID MEASURE

OF ATTRIBUTIONAL STYLE 


\section{INTRODUCTION}

In order to examine one of the main hypothesis of this thesis, which is whether attributional style has an effect on the formulation of situational attributions, an important preliminary task in the present research concerns addressing the issues of the conceptualisation, as well as the actual measurement, of attributional style.

The concept of attributional (or cognitive) style was discussed by Abramson, Seligman, and Teasdale (1978) in the reformulated model of learned helplessness and was defined as the habitual way people explain positive or negative events in their lives. In particular, the causes that each person selects to attribute his/her successes or failures are supposedly most of the time the same, as far as the causal dimensions of internality, stability, and globality are concerned. According to Abramson, Seligman, and Teasdale (1978), "when people believe that outcomes are more likely or less likely to happen to themselves than to relevant others, they attribute these outcomes to internal factors" (p. 52). Stability refers to the fact that a cause is seen as enduring and always activated on the one hand, or transient and not permanent on the other. Finally, a cause is global when it is perceived to occur in a broad range of situations.

Seligman (1990) refers to attributional style as a developmentally acquired personality characteristic and proposes two types of style: the optimistic explanatory style (OES) and the pessimistic explanatory style (PES). People who are characterised by an OES usually attribute failures to external, unstable, and specific causes and successes to internal, stable, and global ones. On the other hand, people who have a PES often attribute bad events to internal, stable, and global factors and good events to external, unstable, and specific causes. Seligman (1990) also suggests that attributional style "stems from your view of your place in the world-whether you think you are valuable and deserving, or worthless and hopeless" (p. 44), directly relating 
attributional style to self-esteem.

There are, however, a number of studies that, although they did not directly address the issue of whether a person who attributes negative events to internal, stable, and global factors, will make external, unstable, and specific attributions for positive events, they provided a number of findings that are not consistent with this theoretical assumption of the reformulated model. Hull and Mendolia (1991) used structural modelling techniques to test the mediational role of expectancies in the relation between attributions and depressive affect. Since the specification of attributional style was particularly problematic within Lisrel VI, they modelled it separately in an attempt to appropriately specify its structure. The best fitting model hypothesised that attributions for positive and negative events do not form a single latent variable; that is, internal, stable, and global attributions for positive outcomes on the one hand, and internal, stable, and global attributions for negative outcomes on the other, do not seem to be polar opposites of the same underlying dimension. Moreover, Hull and Mendolia's (1991) finding that attributions for positive outcomes were only indirectly related to depression by means of expectancies, whereas attributions for negative outcomes were both directly and indirectly related to depression can be interpreted as an indication that attributional style for positive and negative outcomes are separate variables.

Ahrens and Haaga (1993) in a study of attributional style, expectancies, affectivity, anxiety, and depression provide an intercorrelation between the scores on the six positive scenarios drawn from the Attributional Style Questionnaire (ASQ; Peterson et al., 1982) and the scores on the Expanded Attributional Style Questionnaire (EASQ; Peterson \& Villanova, 1988) which contains only negative events. The positive attributional style measured by the six positive scenarios of ASQ (Peterson et al., 1982) was not significantly correlated with negative attributional style measured by the EASQ (Peterson \& Villanova, 1988). Moreover, Corr and 
Gray (1994) in a one-year predictive validity study investigating the role of attributional style, socialisation, and cognitive ability factors in insurance sales performance reported a nonsignificant correlation between the combined positive events scale and the combined negative events one of the ASQ. These findings suggest that attributional styles for positive and negative events might well be independent of each other; specifically that the people who attribute positive events to internal, stable, and global causes do not necessarily attribute negative events to external, unstable, and specific causes - in contrast to the reformulated model (Abramson, Seligman, \& Teasdale, 1978) and Seligman's (1990) definition of attributional style.

It is possible that certain people who attribute unfavourable outcomes to external, unstable, and specific factors do not tend to attribute positive outcomes to internal, stable, and global causes. This might be the case because a high value placed on realism or modesty has an effect on the process of making attributions. Taking complete responsibility for negative outcomes and viewing them as stable and global can be perreived as a pathetic reaction to misfortune and, therefore, prevents people from attributing negative events to internal, stable, and global factors. Attributing good events to one's self and considering them as stable and global might be moderated by modesty and a sense of realism. The literature on the partial independence of positive and negative affect suggests that optimism is not necessarily the opposite of pessimism. For example, Argyle and Henderson (1985), in the field of marital satisfaction, showed that positive and negative affect can be independent of each other. Partners can have strong positive feelings (i.e., related to frequency of intercourse), as well as negative ones (i.e., related to the frequency of rows). In the same way, the simultaneous manifestation of optimism and pessimism by an individual is possible within a specific area of his/her life, for example, at work. Hence, it is important to find a way around the problem of 
using events that are representative of a specific domain in order to be able to measure the tendency of a person to be generally optimistic or pessimistic, as far as this specific domain is concerned.

Another issue concerning attributional style involves internality as a component of attributional style. Even though the reformulation of the learned helplessness model (Abramson, Seligman, \& Teasdale, 1978) distinguishes between the concepts of personal and universal helplessness and suggests that a person can be internally or externally helpless, rather confusingly internality is still incorporated as a causal dimension of a vulnerable attributional style. The reformulated model suggests that "attributing the cause of helplessness internally often, but not always, implies a grimmer future than attributing the cause externally, since external circumstances are usually but not always in greater flux than internal factors" (p.56). However, it has been suggested that one can feel helpless in face of the future without feeling that the future will necessarily be bad (Weiner, 1986; Brewin, 1988). Weiner (1986) argues that even though the notion of helplessness is associated with the concept of locus of control, helplessness is not the core theme of pessimism. More specifically, he suggests that a person can feel helpless when he/she perceives lack of personal control in the situation but it is not necessary to feel hopeless as well. The person might believe that an external force will change a negative situation and consequently might be in a state of guarded optimism, certainly not pessimism. Weiner's attributional theory of motivation and emotion proposes that the dimension of causal stability (and perhaps globality) is the one that effects one's expectations for future success or failure, mediating the effect of good or bad outcomes on motivation and emotion, and not the causal dimensions of locus or controllability.

Abramson, Metalsky, and Alloy (1989) have recently introduced the hopelessness theory of depression which is a revision of the reformulated theory of helplessness and 
depression. In their revision they suggest there are individual differences in attributional style, which is defined as a distal factor leading to hopelessness and depression. Some individuals may exhibit a general tendency to use stable and global causes in explaining negative events, while other individuals may not. Those who tend to explain negative events in stable, global terms should have bleak expectations of the future, which, in turn, leads to bad feelings. This tendency is defined as a depressogenic attributional style which provides "specific vulnerability" to depression; but it does not necessarily cause the occurrence of the symptoms. Thus, although many cases of hopelessness depression will occur among cognitively vulnerable people when they are confronted with negative events, people who do not have a depressogenic attributional style also may develop hopelessness depression when they are confronted with events sufficient to engender hopelessness. Hopelessness theory focuses on the role of stability and globality in the formulation of expectations, while de-emphasising the causal dimension of internality.

It should be made clear that the hopelessness theory predicts not only depression, but also how people can maintain a positive emotional state. According to this theory, the occurrence of a negative event provides a challenge to a positive emotional state. The attribution of a negative event to stable and global causes, the perceived importance of the event's consequences, the inference of negative characteristics about the self, and finally, a negative attributional style all contribute to the occurrence of hopelessness and, as a result, to the disturbance of a positive emotional state. In contrast, refraining from making these inferences, as well as the existence of a positive attributional style, should ensure positive affect. Consequently, attributional style is not a sufficient cause leading to depression, as the theory also specifies other factors which determine whether a negative event causes hopelessness and depression. 
Although Cutrona, Russell, and Jones (1985) questioned whether most individuals are sufficiently consistent across situations in the causal attributions that they make to justify the concept of attributional "style", they provided evidence of a stronger cross-situational consistency of the stability and the globality dimensions compared to the dimension of internality. Cutrona et al. (1985) used a confirmatory factor analysis to test the construct validity of the ASQ (Peterson et al., 1982). The best fitting model hypothesised both crosssituational consistency and situational specificity to underlie their dataset. The results suggested little evidence of cross-situational consistency for the internality dimension. This finding is consistent with the low levels of internal reliability reported for this subscale (.33). The results for stability and globality provided stronger evidence of cross-situational consistency.

At this point one must consider the fact that the locus of control scales (Furnham \& Steele, 1993) are more reliable compared to the internality dimension subscale of the attributional style questionnaires. It can be argued that the locus of control scales generally have more items which contribute to their greater reliability. However, even in the case of the EASQ, which is an attempt to increase the internal consistency of the attributional style questionnaires by adding more items, the alphas of negative stability and globality had higher coefficients ( .85 and .88 , respectively) compared to the negative internality dimension (.66). Thus, the paradox might not only be explained by the number of items, but also by the different nature of the items contained in these scales. More specifically, in the locus of control scales the respondents are asked to rate a number of locus of control beliefs, for example to what extent they agree that "Making money is a matter of good fortune" (item taken from Spector's WLC scale), while the attributional style questionnaires ask respondents to vividly imagine an event that might or might not have happened to them. In the case of the attributional style 
questionnaires the event items cause the retrieval of a more complex causality representation where single factors can not be perceived as sufficient for explaining an outcome, and because subjects are asked to rate only one cause they tend not to be consistent across situations. It seems that attributions to internal or external factors are not necessarily perceived by the subjects as mutually exclusive. The relevant literature has shown that subjects are more likely to employ combinations of both internal and external attributions for complex interpersonal events (Bradbury \& Fincham, 1988). On the other hand, locus of control beliefs do not lead to complex causal schemata as the person is not asked to find the causes of an event but rather to state the extent to which the statement is in accordance with his/her belief system.

Finally, the superiority of attributional style for negative events over attributional style for positive events as a more reliable and valid measure of performance can be argued on the basis of findings in the relevant literature.

Burns and Seligman (1989) analysed attributional style across life spans. Subjects, whose average age was 72, completed the ASQ (Peterson et al., 1982) and provided diaries and letters written in their youth, an average of 52 years earlier. The results showed that attributional style for negative events was stable throughout adult life, but their attributional style for positive events was not stable across this interval. Furthermore, Burns and Seligman (1989) reported that the stability and the globality dimensions showed intra-subject consistency, while the internality dimension was less consistent.

Seligman and Schulman (1986), looking at the influence of attributional styles on the performance of life insurance sales agents, found that low scores on negative attributional style (external, unstable, and specific attributions for negative outcomes) were correlated with higher productivity, whereas positive attributional style was not. Moreover, Peterson (1991) suggested that attributional style for good events might not yield rich data because people are 
less "mindful" in thinking about good events and, therefore, offer unrevealing, stereotyped responses to them. This speculation is suggestive of Weiner's attributional paradigm (1986) in which he proposes that causal research is more likely undertaken when an event is negative, unexpected, or important.

The aim of this study is to propose a more reliable and valid measure of attributional style by focusing on the dimensions of stability and globality for negative events. In order to do that the following hypotheses were tested: (1) there are not significant negative correlations between attributions for positive and attributions for negative outcomes, (2) attributions for positive and attributions for negative outcomes do not load on three correlated factors, namely, internality, stability, and globality, in an oblique factor analytic investigation of their nature, (3) the causal dimension of internality does not show acceptable levels of cross-situational consistency, while stability and globality show acceptable levels of cross-situational consistency, and (4) the causal dimensions of stability and globality for negative events are valid measures of the concept of pessimism and confidence.

\section{METHOD}

Respondents. A total of 189 insurance sales staff from a British financial organisation took part in this study. There were $34(18 \%)$ women and $155(82 \%)$ men in the group. They ranged in age from 22 to 53 years (the mean being 31 years, $S D=5$ years). 3.7 percent had a basic salary of $£ 14,000$ to $£ 15,999$ per year, 70.4 percent $£ 16,000$ to $£ 17,999,21.8$ percent $£ 18,000$ to $£ 19,999$, and 3.7 percent had a salary of more than $£ 20,000$ per year. The respondents received a profile of their own results with an interpretive guide after the study. Selling insurance is a job particularly suitable for the investigation of attributional style because sales 
staff repeatedly encounter failure, rejection, and indifference from prospective clients. Consequently, the turnover rate among insurance agents is very high (Seligman \& Schulman, 1986).

Questionnaires. Participants completed the Occupational Attributional Style Questionnaire (OASQ; Furnham, Sadka, \& Brewin, 1992) and the Social Problem Solving Inventory (SPSI; D'Zurilla \& Nezu, 1990). For the measurement of attributional style the OASQ (Furnham, Sadka, \& Brewin, 1992) was preferred to the ASQ (Peterson et al., 1982) because it is specifically related to the work setting. The Negative Problem Orientation subscale of the SPSI, which is a measure of pessimistic orientation in problem solving, was used for the concurrent validation of OASQ mainly for three reasons: firstly, because the subscale measures a motivational component of problem solving and learned helplessness theory suggests that attributional style affects motivation; secondly, because motivation is assumed to be necessary for performance; and thirdly, because problem solving is part of the job of selling insurance which requires the sales staff to find the best fit between client financial needs and the organisation's financial services. Respondents' scores on the Rapid Personality Questionnaire (RPQ; Rust, 1991) were also available. This instrument is used by the organisation in the selection of insurance agents.

Occupational Attributional Style Questionnaire (Furnham, Sadka, \& Brewin, 1992). The OASQ which was closely modelled on the ASQ presents subjects with 8 different situations concerning work life, half of which are positive events (e.g., getting a promotion), while the other half are negative (e.g., your boss acts aggressively towards you). Subjects are required to imagine vividly themselves in the situation described, write down the most likely cause of 
the situation, and finally, rate this cause on separate seven-point scales for nine causal dimensions, namely, internality, stability, globality, externality, chance, personal control, colleague control, foreseeability, and importance. One of the advantages of this questionnaire compared to other methods of attribution research concerns the fact that subjects are asked to find their own causes of events, not simply to choose one from a provided list of causes. According to Wimer and Kelley (1982), the process of thinking about and arriving at an attribution may provide a fuller or possibly clearer understanding of the meaning of a cause than in the more passive case when one simply reads an attribution.

The OASQ was found to have alphas ranging from .52 to .84 and test-retest reliability for a four-week period yielded $r=.87$. The coefficients of internal consistency obtained for the current study's data are presented in the results section. As far as construct validity is concerned, attributions for positive events particularly (and to a lesser extent with positive and negative combined) were significantly and consistently correlated with salary, job satisfaction, intrinsic motivation, and social class-variables that previous research has demonstrated to be correlated with optimistic attributional style. In this study the dimensions of internality, stability, and globality were used.

Social Problem Solving Inventory (D'Zurilla \& Nezu, 1990). The SPSI was developed by D'Zurilla and Nezu (1990) and is based on the theoretical framework of a problem solving model. According to this model, social problem solving is a complex cognitive-affectivebehavioral process that consists of two major components: (a) problem orientation and (b) problem solving skills, which compose the two main scales of the questionnaire.

Problem Orientation refers to the motivational component of the problem solving process, arising primarily from past problem solving experiences. There are two types of 
problem orientation, positive problem orientation (PPO) and negative problem orientation (NPO). For the purposes of this study the subscale of NPO was used. The subscale of NPO (10 items) concerns the extent to which a person is vulnerable to anxiety, self-doubt, has low self-efficacy, and a tendency to see things in a pessimistic way at early signs of poor outcomes. Test-retest reliability (average of three weeks apart) for the whole questionnaire and the Problem Orientation Scale were .87 and .83 , respectively (D'Zurilla \& Sheedy, 1991). Alpha coefficients for the same measures are .94 and .94 , respectively (D'Zurilla \& Sheedy, 1991). The coefficient of internal reliability for the NPO subscale obtained in the current study was .88 .

Rapid Personality Questionnaire (Rust, 1991). The RPQ contains eighty items which are designed to elicit a spectrum of the individual's personality profile in occupational settings. The items are adjectives rated on separate five-point scales. The subjects are required to respond to the items in terms of how they think each item applies to them while at work. The questionnaire contains five scales, namely, confidence, extraversion, tough-mindedness, conformity, and being structural. Regarding the reliability of the instrument there are reported coefficients of split-half reliability ranging from .76 to .84 , coefficients of internal consistency ranging from .76 to .86 , and test-retest reliability coefficients ranging from .85 to .93 (Rust, 1991). According to Rust (1991), the factorial validity of the RPQ is demonstrated by the fact that its five factor solution matches the five domains of the test specification, as well as it conforms to the "big five" solution by McCrae and Costa (1987). In the current study the total score on the 16-item scale of confidence was used. The coefficient of internal consistency for the present data could not be computed because the researcher had only the total score of each subject on the scale and not the scores on each of the sixteen items of the scale. 
Procedure. The respondents completed the questionnaires at work. As a motivational incentive towards accurate responding the respondents were promised and received an individualised bar graph profile and a guide to enable them to interpret their own scores. A total of 189 questionnaires were returned; this is a response rate of 54 per cent which is considered to be an average response rate.

\section{RESULTS}

Descriptive statistics, coefficients of alpha reliability, and the intercorrelations between the OASQ's six scales for both positive and negative events are presented in table 5.1. There are moderate positive correlations among the scales of positive internality, positive stability, and positive globality at a .001 level of significance. Moreover, the scales measuring the causal dimensions for negative events show moderate positive intercorrelations at a .05 and .001 level of significance. These positive correlations are in accordance with Seligman's conceptualisation of attributional style in which the dimensions of internality, stability, and globality are assumed to be related to one other.

Table 5.1. Descriptive statistics, Alphas, and Zero-Order/Partial correlation coefficients between the OASQ's scales.

\begin{tabular}{|c|c|c|c|c|c|c|c|c|c|}
\hline \multirow{2}{*}{$\begin{array}{l}\text { Dimensions } \\
\text { Positive }\end{array}$} & \multirow[t]{2}{*}{$\mathbf{M}$} & \multirow[t]{2}{*}{ SD } & \multirow[t]{2}{*}{ Aipha } & \multicolumn{2}{|c|}{ +Internality } & \multirow[t]{2}{*}{ +Stabılity } & \multirow[t]{2}{*}{ +Globality } & \multirow[t]{2}{*}{-Internality } & \multirow[t]{2}{*}{-Stability } \\
\hline & & & & & & & & & \\
\hline $\begin{array}{l}\text { 1. Intemality } \\
\text { 2. Stability } \\
\text { 3. Globality }\end{array}$ & $\begin{array}{l}15.8 \\
14.6 \\
13.3\end{array}$ & $\begin{array}{l}1.5 \\
3.0 \\
3.6\end{array}$ & $\begin{array}{l}.28 \\
.72 \\
.65\end{array}$ & $\begin{array}{l}.40^{* *} \\
.33^{* *}\end{array}$ & $\begin{array}{l}\left(.41^{* * *}\right) \\
\left(.33^{* * * *}\right)\end{array}$ & $.56^{* * *}\left(.53^{* * *}\right)$ & & & \\
\hline \multicolumn{10}{|l|}{ Negatıve } \\
\hline $\begin{array}{l}\text { 4. Intemality } \\
\text { 5. Stability } \\
\text { 6. Globality } \\
\end{array}$ & $\begin{array}{l}15.3 \\
15.4 \\
10.9\end{array}$ & $\begin{array}{l}3.9 \\
4.1 \\
5.4\end{array}$ & $\begin{array}{l}.40 \\
.78 \\
.67\end{array}$ & $\begin{array}{l}.06 \\
.10 \\
.13^{*}\end{array}$ & $\begin{array}{l}(.05) \\
(.09) \\
\left(.14^{*}\right)\end{array}$ & $\begin{array}{l}.02(.03) \\
.30^{* * *}\left(.31^{* * *}\right) \\
.16^{* *}\left(.17^{* *}\right)\end{array}$ & $\begin{array}{ll}. .07 & (-.05) \\
.03 & (.04) \\
.21^{* * *}\left(.22^{* *}\right)\end{array}$ & $\begin{array}{l}.16^{*} \quad\left(.15^{*}\right) \\
44^{* * *}\left(45^{* * *}\right)\end{array}$ & $42^{* * *}\left(42^{* * *}\right)$ \\
\hline
\end{tabular}

1. *** $p=.001 * * p=.01 * p=.05$

2. (+) stands for positive. (-) stands for negative

3. The scales for positive events consist of 3 items each. while the scales for negarive events consist of 4 items each.

4. Partial correlations controlling for age, sex, and salary in parentheses. 
There are primarily positive intercorrelations among the scales for the positive events and the scales for the negative ones (apart from a low negative correlation -.07 between positive globality and negative internality, which is not statistically significant). Four of these correlations are very low ranging from .02 to .10 and have failed to reach significance. The significant positive correlations are quite low ranging from .13 to .30 . There is not much difference between the partial correlations of OASQ scales controlling for age, sex, and salary and the Zero-Order correlations. Finally, the combined positive events scale, which contains the dimensions of positive internality, stability, and globality, has a low positive correlation .18 $(p<.006)$ with the combined negative events scale (negative internality, stability, and globality). The partial correlation between the combined positive events scale and the combined negative events scale with the variables of age, sex, and salary controlled, is positive and not much higher than the Zero-Order correlation $(.20, \mathrm{p}<.003)$.

It is interesting that firstly, the correlations between the scales for positive and negative events were not negative and secondly, the correlation between the combined positive events and the combined negative events scales was low and positive. This is contrary to the supposition that explanatory style is the habitual way of either attributing successes to internal, stable, global causes and failures to external, unstable, and specific ones or attributing successes to external, unstable, and specific factors and failures to internal, stable, global ones.

In order to test hypothesis 2 a higher-order factor analysis of the OASQ's scales was carried out to examine whether the causal dimensions of internality, stability, and globality for positive and negative events would load on three correlated factors. According to the reformulated model of learned helplessness, internality, stability, and globality are three distinct but related dimensions of causality and should therefore form three separate factors. Moreover, 
attributions for good and bad events of each dimension should load on the same factors, with dimensions for bad events having a negative loading, because according to the reformulated model individuals who attribute positive events to internal, stable, and global factors would attribute negative events to external, unstable, and specific causes. Consequently, the factor analysis should generate three factors, namely, internality, stability, and globality each of which will include the scales of positive and negative internality, stability, and globality, respectively.

A principal components analysis with OBLIQUE rotation and predefined number of factors ( 3 factors) was carried out to see whether attributions for positive and negative events for each dimension would load on the same factors. The three extracted factors accounted for $74.7 \%$ of the common factor variance. The OBLIQUE rotation indicated that the factors were not highly correlated ranging from .08 to -.17 . The results, as well as the correlations between the extracted factors, are presented in table 5.2.

The results of the factor analysis showed that the causal dimensions for positive events loaded on one factor, while the same dimensions for negative events loaded on two other factors. Consequently, the dimensions for positive and negative events form distinct constructs, which are virtually independent from each other (the correlation between factor 1 and factor 2 is $r=.08$ and between factor 1 and factor 3 is $r=-.16$ ). These results provide evidence that a specific attributional style for positive events is not associated with a specific attributional style for negative events. That is, the individuals who attributed positive outcomes to internal, stable, and global causes did not have a tendency to provide external, unstable, and specific causes for negative outcomes. 
Table 5.2. Factor Analysis of the OASQ's scales of internality, stability, and globality for positive and negative events (loadings above 1.401 are shown in boldface).

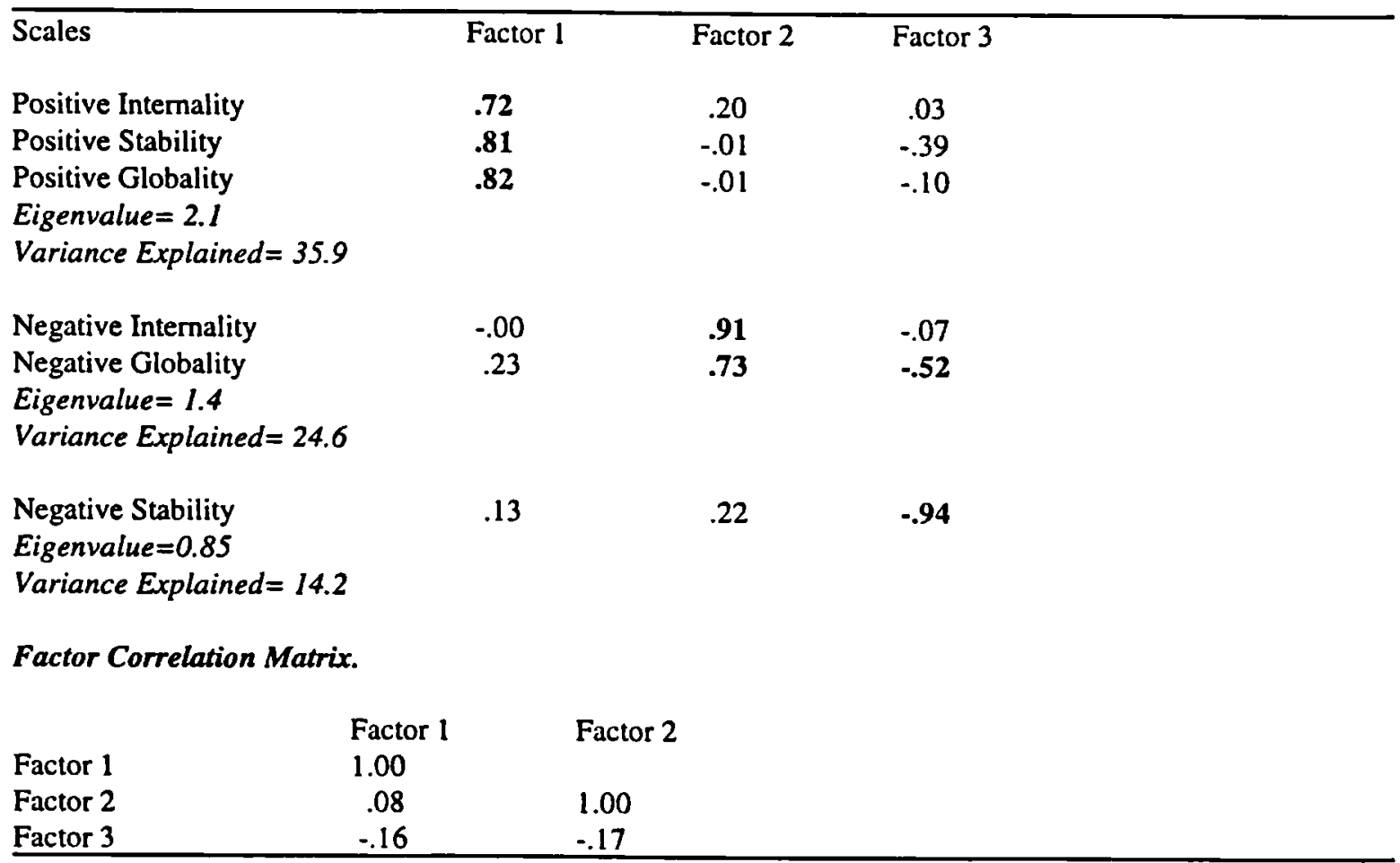

$N=189$

As far as the testing of hypothesis 3 is concerned, the alpha coefficients of internal consistency were calculated for each dimension to see whether the items that are contained in every dimension are highly intercorrelated. The Cronbach alpha coefficients for all the dimensions are presented in table 5.1. The results show that the OASQ has moderately high coefficients of internal consistency for stability and globality, ranging from .65 for positive globality to .72 for positive stability. However, the scale of internality shows quite low coefficients of internal reliability ( .28 for positive internality and .40 for negative internality), which means that the subjects attribution of positive or negative events to internal factors depends on the specific event and is not cross-situational. Therefore, the causal dimension of internality does not appear to demonstrate acceptable levels of within subject consistency, while stability and globality do show stronger cross-situational consistency. After a literature 
review on the concept of attributional style a table was produced that presents a number of other studies' coefficients of alpha reliability showing the same pattern of findings with the current study (table 5.3).

As the attributional style for negative events was shown to be a more reliable and valid measure compared to the attributional style for positive events (see pp. 103-104 of the introduction) two stepwise multiple regressions were carried out in order to test the validity of negative stability and negative globality as measures of pessimism and confidence. Negative stability and globality are predicted to be related to a pessimistic attitude towards the confrontation of problems and low levels of confidence. Table 5.4 presents the correlations among OASQ's negative stability, OASQ's negative globality, SPSI's negative problem orientation (NPO), and RPQ's subscale of confidence.

Table 5.4. Pearson correlations among negative stability (NS), negative globality (NG), NPO, and RPQ's subscale of confidence (CON).

\begin{tabular}{llll}
\hline & NS & NG & NPO \\
NS & & & \\
NG & $.42^{* * *}$ & & \\
NPO & .07 & $.19^{* *}$ & $-.65^{* * *}$ \\
CON &. .13 & $-.22^{* *}$ & \\
\hline
\end{tabular}

$* * * p=.001 \quad * * p=.01$

In the first regression analysis, the SPSI's subscale NPO was used as the dependent variable, while the independent variables were the negative stability and the negative globality scales. A stepwise multiple correlation coefficient of $R=.19, F(1,187)=7.50, p<.006$ was found between the NPO subscale and the predictor, namely, negative globality (table 5.5). The stepwise method was used because negative stability and globality are correlated $(r=.42)$ and, therefore, it is likely that there is a portion of the dependent variable's variance that is explained 


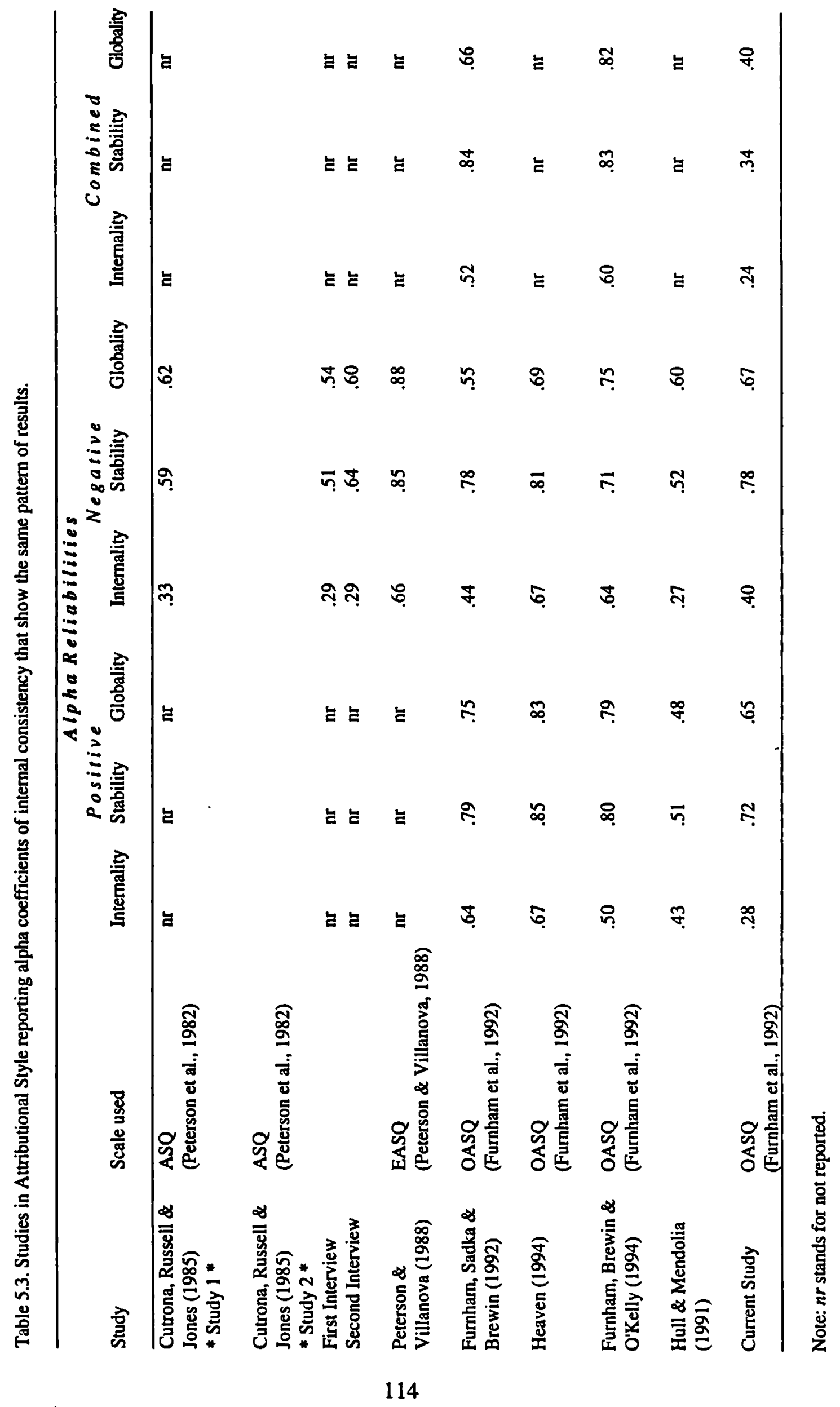


by both the predictors. By using the stepwise method the commonly explained variance is given to the predictor which has the bigger unique explained variance. Another regression method that can be used is the forced entry method which allow us to report the effect of each predictor on the criterion after controlling for the effect of the other predictor. In the forced entry method the commonly explained variance is not accounted by either the predictors.

Table 5.5. Multiple regression of the NPO Scale (Criterion: Scores on the NPO Scale).

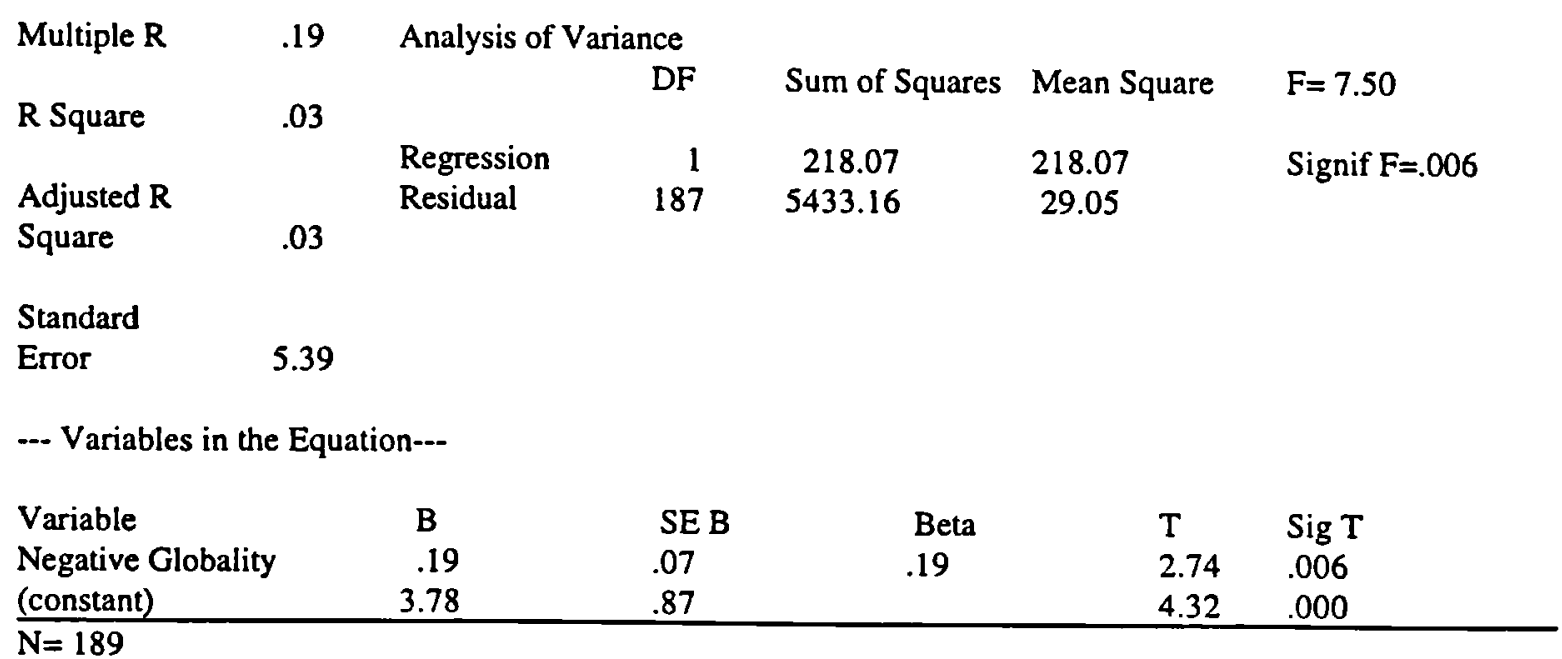

Finally, a stepwise multiple regression was conducted using the RPQ's scale of confidence as the criterion and the scales of negative stability and negative globality as the predictor variables. Confidence is used interchangeably in the literature with the concept of self-esteem, which according to the Seligman (1990) determines attributional style. Actually, attributional style is a result of how valuable and deserving one perceives himself/herself to be. Table 5.6 presents the stepwise multiple regression results. A multiple correlation coefficient of $\mathrm{R}=.21, \mathrm{~F}(1,187)=9.23, \mathrm{p}<.002$ was generated between the dependent variable, namely, the RPQ's scale of confidence and the predictor which succeeded in entering the regression equation, namely, negative globality. 
Table 5.6. Multiple regression of the RPQ's Scale of Confidence (Criterion: Scores on the Confidence Scale).

\begin{tabular}{|c|c|c|c|c|c|c|}
\hline \multirow[t]{2}{*}{ Multiple R } & \multirow[t]{2}{*}{.21} & \multicolumn{2}{|c|}{ Analysis of Variance } & \multirow{3}{*}{ Sum of Squares } & \multirow{3}{*}{ Mean Square } & \multirow{3}{*}{$F=9.23$} \\
\hline & & & DF & & & \\
\hline R Square & .04 & & & & & \\
\hline $\begin{array}{l}\text { Adjusted R } \\
\text { Square }\end{array}$ & .04 & $\begin{array}{l}\text { Regression } \\
\text { Residual }\end{array}$ & $\begin{array}{r}1 \\
187\end{array}$ & $\begin{array}{c}119.05 \\
2410.39\end{array}$ & $\begin{array}{r}119.05 \\
12.88\end{array}$ & Signif $F=.002$ \\
\hline $\begin{array}{l}\text { Standard } \\
\text { Error }\end{array}$ & 3.59 & & & & & \\
\hline $\begin{array}{l}\text {..- Variables in the } \\
\text { Variable } \\
\text { Negative Globality } \\
\text { (constant) }\end{array}$ & e Equ & $\begin{array}{l}\text { B } \\
-.- \\
-.14 \\
12.80\end{array}$ & $\begin{array}{l}\text { SE B } \\
.04 \\
.58\end{array}$ & $\begin{array}{l}\text { Beta } \\
-.21\end{array}$ & $\begin{array}{c}\mathrm{T} \\
-3.0 \\
21.93\end{array}$ & $\begin{array}{l}\text { Sig T } \\
.002 \\
.000\end{array}$ \\
\hline
\end{tabular}

$\mathrm{N}=189$

The data shows the best predictor of negative problem orientation to be the causal dimension of negative globality suggesting that individuals with high scores on negative globality tend to have a pessimistic attitude towards the confrontation of emerging problems. Moreover, negative globality was found to be a significant predictor of confidence with a negative beta weight, which means that low levels of negative globality are related to high levels of confidence.

\section{DISCUSSION}

The intercorrelations between the causal dimensions for positive outcomes and the causal dimensions for negative outcomes were positive and statistically non-significant. The lack of negative intercorrelations between the three scales for positive events and the three scales for negative events supports hypothesis 1 . Therefore, high scores on the scales for positive events are not related to low scores on scales for negative events. This finding is in contrast with Seligman's (1990) definition of attributional style in which he suggests that a 
person who attributes successes to internal, stable, and global factors will also attribute failures to external, unstable, and specific causes.

In order to test hypothesis 2 a factor analysis of the six scales was conducted. According to the reformulated model of learned helplessness, three factors are to be extracted, namely, internality, stability, and globality, which correlate with each other. Moreover, the theory implies that the scales for the negative events will load on their intended factor negatively, for example, the scale of negative internality should load negatively on the general factor of internality, while the scale of positive internality will load on the same factor but positively. The results of the factor analysis do not support this theoretical model. The first factor can be called "attributional style for positive events" as it contains the scales of positive internality, positive stability, and positive globality. The second factor consists of negative internality and negative globality, while the third factor holds the scale of negative stability. Consequently, the results of the higher-order factor analysis, as well as the intercorrelation coefficients, indicate that attributional style for positive events and attributional style for negative events should be considered as separate variables.

The alpha coefficients for the dimensions of stability and globality for both positive and negative events were acceptable (Nunnally, 1978), ranging from .65 to .78 . However, the alpha reliabilities of internality for positive as well as for negative events were quite low; that is, .28 for positive internality and .40 for negative internality. The coefficients of alpha reliability for the six scales provide support for hypothesis 3 . The low alphas for the scales of positive and negative internality is an indication of low cross-situational consistency. This is important because high consistency is an essential attribute of any concept of style. On the other hand, the causal dimensions of stability and globality for both positive and negative events do show high coefficients of internal consistency, especially if we take into account the fact that their 
calculation was based on only three or four items. Therefore, since stability and globality seem to demonstrate a quite strong cross-situational consistency, they are interpreted as more reliable measures of the concept of attributional style.

The psychometric issue of the low reliabilities for the internality dimension reported by the current study, as well as by other studies (see table 5.3), needs a theoretical explanation. The small number of items contained in the attributional style questionnaires can be one factor, but why does this primarily effect the alphas for the internality scale? Even in the case of the EASQ (Peterson \& Villanova, 1988), which is an attempt to improve the reliability of the ASQ (Peterson, et al., 1982) by lengthening it to include 24 negative outcomes, internality once again is the least reliable dimension.

A second explanation can be offered within the framework of attributional theory. The emphasis in the early models of attributional reasoning was on people's explanations for specific events, and usually for a single cause of the effect in question (Kelley, 1967). However, social psychologists have proposed that this approach is not appropriate when people are trying to explain complex social phenomena. For these ambiguous and complicated issues it is not surprising that people think that many interrelated causes have an effect on the emergence of social phenomena. Consequently, it has been proposed that ordinary people's knowledge of a domain can be represented as a network of causal intercorrelations or "perceived causal structure" (Kelley, 1983; Antaki, 1985; Livingstone \& Lunt, 1989). Researchers have discussed this "perceived causal structure" by examining the causal explanations given by people for complex social phenomena, for example, unemployment (Furnham, 1982a) and poverty (Furnham, 1982b).

However, it may be that complex social phenomena are not the only stimuli that make people produce complex causal explanations. Single events may also stimulate such perceived 
causal schemata when people are asked to vividly imagine such events happening to them. In the case of the attributional style questionnaires, as respondents are requested to retrieve an event in their memory, they tend to also retrieve many associated factors that led to or caused this event. An indication of the activation of complex causal schemata lies in the fact that respondents frequently complain about having to find the single most likely cause of an event, arguing that a number of causes seem to them to be equally important. As respondents are obliged to restrict themselves to one cause of the event and at the same time perceive a complex causal reality, they have the tendency to come up with causes that will in one event represent attributions about the self (internal) and in another event attributions about others or the situation (external), which are the two main aspects of causality. On the other hand, because choosing a stable or unstable cause and a global or specific one does not determine whether an attribution is biased or self-centred as in the case of internal or external causes, the activation of the complex causal schema does not decrease the internal reliability of stability and globality.

In addition, the suggestion of Miller, Smith, and Uleman (1981), that the distinction of internaVexternal causes can be regarded as reflecting an underlying and more fundamental causality dimension, namely, the chosen/not chosen dimension, can be utilised for explaining the low cross-situational consistency of internality. The proposed dimension of chosen/not chosen refers to the distinction between an act deliberately chosen by the actor, or an outcome caused more deterministically. Therefore, respondents might not be consistent in attributing various events to internal or external factors because they focus on choosing causes which explain events either as the outcome of their own actions or as deterministically caused. However, such an explanation is a mere speculation that requires future research to address it. 
Finally, the dimension of negative globality emerged as a predictor of negative problem orientation in the stepwise multiple regressions where the positive beta weight of .19 was found. It was also a predictor of confidence with a negative beta weight of -.21 . Negative stability failed to enter any of the multiple regression equations. Consequently, the fourth hypothesis was partly confirmed since the causal dimension of negative globality was shown to be a predictor of negative problem orientation and confidence, while stability did not predict either of the independent variables such that its validity as a measure of pessimism and confidence does not seem to be supported. It needs to be mentioned that these are the results of the stepwise method and that the usage of the forced entry method could possibly lead to different results and, therefore, different conclusions.

The findings suggest that attributional style for positive and negative events are distinct variables. They challenge the learned helplessness theory assumption that people who tend to attribute positive events to internal, stable, and global causes have also the tendency to make external, unstable, and specific attributions for negative events. This study demonstrated that workers who gave high scores to one of the dimensions of attributional style for positive events did not simultaneously give low scores to the same dimension for negative outcomes. For example, high scores on positive internality were not related to low scores on negative internality. In addition, the results of this study advocate the omission of internality for both positive and negative outcomes, as low levels of consistency across situations were found. This finding parallels the low Cronbach alphas for the internality dimension reported by several other studies of attributional style (Cutrona, Russell, \& Jones, 1985; Peterson \& Villanova, 1988; Burns \& Seligman, 1989; Furnham, Sadka, \& Brewin, 1992; Furnham, Brewin, \& O'Kelly, 1994; Heaven, 1994). Finally, the scale of negative globality appeared to be a valid measure of pessimism and confidence, while the dimension of negative stability did not appear 
to be related. Consequently, high scores on the causal dimension of negative globality might well be used as an indicator of a generalised tendency to hold low expectations for future success, while scores on negative stability should be interpreted with caution.

Clearly these findings have implications for both the conceptualisation issues regarding attributional style and the actual measurement of the concept. The main purpose of this study was a proposition for a more reliable and valid measure of attributional style. This can be - accomplished by the omission of the dimension of internality since it has constantly proven to be an unreliable measure, the usage of negative events instead of both negative and positive, and finally, the measurement of the dimension of negative globality which was shown to be a valid measure of pessimism and confidence. In conclusion, the findings of this study advocate the use of a measure of attributional style based on the extent to which people hold generalised low expectations for future performance. 


\section{CHAPTER SIX}

A REFORMULATION AND EXTENSION

OF AMABILE'S

CREATIVITY MODEL

BASED ON ATTRIBUTIONAL THEORY 


\section{INTRODUCTION}

This study is an empirical investigation of the proposed attributional reformulation of Amabile's model of creativity. In addition, an extension of Amabile's social psychology of creativity is suggested on the grounds of the identification of social factors that promote creative behaviour via their impact on intrinsic motivation. Within the attributional reformulation of Amabile's model, the effect of attributional style on the formulation of situational attributions is addressed.

\section{A DESCRIPTION OF AMABILE'S MODEL OF CREATIVITY}

Amabile (1983) presented a model of creativity which outlines three major components of individual creativity, as well as the stages of the creative process. The factors that supposedly influence creativity are domain-relevant skills, cognitive skills, and task motivation. Domain-relevant skills consist of the factual knowledge of the domain in question, while cognitive skills refer to a cognitive style that facilitates creativity such as an ability to "break cognitive sets" and to concentrate effort for a long period of time. Task motivation concerns the intrinsic motivation principle of creativity which enhances creative thinking, whereas extrinsic motivation is demonstrably detrimental. According to the theory, a person is intrinsically motivated to engage in an activity if such engagement is viewed as an end in itself and not as a means to some extrinsic goal. Thus, if an individual finds an activity unpleasant or boring, there is no intrinsic motivation; if he/she finds the activity stimulating or fun, intrinsic motivation is present.

Furthermore, Amabile's model of creativity proposes that the process of creative 
thinking consists of the stages of task orientation, task preparation, response generation, and outcome. According to Amabile, the outcome of the creative process has three possible levels: (a) complete attainment of goal (success), (b) no reasonable responses generated (failure), and (c) some progress toward the goal. The model suggests that the outcome of a given run through the process (success, failure, or partial success) can directly influence future task motivation, thereby, setting up a feedback cycle through which future engagement in the same or similar tasks can be affected. If complete success has been achieved, there will be no motivation to undertake exactly the same task but intrinsic motivation for similar tasks will be increased. If complete failure takes place - if no reasonable responses were generated - intrinsic motivation for the task and for similar tasks should decrease. Finally, if partial success has been met, intrinsic motivation will increase when the problem solver has the sense of "getting warmer" in approaching the goal; however, it will decrease when the outcome of the test reveals that the problem solver is essentially no closer to the goal than when he/she begun.

\section{A CRITIQUE OF AMABILE'S MODEL BASED ON ATTRIBUTIONAL THEORY}

This study focuses on the mechanism of the feedback cycle described by Amabile, and more specifically, on the effect of failure on self-reported subsequent task motivation. The model suggests that when a given run through the creativity process leads to failure, motivation for similar tasks will decrease. However, Weiner (1986) has argued that it is the perceived causality of an outcome, specifically causal stability, rather than the outcome itself that determines expectancy shifts and the levels of motivation. If conditions (the presence or absence of causes) are subjectively expected to remain the same, then the outcome experiences on past occasions will be expected to re-occur. A success under these circumstances would 
produce relatively large increments in the anticipation of future success, and a failure would strengthen the belief that there will be subsequent failures. On the other hand, if the causal conditions are perceived as likely to change, then the present outcome will not be expected to repeat itself in the future, or there may be uncertainty about subsequent outcomes. A success would yield relatively small increments, if any, and probably decrements in the expectancy of success, whereas a failure need not necessarily strengthen the belief that there will be future failures.

In the first study of this thesis it was demonstrated that causal stability is a good predictor of after-failure motivation provided that the initial level of motivation to engage in the task is high. Therefore, as the literature on creativity suggests that high motivation and persistence characterise the creative process (Newell, Shaw, \& Simon, 1963; Amabile, 1983), it can be argued that when a person is engaged in a creativity task his/her intrinsic motivation will be affected not by the actual outcome of the process but by the causal attribution which the person undertakes to explain the event. Where failure has been encountered, the intrinsic motivation to engage in similar tasks will be influenced by the person's perception of the causes that led to failure. If failure is attributed to stable, permanent, and unchangeable causes then it can be predicted that expectation for future failure will be enhanced and intrinsic motivation will decrease. On the other hand, if the causes of failure are perceived as unstable, temporary, and changeable then expectancy for subsequent failure will not increase, and may decrease, so that, intrinsic motivation will stay at the same levels or will even get higher.

The attribution of failure in a specific task to stable or unstable causes is to some extent determined by the person's attributional style, as well as by situational information like social comparison, effort expenditure, expectancy of the outcome, and so forth. The examination of the effect of attributional style on the formulation of situational causal stability is important for 
two reasons. Firstly, as attributional style is a result of the accumulated experiences in similar situations leading to habitual patterns of attributions, the investigation of its effect on causal stability enables us to evaluate the influence of past outcome history on situational attributions. Secondly, previous studies (Anderson, 1983; Mikulincer, 1990) have shown that situationally specified attributions are more dominant than attributional style in determining future motivation. However, these studies have neglected the possibility that attributional style might have an indirect effect on motivation through the formulation of situational attributions.

A pessimistic attributional style has been defined recently (Abramson, Metalsky, \& Alloy, 1989) as a tendency to make stable and global attributions across negative situations. In the current study the effects of attributional style on the perception of situational causal stability of failure during a creative process will be examined, and it is predicted that a pessimistic attributional style will be associated with attribution of a specific failure to stable causes.

\section{SOCIAL FACTORS THAT FACILITATE CREATIVE BEHAVIOUR}

In addition, this study addresses the intrinsic motivation hypothesis for creativity which suggests that external constraints, such as evaluation, competition, and surveillance, have a detrimental effect on creativity. Cognitively oriented researchers in the area of creativity have demonstrated that social constraints in task engagement undermine creativity (Amabile, Goldfarb, \& Brackfield, 1990; Amabile, 1982; Amabile, Hennessey, \& Grossman, 1986; Amabile \& Gitomer, 1984). This theoretical approach is mainly based on self-perception theory (Bem, 1972) which argues that people often infer their attitudes from environmental factors that provide cues about their beliefs by using quite similar processes to the ones that they use 
to infer other people's attitudes. Bem's point is that we often infer our attitudes by observing or recalling our own behaviour. Thus, when we are trying to understand how we performed on a specific task we check whether our behaviour was under external control. If it was, then we infer that we do not have intrinsic interest in the task and that it was performed because of external factors. On the other hand, in the case that there are no salient social constraints, it is highly probable that the behaviour will be attributed to intrinsic interest. When creative thinking takes place, the existence of observable or obvious social constraints supposedly leads people to attribute their performance to extrinsic reasons (extrinsic motivation) and not to a genuine interest in the task (intrinsic motivation). According to Amabile's social psychology of creativity, motivational orientation may be the mechanism by which a variety of social factors influence creativity.

However, even though the intrinsic motivation principle of creativity focuses on the negative effects of social factors on creativity, it has been recently acknowledged that future research should examine the circumstances under which social factors might enhance creative thinking (Amabile, Goldfarb, \& Brackfield, 1990). The exploration of the social factors that can be conducive to creativity should be based on the explanation of why some social variables are detrimental to creativity. In other words, if we can identify how social constraints impair creativity, we might be able to find the circumstances under which social facilitation of creative thinking occurs.

One main explanation of the negative effect of social factors on creativity proposed by the cognitive researchers concerns the possible narrowing of attention to goal-relevant stimuli during engagement in externally rewarded tasks. The reduced attention to the intrinsic properties of the task may decrease the flexibility and spontaneity of performance which are important aspects of creative thinking. Eisenbergen and Selbest (1994) have argued that 
explicit reward is detrimental to divergent thinking if presented in a way that attracts increased attention. Increased reward salience, resulting from a greater reward size, proximity, quality and so forth, should reduce the task attention that contributes to creative thinking. In addition, Kasof (1995) considers a nonsalient environment to be conducive to creative production.

Therefore, on the grounds of identifying social factors that do not distract people's attention from the task, it may be argued that it is the construction of a creative atmosphere/climate (Davis, 1986) that might have a positive influence on creativity. Social constraints, such as reward, evaluation, competition, and restricted choice, when presented in a salient way might undermine creativity by narrowing attention to the task. On the other hand, group norms that place a high value on creative thinking might enhance creativity by playing a background role that promotes creativity through the mechanism of causal stability. When a creativity task is undertaken, the outcome can be success, partial success, or failure (Amabile, 1983). Since a negative event is more likely to activate an attributional process, this thesis focuses on the effect of social norms for creativity on intrinsic after-failure motivation via the mechanism of causal stability (see part II of the second chapter). If the group's norms encourage creativity, then the person has the tendency to attribute failure to unstable causes which are potentially changeable. If the social norms discourage creativity and putting new ideas into practice, then it is more probable that the causes of failure will be perceived as more stable and unchangeable. Finally, in the case that the group is indifferent to creativity, then social norms for creativity are not expected to be related to causal stability of failure or levels of subsequent motivation.

The aims of the current study were to test (a) whether failure on a creativity task in itself leads to lower levels of intrinsic motivation, (b) whether self-perceived causal stability in the attribution of failure during a creativity process effects the desire to engage in similar 
tasks, as predicted by Weiner's attributional theory, (c) whether attributional style per se influences the perception of causal stability of situational attributions, and finally, (d) whether social norms for creativity have an effect on the perception of the stability of situational attributions.

In order to avoid the pitfalls of artificial measurement situations designed to elicit creative behaviours this study set out to examine failure in a creative process in a moderately realistic situation. However, if one chooses to examine failure as the result of a creative process the most widely accepted definition of creativity as the activity which leads to a novel, appropriate, and valuable product (Amabile, 1983) is not applicable. This is, mainly because this definition does not allow for failure to be the outcome of a creative process. Therefore, in the case of investigating the effects of failure on intrinsic motivation it is necessary to use a definition of creativity which concentrates on the process. Torrance (1988) has defined creativity "as the process of sensing difficulties, problems, gaps in information, missing elements, something askew; making guesses, and formulating hypotheses about the deficiencies; evaluating and testing these guesses and hypotheses; possibly revising and retesting them; and finally, communicating the results" (p.47).

As the definition of creativity provided by Torrance is almost identical to the process that concerns conducting scientific research, this study examined failure in the context of doing research toward a doctorate. Moreover, since doing a Ph.D. is generally related to high levels of motivation and persistence, causal stability of failure is expected to be a good predictor of after-failure motivation; this is the case because the necessary condition of high initial motivation is fulfilled (see chapter four). Students registered for the doctorate (Ph.D.) degree were asked to remember a specific occasion when they presented a new idea for running an experiment or conducting a study to their supervisor and he/she rejected it. 
Given the above aims the following hypotheses were tested:

Hypothesis 1. Failure in a creativity task per se does not lower subsequent motivation to engage in similar tasks.

Hypothesis 2

Stable attributions for failure lead to low after-failure motivation, while unstable attributions for failure lead to high after-failure motivation.

Hypothesis 3 Low levels of pessimistic attributional style lead to unstable attributions for failure, whereas high levels lead to stable attributions.

Hypothesis 4 Pro-creativity social norms lead to unstable attributions for failure, while anti-creativity social norms lead to stable attributions.

Hypotheses 2, 3, and 4 were tested by conducting a path analysis. In figure 6.1 the relationships between the variables are signified with incoming or outgoing arrows which concern the testing of the above hypotheses.

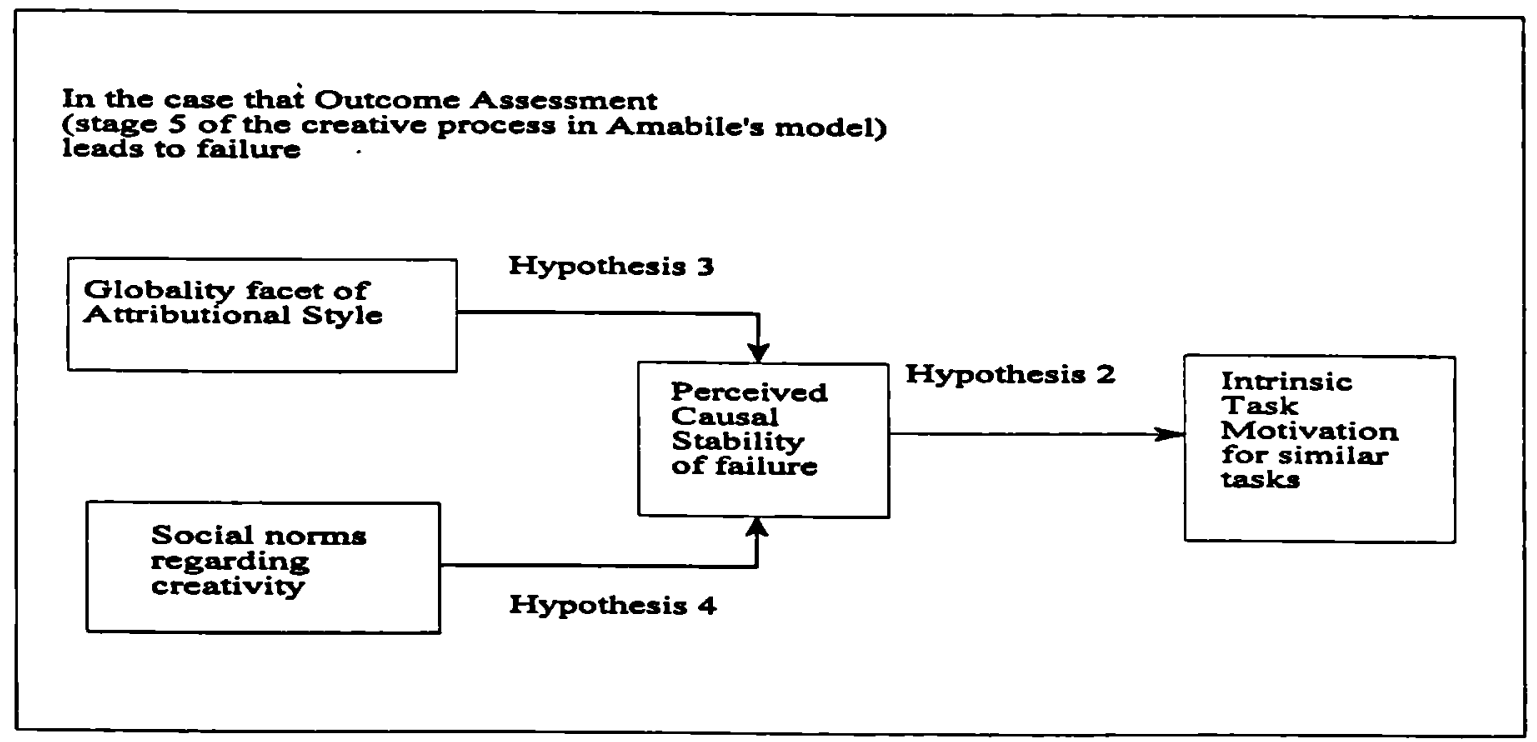

Figure 6.1. Graph showing the hypothesised relations between the model variables. 


\section{METHOD}

Respondents. A total of 66 respondents was recruited primarily at the University of London, University of Wales, University of Surrey, and University of Bristol, coming from a variety of different scientific subjects including engineering, psychology, materials science, computer science, dermatology, physiology, geography. There were $35(53 \%)$ women and 31 (47\%) men. Concerning age differences $47(71 \%)$ subjects were between 20 and 29 years old, 16 (24\%) were between 30 and 39 years old and $3(5 \%)$ subjects were over 40 years old. Regarding the year of their studies, $10(15 \%)$ participants were in the second part of their first year (six months after starting their Ph.Ds), 17 (26\%) in their second year, 27 (41\%) in the third year and $12(18 \%)$ past the third year of their studies. Subjects were asked to rate on two 7-point scales the degree to which firstly, it is important to be creative in their work and secondly, it is important to carry out original experiments/studies. Only 3 subjects (3.2\%) answered that it is not important to be creative and/or to carry out original experiments/studies, who were excluded from any further analysis.

Questionnaires. Subjects were asked to complete a number of questionnaires measuring causal stability of a specific occasion when they presented a new idea for running an experiment (or conducting a study) to their supervisor and he/she rejected it, shift in the intrinsic motivation following the failure event, social norms concerning creativity within their department, the stability and globality dimensions of attributional style, pessimism, and finally, whether the supervisor insists on the originality of their work.

Assessment of causal stability in the case of failure. In order to measure the stability of the 
causes to which failure is attributed, subjects were asked to try to remember one specific occasion when they presented a new idea for running an experiment (or conducting a study) to their supervisor and he/she rejected it. Then the causal stability was measured by using the Revised Causal Dimension Scale's (McAuley, Duncan, \& Russell, 1992; CDSII) subscale of stability in which the subjects have to score the cause of failure on three 7-point scales; the cause being permanent or temporary, stable over time or variable over time, and unchangeable or changeable. The alpha reliability of the subscale of stability was reported to be .67 . McAuley, Duncan, \& Russell (1992) demonstrated the construct validity of the inventory by conducting a confirmatory factor analysis in the LISREL program. In the current study a number of other items from the CDSII subscales of locus of control and locus of causality were used as filler items. The Cronbach alpha obtained in this study was .79 , which is acceptable.

Shift in intrinsic motivation. In the area of creativity research Amabile and her colleagues (1985, 1990) have been assessing intrinsic motivation by asking subjects to fill in a questionnaire containing the following aspects of intrinsic motivation: (a) the subjects' level of interest in the task, (b) the level of enjoyment they get by doing the task, and (c) the degree of satisfaction with the product of their effort. In this study the shift of intrinsic motivation after failing in a creativity task was measured by concentrating on the changes in the levels of how interesting and how challenging the respondents perceived the task to be. A measurement of the levels of enjoyment and satisfaction with the final product were not assessed as they do not seem to apply in the condition of failing. It is highly possible that failure leads to a negative affect because of its nature (Weiner, 1986) but, even though a negative affect is generated, the task can still be perceived as interesting and challenging. The Cronbach alpha for the measurement of intrinsic motivation was .76. 
Social noms concerning creativity. The subscale of Culture Gap Survey (Kilman \& Saxton, 1983; CGS) called "task innovation" was used for assessing social norms about creativity. The constructors of the inventory have reported high levels of internal reliability for the four subscales of the questionnaire (ranging from .83 to .94 ) and demonstrated construct validity by stable four factors solutions across samples. The alpha reliability of the subscale of "task innovation" was reported to be .89 (Saxton, 1987) and .81 (Xenikou \& Furnham, in press; see chapter seven).

Even though the CGS has been developed to assess the cultural norms of groups at the work place, the subscale of task innovation contains items that refer to social norms about creativity and new ideas in such a general way that can be used in assessing social norms of creativity in the university. Moreover, because doing a Ph.D. is a relatively non-social process "task" innovation is a more appropriate measure of norms about creativity than other measures of innovation which concentrate on changes concerning the structure or the strategies of an organisation. Filling in the questionnaire was a forced choice task and the items contained in the subscale are presented in the following table.

Table 6.1. Items that comprise the CGS "Task Innovation" scale.

1. Encourage creativity /Discourage creativity.

2. Make changes /Keep things the same.

3. Try new ways of doing things /Do not "rock the boat".

4. Always try to improve /Do not try to change.

5. Help others put new ideas into practice/Resist put new ideas into practice

6. Be willing to take on new tasks /Resist taking on new tasks.

7. Encourage new ideas/Discourage new ideas.

The Cronbach alpha generated for the CGS subscale of "task innovation" in this study was .66.

The Stability and Globality dimensions of Attributional Style. The measurement of the stability and globality dimensions was modelled on the Expanded Attributional Style Questionnaire 
(Peterson \& Villanova, 1988; EASQ) which has the same instructions and format as the Attributional Style Questionnaire (Peterson et al., 1982; ASQ) except that it contains only negative events rather than both negative and positive. Subjects are presented with 24 hypothetical bad events and in each case, are asked to imagine the event happening to them. They then write the "one major cause of the event" and rate it on a 7-point scale in terms of internality versus externality, stability versus instability, and finally, globality versus specificity. In the current study 10 negative events concerning doing a Ph.D. were generated and subjects had to vividly imagine that these events are happening to them. The hypothetical negative situations are presented in table 6.2.

According to the relevant literature (Cutrona, Russell, \& Jones, 1985; Anderson, Jennings, \& Arnoult, 1988), attributional style should be narrowly specified; that is, it has to contain events that can be classified into the same category (e.g., work-related events, healthrelated events). On the grounds of this suggestion, 10 events were produced concerning the narrowly specified class of situations occurring as part of one's Ph.D. life.

Table 6.2. Hypothetical negative events used to measure negative stability and globality.

1. You are in the situation where your supervisor commented on your literature review as incomplete.

2. You submitted a paper to a conference and it was rejected.

3. The last experiment/study that you carried out failed to provide any (significant) results.

4. You developed a new methodology that was severely criticised by experts in your field.

5. You presented your pilot study in front of other postgrads and departmental staff and there was negative feedback.

6. You can not get all the work done that your supervisor expects of you.

7. Your supervisor rejected your idea for the next experiment/study that you designed.

8. You applied for a grant to attend a course that would be very useful for your research and you did not get it.

9. You had sent a paper to a journal for publishing and the reviewers rejected it.

10. You sent a report to your sponsor about the state of your research and they reply that it seems to go very slowly.

For the purposes of the current study the dimensions of stability and globality were measured, while causal internality was excluded. This was the case mainly because it has been 
already suggested by Xenikou, Furnham, and McCarrey (in press) that the dimension of internality as a component of attributional style has consistently demonstrated low levels of internal reliability which undermines the accurate measurement of the attributional style concept. In the current study the Cronbach alphas for causal stability and globality were .75 and .82 , respectively.

The Hopelessness Scale (Beck, Weissman, Lester, \& Trexler, 1974). The Hopelessness Scale (HS) has been developed in order to measure hopelessness as a system of cognitive schemata whose common denomination is negative expectations about the future. The HS contains 20 items which are scored on a true-false scale. The alpha reliability of the scale is reported by the constructors to be .93 . The concurrent validity of the scale has been demonstrated by moderate correlations with other measures of hopelessness. Indication about the construct validity was provided by showing that depressed patients have a negative attitude about the future, and these negative expectancies are reduced when the patient recovers clinically from his/her depression. The Cronbach alpha of the HS in this study was .87 .

The HS was administrated in the current study in order to test the validity of the measurement of attributional style's causal stability and globality because the hypothetical events of the EASQ were replaced by hypothetical events about specifically doing a doctorate. The obtained correlations between the HS and the causal stability and globality were $.31(\mathrm{p}<$ $.01)$ and $.47(p<.001)$, respectively. Therefore, it is possible to conclude that the measurement of the attributional style's causal stability and globality is shown to be valid, as the correlations with the HS are at a moderate level, statistically significant, and in the predicted direction.

Levels of Originality required by the supervisor. It can be argued that doing a doctorate is not 
necessarily a creative process; for example there are students who are doing a Ph.D. by working on a research project which is already designed or there are supervisors who do not insist on originality. In order to determine whether the event of failure presented to the subjects is actually the outcome of a creative process, the subjects were asked the levels of originality concerning their studies required by their supervisor. The importance of asking this question was demonstrated by the fact that 15 subjects $(25 \%)$ said that the supervisor does not insist on the originality of their work. These subjects were excluded from any further analysis. All the subjects who believed that the supervisor insists on originality scored high on the two items concerning the importance that they themselves place on being creative and original.

Procedure. The participants filled in the questionnaires at their university or at home. They were informed about the study by either reading advertisements like this: "If you have at least six months experience as a Ph.D. student you could help a fellow student with her research and at the same time earn $£ 3$ by completing a questionnaire at home" or by being approached by the researcher who was asking whether they were willing to participate. The questionnaire took between 40 to 45 minutes to complete. The response rate was 90 per cent.

\section{RESULTS}

In order to test whether failing in a creativity task, i.e. having a supervisor reject a proposal for a study, leads to lower levels of intrinsic task motivation a chi-square test was carried out between the group of subjects who scored from 2 to 7 on two 7 -point scales measuring shift in motivation after failure and the group of subjects who scored from 8 to 14 on these scales (low scores indicate low levels of motivation and high scores high levels of 
motivation). A significant chi-square of $\chi^{2}(1)=20.44, p<.001$ showed that there were significantly more subjects in the group that reported the same or higher levels of intrinsic motivation. The observed and the expected frequencies are presented in table 6.3.

Table 6.3. Observed frequencies, expected frequencies, and residuals of shift in motivation measured on two 7-point scales.

\begin{tabular}{lccc}
\hline Category & Observed Cases & Expected Cases & Residual \\
Scores 2-7 & 8 & 23.50 & -15.50 \\
Scores 8-14 & 39 & 23.50 & 15.50 \\
Total & 47 & & \\
\hline
\end{tabular}

Note. Low scores indicate low motivation, while high scores indicate high motivation

To test hypotheses 2,3 , and 4 a path analysis was conducted. The correlations among the five variables that are included in the model and the demographic variables are presented in table 6.4.

Table 6.4. Pearson correlations among causal stability (CS), social norms concerning creativity (SNCC), attributional style's dimension of stability (ASDS), attributional style's dimension of globality (ASDG), intrinsic task motivation (ITM), and the demographic variables (sex, age, and year of studies) .

\begin{tabular}{lllllllll}
\hline & CS & SNCC & ASDS & ASDG & ITM & SEX & AGE & YEARS \\
CS & 1.00 & & & & & & & \\
SNCC & $-.36^{* *}$ & 1.00 & & & & & & \\
ASDS & .04 & $-.39^{* *}$ & 1.00 & & & & & \\
ASDG & $.34^{*}$ & -.19 & $.49^{* * *}$ & 1.00 & & & & \\
ITM & $-.35^{*}$ & -.13 & .18 & -.25 & 1.00 & & & \\
SEX & .16 & -.09 & .00 & .13 & $-.39^{* *}$ & 1.00 & & \\
AGE & .10 & -.02 & -.05 & .16 & .06 & -.21 & 1.00 & \\
YEAR & -.03 & .13 & -.02 & .16 & -.02 & .03 & .25 & 1.00 \\
\hline
\end{tabular}

$\mathrm{N}=47$

${ }^{*} \mathrm{p}<.05 * * \mathrm{p}<.01{ }^{* * *} \mathrm{p}<.001$

Firstly, a hierarchical multiple regression was performed with shift in intrinsic motivation as the dependent variable. This analysis was carried out in two steps. Demographic variables were entered at the first step. By doing so it is possible to examine the effect of causal 
stability, attributional style, and social norms for creativity on shift in motivation after controlling for any effects of the demographics on the dependent variable. At the second step, the independent variables of causal stability, attributional style's facets of stability and globality, as well as the norms for creativity, were entered. This step permitted the examination of whether causal stability, attributional style, and norms for creativity have a direct effect on shift in motivation. The results are presented in table 6.5 .

Table 6.5. Hierarchical multiple regression. Dependent variable: Shift in intrinsic motivation.

\begin{tabular}{llll}
\hline Variable & Beta & $t$ & Sig t \\
\hline
\end{tabular}

Block 1:Demographics

$\mathrm{R}^{2}=.15, \mathrm{~F}(3,43)=2.68$, $\mathrm{p}=.058$

$\begin{array}{llll}\text { Sex } & -.40 & -2.78 & .007 \\ \text { Age } & -.01 & -0.13 & .896 \\ \text { Year of studies } & -.00 & -0.01 & .986\end{array}$

\section{Block 2}

$\mathrm{R}^{2}=.38, \mathrm{~F}(7,39)=3.49$,

$\mathrm{p}<.01$

$\begin{array}{lrrr}\text { Causal Stability } & -.29 & -1.99 & .052 \\ \text { Globality of AS } & -.29 & -1.82 & .076 \\ \text { Stability of AS } & .27 & 1.66 & .103 \\ \text { Norms for Creativity } & -.21 & -1.44 & .155 \\ & & & \\ \Delta R^{2}=.23, F(4,39)=6.06, & & & \\ \mathrm{P}<.01 & & & \end{array}$

$\mathrm{N}=47$

Of the demographic variables, the sex of the respondent had a significant effect on shift in motivation following failure. This association indicated that male respondents report higher levels of motivation after an event of failure compared to females. Above and beyond the effect 
of sex differences on shift in motivation, there is a marginally significant direct effect of causal stability on the dependent variable. Causal stability was found to be a predictor of the criterion with a negative beta weight of -.29 indicating that the attribution of failure to less stable factors causes higher levels of intrinsic motivation.

In the second hierarchical multiple regression the effect of social norms of creativity and attributional style, measured by the dimensions of negative stability and globality, on the perception of causal stability of failure was tested after controlling for the demographic variables. This hierarchical multiple regression was used to test hypotheses 3 and 4 . The three predictors accounted for a quarter of the variance.

Table 6.6. Hierarchical multiple regression. Dependent variable: Causal stability of failure.

\begin{tabular}{llll}
\hline Variable & Beta & $t$ & Sig t \\
\hline Block 1: Demographics & & & \\
$\mathrm{R}^{2}=.05, \mathrm{~F}(3,43)=0.81$, n.s. & & & .185 \\
Sex & .20 & 1.34 & .293 \\
Age & .16 & 1.06 & .602 \\
Year of studies & -.08 & -0.52 & \\
& & & \\
Block 2 & & & \\
$\mathrm{R}^{2}=.28, \mathrm{~F}(6,40)=2.61$, & & & .018 \\
$\mathrm{p}<.05$ & & & .014 \\
& & -2.46 & .082 \\
Norms for Creativity & -.36 & 2.54 & \\
Globality of AS & .41 & -1.78 & \\
Stability of AS & -.29 & & \\
$\Delta \mathrm{R}^{2}=.23, \mathrm{~F}(3,40)=4.25$, & & \\
$\mathrm{P}<.05$ & & & \\
\hline N=47 & & & \\
\end{tabular}

The results show that none of the demographic variables influence causal stability of failure. Moreover, norms for creativity, as well as attributional style's facet of globality, had a significant effect on the perception of causal stability. That is, the existence of pro-creativity 
social norms and/or an attributional style of making specific (not generalised across situations) attributions for negative events lead to the perception of the causes of failure as unstable and changeable.

Since attributional style's negative stability was not shown to affect causal stability a third multiple regression was conducted after excluding the variable of attributional style's facet of stability. Causal stability for failure was regressed on attributional style's dimension of globality and social norms concerning creativity, and accounted for a fifth of the variance $\left(R^{2}=\right.$ $.20)$.

Table 6.7. Regression of causal stability of failure on social norms for creativity and negative globality.

\begin{tabular}{lrrr}
\hline Variable & Beta & $\mathrm{t}$ & Sig t \\
\hline & & & \\
Norms for Creativity & -.29 & -2.15 & .036 \\
Globality of AS & .28 & 2.11 & .040 \\
$\mathrm{R}^{2}=.20, \mathrm{~F}(2,44)=5.61$, & & & \\
$\mathrm{p}<.01$ & & & \\
& & & \\
\hline
\end{tabular}

$\mathrm{N}=47$

The results show a positive association between attributional style's globality and the attribution of failure to stable factors. Therefore, people who have the tendency to attribute negative events to causes that have a general effect on many areas of their lives provide more stable causes in their attempt to explain an event of failure. On the other hand, people with a low score on pessimism tend to perceive the causes of failure as less permanent. In addition, as norms for creativity were found to be a predictor of causal stability of failure with a negative beta weight of $-.29(\mathrm{p}=.036)$, it may be suggested that social groups which promote creativity lead their members to attribute failure to unstable and changeable causes. 
The combined effect of norms concerning creativity and attributional style's dimension of globality is statistically significant $(F(2,44)=5.61, p=.01)$ indicating that when the social environment holds pro-creativity norms and the members of this group do not have the predisposition to attribute negative events to global factors, then less stable causes of failure are expected to be provided, after-failure intrinsic motivation is higher, and thus, creativity is possibly enhanced.

In addition, a second path analysis was carried out in which the effect of the demographic variables was not controlled. In the first multiple regression shift in intrinsic motivation was regressed on causal stability for failure, attributional style's facet of stability, attributional style's facet of globality, and finally, social norms for creativity. A multiple correlation coefficient of $R^{2}=.27, F(4,42)=3.90, p=.008$ was found.

Table 6.8. Multiple regression. Dependent variable: Shift in intrinsic motivation.

\begin{tabular}{lrrr}
\hline Variable & Beta & $\mathbf{t}$ & Sig t \\
\hline Causal Stability & -.33 & -2.15 & .037 \\
Globality of AS & -.30 & -1.86 & .069 \\
Stability of AS & .27 & 1.66 & .103 \\
Norms for Creativity & -.19 & -1.26 & .212 \\
& & & \\
$\mathrm{R}^{2}=.27, \mathrm{~F}(4,42)=3.90$, & & & \\
$\mathrm{p}<.01$ & & &
\end{tabular}

$\mathrm{N}=47$

The results show that causal stability of failure affects the shift in intrinsic motivation to engage in similar tasks. The effect of causal stability on the criterion reached significance ( $p=.037)$, while its effect on the criterion was marginally significant when controlling for the demographic variables. 
In the second multiple regression causal stability was regressed on attributional style's facets of stability and globality, as well as norms for creativity, without controlling for the demographic variables. A multiple correlation coefficient of $\mathrm{R}^{2}=.26$ emerged.

Table 6.9. Multiple regression. Dependent variable: Causal stability of failure.

\begin{tabular}{lccc}
\hline Variable & Beta & $\mathrm{t}$ & Sig t \\
\hline Norms for Creativity & -.38 & -2.74 & .008 \\
Globality of AS & .42 & 2.84 & .006 \\
Stability of AS & -.31 & -1.96 & .055 \\
$\mathrm{R}^{2}=.26, \mathrm{~F}(3,43)=5.28$, & & & \\
$\mathrm{p}<.01$ & & & \\
& & & \\
$\mathrm{~N}=47$ & &
\end{tabular}

Norms for creativity and the globality facet of attributional style were shown to influence the causal stability of failure. Pro-creativity social norms and a tendency to attribute negative events to specific causes lead to the perception of the causes of failure as unstable. Finally, attributional style's facet of stability had a marginally significant effect on causal stability with a negative beta weight of -.31 . The negative beta weight means that the higher the person's disposition to make stable attributions for negative events the less stable attributions he/she would provide on the specific occasion of the rejection of his/her idea for a new experiment/study. These results might be an artifact of the regression model rather than indicating that there is a negative relation between situational causal stability and the attributional style's facet of stability since the row correlation is small, but positive $(r=.04)$.

Figure 6.2 shows the model of the effect of failure on shift in intrinsic motivation when the demographic variables are omitted. Concerning the effect of social norms for creativity and attributional style's facet of globality on causal stability, the beta weights of the regression 
In the case that Outcome Assessment

(stage 5 of the creative process in Amabile's model)

leads to failure

$.28 *$

Globality facet of Attributional Style

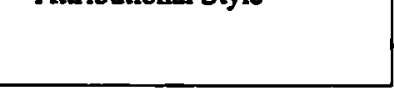

regarding

creativity

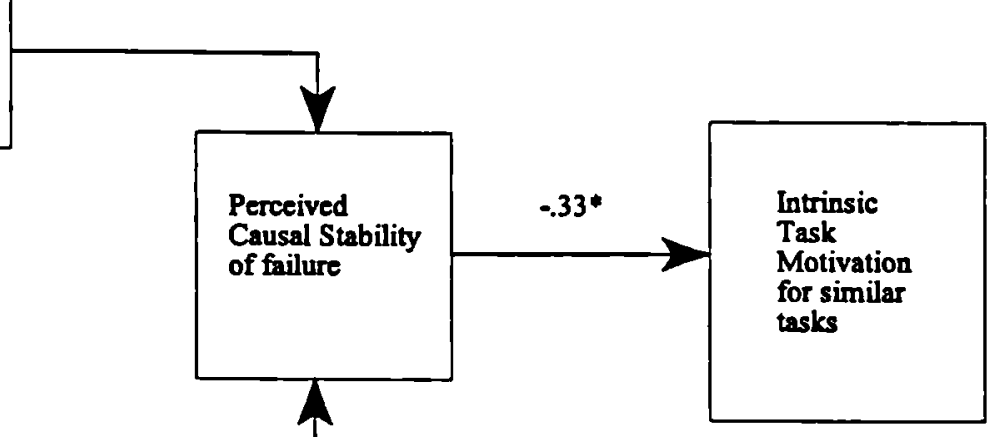

$-.29 *$

$* \mathrm{P}<.05$

Figure 6.2. A model of the effect of failure in a creativity task on intrinsic task motivation.

presented in table 6.7 were used instead of the beta weights of the regression presented in table 6.9. This is the case because although both of the regression analyses do not control for the demographic variables, the regression presented in table 6.7 does not include a predictor which fails to reach significance.

The model shows that there is a significant direct effect of causal stability of failure on shift in intrinsic task motivation. That is, more stable situational attributions of failure lead to lower levels of motivation, while more unstable attributions lead to higher after-failure 
motivation. In addition, attributional style's dimension of globality and social norms for creativity have an indirect effect on shift in motivation via their impact on causal stability. A tendency to make specific attributions for negative events leads to unstable situational attributions of failure, and therefore, to higher levels of after-failure motivation. Moreover, a pro-creativity environment causes the attribution of failure to less stable factors which leads to higher levels of intrinsic motivation.

Finally, the hypothesised model was assessed using Linear Structural Relationships (LISREL) VIII program (Jöreskog \& Sörbom, 1993). Structural equation modelling is particularly useful in that it directly deals with the possible unreliability of scales used in psychological research. That is, the LISREL program enables us to test for the hypothesised relations between psychological constructs after taking into account the reliability of the constructs' measurement.

The LISREL program provides a chi-square test of the overall goodness-of-fit of the model, or the extent to which the hypothesised model is able to account for the observed relations among the variables. In other words, the chi-square goodness-of-fit test assesses the adequacy of the hypothesised model in terms of its ability to re-create the observed covariance matrix. Models that result in a predicted covariance matrix that significantly deviates from the observed covariance matrix are judged to be inadequate. Therefore, a statistically significant value of the chi-square test indicates that the hypothesised model cannot account for the correlations among the observed variables.

One problem that arises in using the chi-square test in evaluating the fit of hypothesised models to empirical data is that the test is sensitive to sample size. In the case of studies employing large samples, it is possible that the chi-square test can lead to models being rejected (resulting in a significant chi-square value) when the models fit the data quite well. 
However, in the current study a rather small sample size was employed, and therefore the chisquare goodness-of-fit can be used for the evaluation of the hypothesised model.

The model that was tested hypothesised that norms concerning creativity, as well as a pessimistic attributional style effect the perceived stability of the attributions for failure which, in turn, influences the levels of after-failure motivation to engage in similar tasks.

The reliability coefficients of the scales reported by previous research were used in order to test for the relations between the variables after correcting for any measurement error. For the scale of norms concerning creativity, an alpha reliability of .81 (Xenikou \& Furnham, in press) and a test-retest reliability of .89 (Saxton, 1987) were reported; therefore, an average reliability of .85 was calculated. Alpha reliabilities of $.62, .54, .60, .88, .55, .69, .75, .60, .67$ (Cutrona, Russell, \& Jones, 1985; Peterson \& Villanova, 1988; Furnham, Sadka, \& Brewin, 1992; Heaven, 1994; Furnham, Brewin, \& O' Kelly, 1994; Hull \& Mendolia, 1991; Xenikou, Furnham, \& McCarrey, in press) were reported for the attributional style's scale of negative globality; an average reliability of .65 was calculated. Finally, an alpha coefficient of .67 (McAuley, Duncan, \& Russell, 1992) was reported for the scale of causal stability. There are no coefficients of reliability for the scale used in the current study to measure shift in motivation after failure, thus the latent variable could not be estimated.

The square root of the reliabilities (or average reliabilities) for each scale were computed which is the loading of the observed variable on its latent construct. The measurement error was calculated by subtraction ( 1.00 minus reported reliability). For example, for the scale measuring causal stability the reported reliability coefficient was utilised to calculate, firstly the loading of the observed variable of causal stability on its latent construct (the loading is the square root of the reliability coefficient .67 ), and secondly the error variance which is calculated by subtracting the reliability coefficient .67 from the reliability coefficient 
which indicates that there is no measurement error, that is, the value of 1.00 .

The model is shown in figure 6.3. A non-significant chi-square, $\chi^{2}(3, N=47)=7, \mathrm{p}$ $=.072$, was computed for the model indicating that the model provided a good fit to the data. The labelled large circles represent the latent (unmeasured) variables and the rectangles represent observed (or measured) variables. The single headed arrows from the large circle to the rectangles indicate that the observed variables are theorised to be generated by the latent construct. The values within the small circles represent the unique portion of residual variance (measurement error) that is not accounted for by the latent variable. Finally, the arrows connecting the large circles represent the relations between the latent variables; that is, the 
relations between psychological constructs after removing the possible effect of measurement error.

Since the significance level of the chi-square value is above .05 , the theorised model fitted the empirical data after correcting for any measurement error. Therefore, the results show that causal stability is a predictor of shift in motivation with a negative beta weight of -.32. Moreover, attributional style's facet of globality and social norms for creativity affect the formulation of causal stability of a specific failure.

In the Lisrel model the relationship between the variables was tested without controlling for the demographics. Therefore, if one compares the beta weights of the Lisrel model to the beta weights of the path analysis model which did not include the effect of the demographic variables, it is possible to suggest that when the measurement error of the scales was taken into account the beta weights can either be higher or lower.

\section{DISCUSSION}

This study set out to examine whether failure in a task that requires creativity causes lower levels of intrinsic motivation to engage in similar tasks, as Amabile's (1983) model of creativity suggests. It is argued that attributions for failure is the variable that determines the levels of subsequent motivation for similar tasks rather than failure per se. The findings indicate that failing in a creativity task does not necessarily cause less intrinsic task motivation, while the attribution of failure to stable or unstable factors affects the levels of intrinsic motivation. More specifically, attribution of failure to unstable causes leads to higher levels of intrinsic motivation, whereas intrinsic motivation decreases when failure is attributed to stable factors. These findings support Weiner's (1986) theory of achievement motivation and emotion which 
proposes that motivation depends mainly on the attribution of failure rather than failure itself.

The path analysis controlling for the demographic variables provided beta weights and levels of significance that are lower compared to the ones emerging from the path analysis in which the effect of the demographics was not controlled. Causal stability was shown to have a significant negative effect on after-failure motivation in the case that the analysis did not control for the demographic variables, while there was a marginally significant negative effect when the demographic variables were controlled. Finally, the Lisrel model shows that the strength of the relation between the variables changed when the measurement error was taken into account.

Furthermore, attributional style's negative globality as a measure of pessimism was found to influence causal stability of failure. When people have the tendency to attribute negative events to specific causes, for example attributing failure at maths to low maths aptitude (specific cause) rather than low intelligence (global cause), then it is more likely that failure will be attributed to unstable reasons and that intrinsic motivation will stay at the same levels or will even increase. On the other hand, if people are pessimists (attributing negative outcomes to global factors), failure in a creativity task will be attributed to stable causes and intrinsic motivation to perform similar tasks in the future will decrease.

The effect of the attributional style's dimension of globality on the formulation of the stability of situational attributions provides some evidence that the relationship of the two variables can be more complex than has already been suggested in the relevant literature (Anderson, 1983; Mikulincer, 1990). Even though previous studies demonstrated that situational attributions are more dominant than attributional style on determining the levels of after-failure motivation, it was shown here that attributional style's globality can effect the perception of the stability of situational attributions, which, in turn, determines future 
motivation. Therefore, the effect of attributional style on motivation is not eliminated by the presence of situational attributions but rather attributional style affects future motivation via its impact on the perception of situational attributions.

It should be pointed out that there was a marginally significant negative association between attributional style's dimension of stability and causal stability of a specific failure in the model that did not control for the demographics. This means that a person who has the tendency to choose stable factors in explaining negative events is more likely to attribute a specific occasion of failing to unstable factors. This paradoxical finding is possibly an artifact of the regression model (since the row correlation between the two variables is small but positive), or might concern the issue of the validity of attributional style's dimension of stability. The issue of the validity of attributional style's facet of stability may in part be due to the different format of the items measuring attributional style's stability in the ASQ (Peterson et al., 1982) and the EASQ (Peterson \& Villanova, 1988). In the ASQ each of the negative events for which the respondents have to provide the major cause are reiterated when the item of the stability of the cause is presented, for example when the event is that "you have been looking for a job unsuccessfully for some time" the stability item is "in the future when looking for a job, will this cause again influence what happens?" On the other hand, the EASQ provides the negative event, asks for the major cause of the event and then presents the stability dimension item which does not repeat the event, for example the event is that "you have been looking for a job unsuccessfully for some time" the stability item is "in the future, will this cause again be present?".

Even though there has not been any reported difference between the two ways of measuring the dimension of stability in the relevant literature, it is possible that there is. When participants are responding to the ASQ format they are asked if the cause will influence again 
what will happen when the same negative event occurs, while when they respond to the EASQ format the question is whether the cause will again influence what happens. Therefore, it might be the case that the ASQ format provides information about the stability of a cause in producing a specific negative event which the participant might believe that does not happen often. For example, if the negative event is that the supervisor commented on the literature review as incomplete and the provided reason is the lack of time, then the participant might give a high score on the stability of the cause because he/she believes that every time that the literature review is incomplete it is highly possible that this will occur as a result of lack of sufficient time. However, the respondent might additionally believe that there are not many chances that this negative event will keep taking place. Consequently, it is advisable that the EASQ item format is used instead of the ASQ format.

Finally, social norms for creativity were found to effect the attribution of failure to stable or unstable causes. The relationship between social norms for creativity and causal stability can be explained on the basis of the effect of social comparison. Social comparison literature suggests that the experience of a threatening event (for example, failure) leads to a need to compare oneself with less advantaged others (Wood, 1989; Levine \& Green, 1984) or at least equals on the dimension under evaluation. Groups that promote creative behaviour encourage their members to try new ways of doing things and therefore, it is probable that failures are quite frequently encountered since changing things cannot always be successful. Thus, the members of these groups are likely to compare their failure with similar incidents happening to their colleagues which leads to the perception of failure as caused by changeable causes. On the other hand, groups that have a philosophy of keeping things the same do not accept failure on any grounds, since what is important is failure to be avoided rather than innovation to take place. Under these circumstances, if members dare to propose a new idea 
and this idea fails to be accepted or implemented, then they will probably attribute this failure to stable factors since they cannot compare it to the failures of others. Thus, when the threatening event of failure is encountered the member of the pro-creativity group can more easily find comparison targets (other members who failed in the past) compared to the member of the anti-creativity group leading to the perception of the causes of failure in a different way.

This study was an attempt to examine the effects of failure in a creativity task, as well as the effects of the attributions concerning this failure. It can be concluded that failing is not a sufficient cause for intrinsic motivation to decrease and thus, for creativity to be undermined. The attribution of failure to stable or unstable causes is important in predicting intrinsic motivation and perhaps future intentions to engage in similar tasks. Furthermore, it was demonstrated that low levels of pessimism, as well as pro-creativity social norms, have an indirect positive effect on after-failure motivation via their impact on causal stability.

On the grounds of the findings of this study a proposition for the reformulation and extension of Amabile's model of creativity is put forward. In the case that the outcome of the creative process is failure, the model should incorporate the finding that the effect of failure on subsequent motivation depends on the causal stability of the attributions. Failure per se is not related to lower levels of after-failure motivation. It is rather the stability of the situational attributions that determine the fluctuation of motivation to engage in similar tasks. Provided that the initial motivation to carry out the task is high, failure has a detrimental effect on future motivation if stable causes are used for explaining why failure occurred. In addition, an extension of the social psychology of creativity is proposed based on the identification of social factors that enhance intrinsic motivation to be creative. Pro-creativity social norms were found to have an indirect positive effect on after-failure motivation to engage in creativity tasks via their impact on the causal stability of attributions for failure. 
In conclusion, the findings of the current study indicate that Amabile's model of creativity has to be reformulated in order to incorporate attributions as the moderator of the relation between failure and subsequent motivation. Furthermore, an extension of Amabile's social psychology of creativity is suggested, which concerns the identification of pro-creativity social norms as a facilitator of creative behaviour. 


\section{CHAPTER SEVEN}

A CORRELATIONAL AND FACTOR ANALYTIC

STUDY OF FOUR QUESTIONNAIRE MEASURES OF ORGANISATIONAL CULTURE 


\section{INTRODUCTION}

Before setting out to examine the effect of organisational culture, as a set of norms and values underlying human activity within organisations, on employees motivation to innovate, an important preliminary task was the identification of a reliable and valid measure of organisational culture. The importance of this task lies mainly in the fact that there is a debate regarding the usage of quantitative or qualitative methods in the study of organisational culture. A review of the literature revealed four major self-report measures of organisational culture measuring organisational behavioural norms or values. This study examines the psychometric properties of the questionnaires, and more specifically their internal reliability and convergent validity. In addition, a factor analysis was conducted to investigate whether the various dimensions of culture measured by the four questionnaires could provide a conceptual model of the dimensions of organisational culture. In particular, the factor analysis was used in order to explore whether the dimensions of organisational culture reported in the literature to promote innovation (e.g., a culture that values creativity, achievement, and internal co-operation), or inhibit it (e.g., centralisation of power) would cluster together.

\section{THE ISSUE OF THE RESEARCH METHODS IN THE STUDY OF ORGANISATIONAL CULTURE}

The growth in interest in the concept of corporate culture in the late 1970's and 1980 's led naturally to the development of different self-report questionnaires to measure 
it. However, most of the early studies of organisational culture have relied almost exclusively on qualitative methods as is clearly demonstrated by Glick (1985) who attempted to clarify the differences between the concepts of organisational culture and climate. According to Glick, one of the two things that distinguish culture from climate is that "climate research tends to be nomothetic, using quantitative techniques to describe phenomena while culture research is primarily idiographic, employing qualitative methods to explain dynamic processes" (p. 612).

Advocates of qualitative methods have provided two main justifications for their choice. The first one is based on the presumed inaccessibility, depth or unconscious quality of culture. For example, Schein $(1984,1985,1986)$ suggests that the most important level of organisational culture is named "basic assumptions." Basic assumptions exist at a preconscious level and can be traced through a complex interactive process of joint inquiry between insiders and outsiders. Furthermore, he argues that quantitative assessment conducted through surveys is unwise because it reflects conceptual categories not the respondent's own, presuming unwarranted generalizability. The second point concerns the possible uniqueness of an organisation's culture such that an outsider cannot form a priori questions or measures. This reflects the old emic/etic distinction and dilemma found in cross-cultural psychology (Furnham \& Bochner, 1986). Smircich (1982), on the other hand, conceptualised organisational culture as a particular set of meanings that provides a group with a distinctive character, which in turn leads to the formulation of a social reality unique to members of a group, and as such, makes it impossible for standardised measures to tap cultural processes.

There are good reasons for using qualitative methods in investigating organisational 
culture, but the advantages may be bought at a cost as the data collected usually cannot be the basis for systematic comparisons (Siehl \& Martin, 1988). Fundamental theoretical aspects of the concept of organisational culture can be tested only by comparisons across organisations or/and organisational departments. For instance, the theoretical assumption that the consensus of organisational members on a set of cognitions and practices is the core aspect of culture might be tested by comparing the individual responses of members and the extent of their communality. Moreover, in order to examine if an organisation has subcultures with distinctive values/practices, data can be collected from different departments/units of the same organisation so that within-organisation comparisons might provide us with some information about the existence of departmental or unit cultures. These two and other central theoretical questions cannot be answered until culture can be measured with the same robust, reliable, sensitive, and valid instrument that allows systematic comparisons. Systematic comparisons are exceedingly difficult to make, when only qualitative data are available. Furthermore, some qualitative data are non-parametric precluding any multivariate analysis of the data which almost always require it.

Rousseau (1990) argues that different research methods should be used in the organisational culture research depending on the element of culture to be examined:

Starting at the point of greatest subjectivity, assumptions unconsciously held are difficult to assess without interactive probing. Members fears and defenses are elusive psychodynamics difficult to elicit without interaction. In contrast, characteristic patterns of behaviors (norms) regarding how members should (or should not) act are far more accessible. The method appropriate to assessing culture depends on those elements we choose to examine. As the elements of culture we are interested become more conscious (values, behavioral norms) or observable (artifacts), these are accessible by both structural and nonstandardized assessments. (p. 166)

There are a number of studies in organisational culture that have combined quantitative and qualitative approaches in investigating cultural phenomena. For example, 
Siehl and Martin (1988) studied socialisation of new employees by using what they call "a hybrid measure of culture." Their method consists of two phases. In the first stage, in-depth interviews, ethnographic observation, and archival data help to gain an understanding of the content of a culture. In the second stage, these qualitative data are used to construct a questionnaire, responses to which can be coded quantitatively. Moreover, Hofstede, Neuijen, Ohayv, and Sanders (1990) examined the culture of ten organisations by conducting in:depth, open-ended interviews in order to enrich an existing questionnaire, which could be used for statistical comparisons over organisations and over time.

\section{QUANTITATIVE MEASURES OF ORGANISATIONAL CULTURE}

The available measures of organisational culture concentrate on behavioural norms or values. Rousseau (1990) suggests that organisational culture has a number of layers, two of which are behavioural norms (the way people should behave) and organisational values (the things that are highly valued) and that these layers are characterised by a core theme. As a consequence, some corporate culture test constructors have focused on behaviours, the others on values. Based on this theoretical construct suggested by the culture literature, two questionnaires were used in this study that intend to measure behavioural norms as expectations regarding how members should behave and interact with others, while two others were concerned about values as priorities or preferences.

Measures of behavioural norms include the Organisational Culture Inventory (OCI) developed by Cooke and Lafferty (1989) and the Culture Gap Survey by Kilman and Saxton (1983), which according to Rousseau (1990) show a fair amount of overlap in the 
dimensions used to assess organisational culture. The task-people distinction appears in the conceptual models developed by both Cooke and Lafferty (1989) and Kilman and Saxton (1983). The OCI's subscales labelled "task orientation" and "people orientation" and the CGS's subscales named "technical concern" and "human concern" attempt to measure the same theoretical construct. The second dimension in each of the two models also seem to resemble each other. Cooke and Lafferty (1989) refer to this dimension as security vs. satisfaction needs while Kilman and Saxton (1983) name it as short term orientation vs. long term orientation. In both inventories, this dimension concerns the tendency observed in an organisation either to encourage members to avoid conflict and protect themselves or to innovate and take risks. Consequently, this dimension refers to norms which can be characterised as behaviour inhibiting or behaviour enhancing and it can be labelled as resistance to change-openness to change (Rousseau, 1990). Corporate values can be assessed in the Organisational Beliefs Questionnaire (OBQ) developed by Sashkin (1991) and the Corporate Culture Survey (CCS) by Glaser (1983). As far as their content is concerned there appears to be little overlap traced between the subscales of these questionnaires.

\section{AIM AND HYPOTHESES OF THE CURRENT STUDY}

The aim of this study is to focus on the quantitative assessment of culture by comparing and contrasting these four rather different, but supposedly equivalent, questionnaire measures of culture. Rousseau (1990) in a comprehensive review found seven scale measures of organisational culture measuring either behavioural norms or corporate 
values. The four questionnaires used in the current study are considered by Rousseau (1990) to be among the most established measures of organisational culture. Since most scales have over 50 items the respondents were asked to complete only four scales so that they would not be overloaded. Some of the questionnaires of organisational culture that were not included in this study are the Organisational Culture Profile (O'Reilly, Chatman, \& Caldwell, 1988), and the Norms Diagnostic Index (Allen \& Dyer, 1980). The Organisational Culture Profile items are sorted into nine categories via a Q-sort technique, so it is not directly comparable with the rest of the culture questionnaires which use Likert point scales. Finally, according to Rousseau's review the Norms Diagnostic Index resembles a climate survey.

First, a correlational analysis was conducted on the inventories' subscales which intend to measure the same dimension of culture in order to test the convergent validity of the questionnaire measures. Based on the fact that the description of organisational culture is conceptualised and operationalised quite differently by the four questionnaires, it would be problematic to validate each other by correlating the total scores on each inventory. Consequently, the convergent validity of the questionnaire measures was examined by conducting a correlational analysis on the inventories' subscales which intend to measure the same dimension of culture.

The hypothesis regarding the similarity of the subscales was based on the description of each subscale's content as it is provided by the various models used for the development of the questionnaires, the face validity of the items, and finally Rousseau's (1990) suggestion regarding the theoretical similarity of the OCI and the CGS subscales. More specifically, the observed overlaps among the OCI and the CGS mentioned previously were 
tested so that it would be possible to see whether the respondents perceive the items comprising the overlapping subscales as similar to each other. Moreover, based on the taskpeople distinction and the resistance to change-openness to change one, a number of overlaps were traced among the OBQ and the two behavioural norms questionnaires. The OBQ subscale measuring innovation appears to measure the same cultural dimension as the OCI subscale measuring satisfaction needs and the CGS subscale measuring long-term orientation. The final set of overlaps was traced among the OBQ subscale labelled "The Value of People" and the OCI and the CGS subscales labelled "People Orientation" and "Human Concern," respectively. Finally, the CCS did not appear to measure any cultural dimensions similar to the dimensions measured by the other inventories.

Hypothesis 1. There are high correlations among the overlapping subscales of the four inventories as presented in Table 7.1.

Table 7.1. Predicted Correlations among overlapping Subscales of OCI, CGS, and OBQ.

\begin{tabular}{|l|l|l|}
\hline Scale of OCI & $\begin{array}{l}\text { predicted to be } \\
\text { correlated with }\end{array}$ & Scale of CGS \\
\hline Task Orientation & & Technical Concern \\
\hline People Orientation & & Human Concern \\
\hline Security Needs & & Short-term Orientation \\
\hline Satisfaction Needs & & Long-term Orientation \\
\hline Scale of OCI & & Scale of OBQ \\
\hline People Orientation & & The Value of people \\
\hline Satisfaction Needs & & Innovation \\
\hline Scale of CGS & & Scale of OBQ \\
\hline Human Concern & & The Value of People \\
\hline Long-term Orientation & & Innovation \\
\hline
\end{tabular}


Second, a factor analysis was carried out in order to investigate whether the various dimensions of culture measured by the four questionnaires could provide a conceptual model of the dimensions of organisational culture. Just as there is a lengthy and acrimonious debate in the personality literature on the basic fundamental dimensions of personality and how they are related, so with the growth of corporate culture questionnaires the user is offered different ways of measuring different dimensions. The question remains as to the relationship between these different dimensions measured by different questionnaires.

Hypothesis 2. A clearly interpretable factor structure would emerge from a higher-order factor analysis of the 30 subscales scores from the four different measures.

\section{METHOD}

Respondents. The sample of this study consisted of respondents who were employees in two British organisations. For reasons of confidentiality the names of the companies will not be revealed and we will be referring to them either as organisation A (OA) or organisation B (OB). OA is involved in the domain of publishing/mass communication, while $O B$ is in the cosmetics industry. In all, 157 respondents took part in the study, 79 being employees of $\mathrm{OA}$ and 78 being employed by OB. They ranged in age from 20 to 69 years (the mean being 38 years, $\mathrm{SD}=11$ years). As regards their hierarchical level, $87 \%$ of members in $\mathrm{OA}$ occupied managerial positions and $13 \%$ respondents had non-managerial positions, while in $\mathrm{OB}$ all the respondents held managerial positions (line, middle, and senior management). 
Finally, as far as the years of seniority are concerned, $9 \%$ of the respondents in OA had been with the organisation less than 1 year, 38\% from 1 year to 6 years and 53\% over 6 years. In OB 28\% were employed from 1 to 6 years and 72\% over 6 years. The respondents of OA answered three of the questionnaires, namely the OCI, the CGS, and the $\mathrm{OBQ}$, while the respondents of $\mathrm{OB}$ completed all the inventories. The sample was biased toward a white-collar, better educated group, but there is no reason to believe that this should affect the analyses performed here. The two samples were combined because the analyses of this study focus on within-subject differences rather than between-group comparisons.

Questionnaires. Subjects were required to complete four organisational culture questionnaires which meant a total of 218 questions. The order of the questionnaires was randomised.

Organisational Culture Inventory (Cooke \& Lafferty, 1989). The OCI focuses on behaviours that facilitate fitting in to the organisation and meeting expectations of co-workers. It consists of 12 basic subscales which are the following: Humanistic/Helpful, Affiliation, Achievement, Self-Actualization, Approval, Conventionality, Dependence, Avoidance, Oppositional, Power, Competitive, Perfectionism. These subscales reflect a model based on the intersection of two dimensions which are task-people and security-satisfaction, and which provide the four secondary subscales of the questionnaire. There are 120 items, each one rated on a 1 to 5 Likert scale. The Cronbach's alpha coefficient of internal reliability has been reported to range from .67 to .92 (Cooke \& Rousseau, 1988). As far as the 
validity of the measure is concerned, there have been moderately high levels of withinorganisation agreement on OCI responses and stable factor solution across samples (Cooke \& Rousseau, 1988).

Culture Gap Survey (Kilman \& Saxton, 1983). The CGS was developed to measure behavioural norms. There are four subscales reflecting a $2 \times 2$ framework (Technical/Human Concern and Short/Long-term Orientation): Task support, Task innovation, Social relations, and Personal freedom. Test-retest reliabilities (1 month) ranged from .83 to .94 . Construct validity was demonstrated by stable four factors solutions across samples (Saxton, 1987), while weak relations with group and organisational morals (Saxton, 1987) provide some information about the criterion-related validity.

Organisational Beliefs Questionnaire (Sashkin, 1991). This is a 50-item questionnaire with 5-point Likert scales (strongly agree to strongly disagree) measuring organisational values. The inventory has ten subscales: Work should be fun, Being the best, Innovation, Attention to detail, Worth and value of people, Quality, Communicating to get the job done, Growth/profit/other indicators of success, Hands-on management, and the importance of a Shared philosophy. The 50 items were chosen to minimize social desirability: for each subscale one item is stated positively and the other negatively and the wording is constructed to make it difficult to determine item's desirability. Consensual validity is demonstrated by relatively low within organisation variance in responses.

Corporate Culture Survey (Glaser, 1983). The development of this questionnaire is based 
on Deal and Kennedy's (1982) description of culture types and intends to measure organisational values. It consists of 20 items rated on a 5-point Likert scale, from 5 (strongly agree) to 1 (strongly disagree). The questionnaire holds four subscales which are the following: Values, Heroes/heroines, Traditions/rituals, and finally Cultural network. There are no reported coefficients of reliability or any known demonstration of the questionnaire's validity.

Procedure. The respondents filled in the questionnaires at their place of work in groups or during their spare time. They were not required to identify themselves by name on the questionnaire. They were told truthfully that the HR department was interested in doing a "staff survey" and that their help was required. They were told that a report would be written to which they had access. In all the questionnaires took between 40 to 45 minutes to complete. The response rate in both organisations was over $80 \%$, which is considered to be a high level of response rate. Both received a written report on the findings which was disseminated to the participants. Very few questionnaires were incomplete or spoiled and no reports were received concerning the subjects inability to understand the instructions or that dissimulation occurred. Indeed many respondents had enjoyed the task and were extremely interested in the results.

\section{RESULTS}

Initially, each subscale's alpha coefficient of internal reliability was calculated to test the internal consistency of the inventories. Tables $7.2,7.3,7.4$, and 7.5 present descriptive 


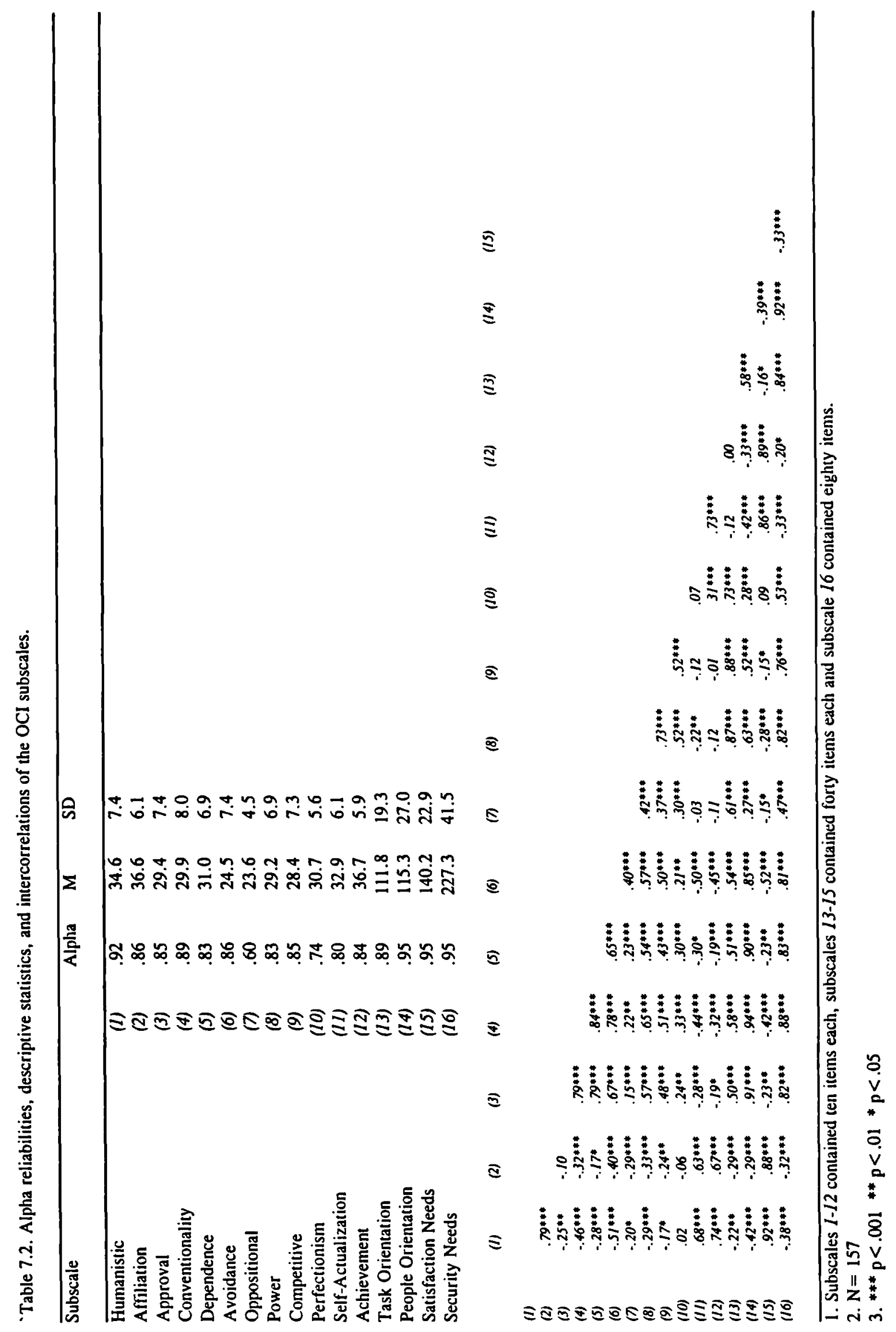




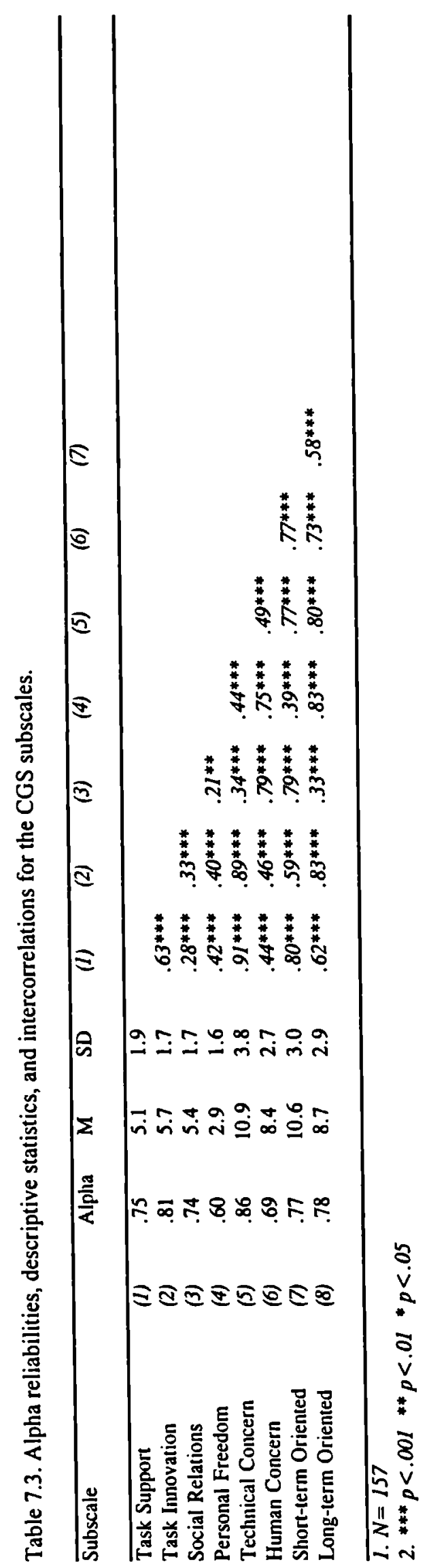




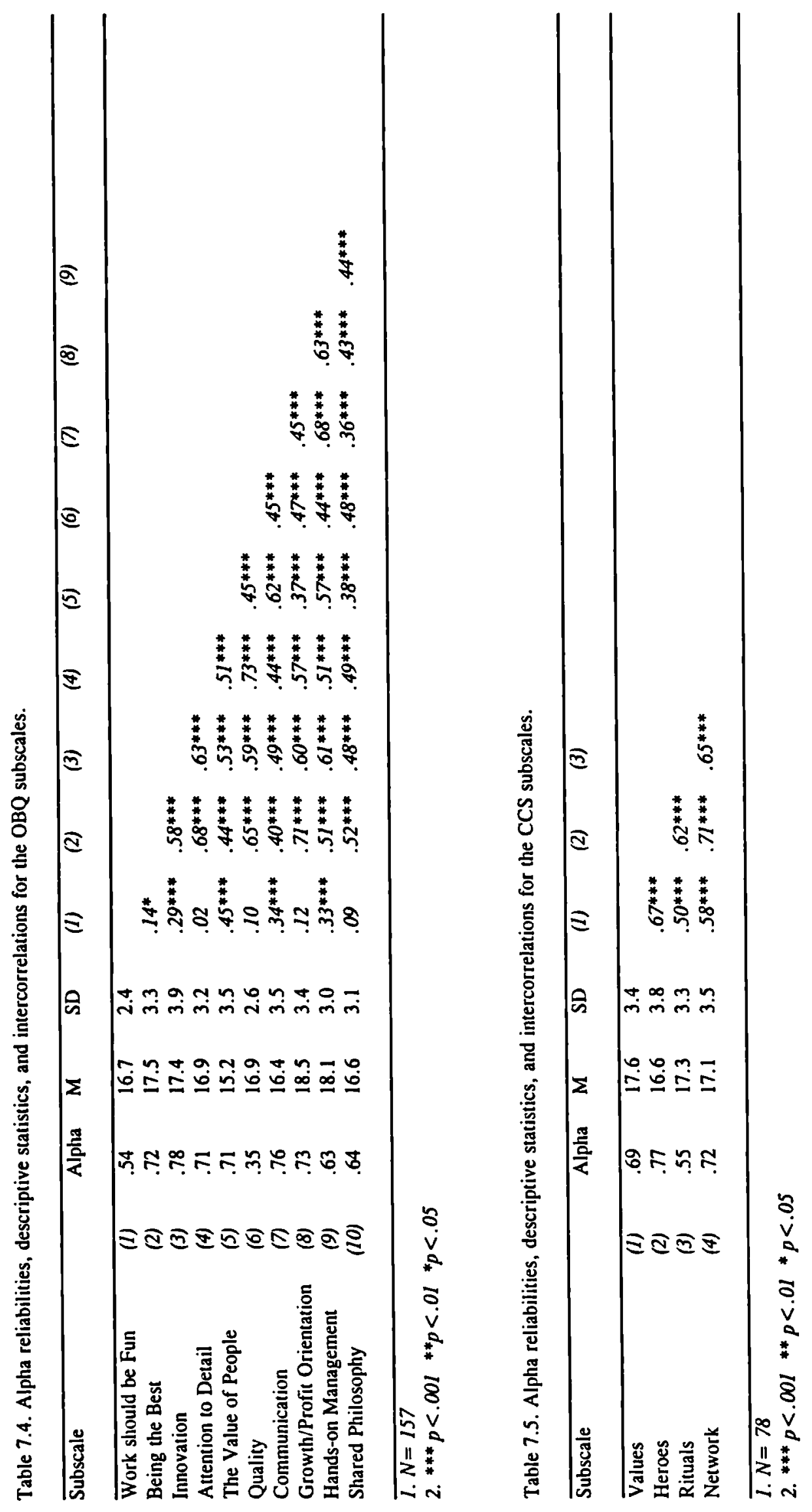


statistics and alpha reliabilities for all the questionnaires subscales, as well as intercorrelations among each questionnaire subscales. The OCI holds high to very high coefficients ranging from .60 to .95 , which means that the inventory shows high levels of internal consistency. Cooke and Rousseau (1988) have reported alpha reliabilities for the 12 basic subscales of the OCI ranging from .67 to .92 . Finally, it should be noticed that in the current study two of the secondary subscales, namely Satisfaction Needs and Security Needs, show very high coefficients of internal consistency (.95).

Moderate to high coefficients are generated by the CGS, the OBQ, and the CCS indicating that the respondents do not perceive the items comprising the subscales of these questionnaires as highly interrelated as the items contained in OCI's subscales. Consequently, the OCI seems to be the most reliable measure of organisational culture in comparison with the four questionnaires used in the current study mainly when the alphas of the four secondary OCI subscales are considered (.89 to .95$)$.

As far as the testing of hypothesis 1 is concerned, a correlational analysis was carried out to examine whether subscales that have a similar content were actually perceived by the respondents to be similar, providing information about the convergent validity of the inventories. As these correlations might have been affected by other variables, a set of partial correlations was performed aimed at partialling out in combination three variables, namely age, hierarchical level, and years of employment with the organisation. These variables have been shown in the relevant literature to be correlated with the way employees perceive the culture of their organisation. Table 7.6 shows the coefficients of partial correlations of the overlapping subscales with age, hierarchical level, and years of employment in the organisation partialled out. 
Table 7.6. Partial Correlations among the overlapping subscales of the $O C I$, the CGS, and the OBQ with age, hierarchical level, and years of employment in the organisation partialled out.

\begin{tabular}{llllr}
\hline Task Orientation & $(O C I)$ & / Technical Concern & $(C G S)$ & $-.40^{* * *}$ \\
People Orientation & $(O C I)$ & / Human Concern & $(C G S)$ & $-.48^{* * *}$ \\
Satisfaction Needs & $(O C I)$ & / Long Term & $(C G S)$ & $.52^{* * *}$ \\
Security Needs & $(O C I)$ & / Short Term & $(C G S)$ & $-.51^{* * *}$ \\
People Orientation & $(O C I)$ & / The Value of people & $(O B Q)$ & $-.53^{* * *}$ \\
Satisfaction Needs & $(O C I)$ & / Innovation & $(O B Q)$ & $.53^{* * *}$ \\
Human Concern & $(C G S)$ & / The Value of People & $(O B Q)$ & $.41^{* * *}$ \\
Long-term Orientation & $(C G S)$ & / Innovation & $(O B Q)$ & $.57^{* * *}$ \\
& & & & \\
\hline
\end{tabular}

$\overline{* * *} p<.001$

All the overlapping pairs of subscales concerning the behavioural norms' questionnaires show correlations, ranging from -.40 to .52 . More specifically, there are moderate correlations between the $\mathrm{OCI}$ and the CGS subscales measuring task orientation/technical concern $(-.40)$, people orientation/human concern $(-.48)$, satisfaction needs/long-term orientation (.52), and security needs/short-term orientation (-.51) at a .001 level of significance. The fact that the correlations are greater that .40 means that the two questionnaires measure quite similar theoretical constructs. However, three of these correlations are negative which means that the OCI and the CGS use different poles to measure the same cultural dimension. In other words what features as "high" on one scale features as "low" on the other.

Moreover, as far as the people orientation subscales are concerned, there is a negative correlation of -.53 between the $\mathrm{OCI}$ and the $\mathrm{OBQ}$ and a positive correlation of .41 between the CGS and the OBQ at a .001 level of significance. Finally, concerning the dimension of openness to change there are moderately high positive correlations between the OCI and the OBQ (.53), as well as between the CGS and the OBQ (.57) at a .001 level of significance.

Thirdly, a factor analysis was carried out in order to determine whether the various 
subscales are grouped in clusters showing possible dimensions of the concept of culture measured by the four questionnaires. This is perhaps the most widely used empirical multivariate method to discover the underlying structure of these different measures. The four subscales of the CCS were not included in the factor analysis since the questionnaire was completed only by the respondents of $\mathrm{OB}$. A principal components analysis with VARIMAX rotation was used to look at the structure of the 26 subscales. Five factors were extracted accounting for $69.9 \%$ of the total variance. The results are presented in Table 7.7.

Subscales of the questionnaires that contained items already included in the factor analysis were not used, the reason being that the information about culture provided by their items was already included. For example, the CGS subscale measuring Technical Concern was excluded from the factorial analysis as it contains items already included in the analysis through the subscales of Task Support and Task Innovation.

The first factor accounted for $41 \%$ of the variance and is identified by high loadings on seven subscales of the $\mathrm{OBQ}$. What distinguishes these subscales from the others seems to be their focus on the "values of excellent organisations," such as believing in being the best, promoting innovation, paying attention to detail and quality. The items seemed to involve the KAISEN philosophy imported from Japan which stresses continual improvement by a cautious but continuous change in a step-by-step manner. However, it should be noted that none of the people orientation subscales had their highest loading on this factor. The subscales of "avoidance" and "value of people" had high loadings on this factor, but had their highest loadings on factors three and five, respectively. This means that the factor refers to organisational growth which takes place by mainly concentrating on technical aspects of the job rather than the social relations developed in the workplace. Therefore, 


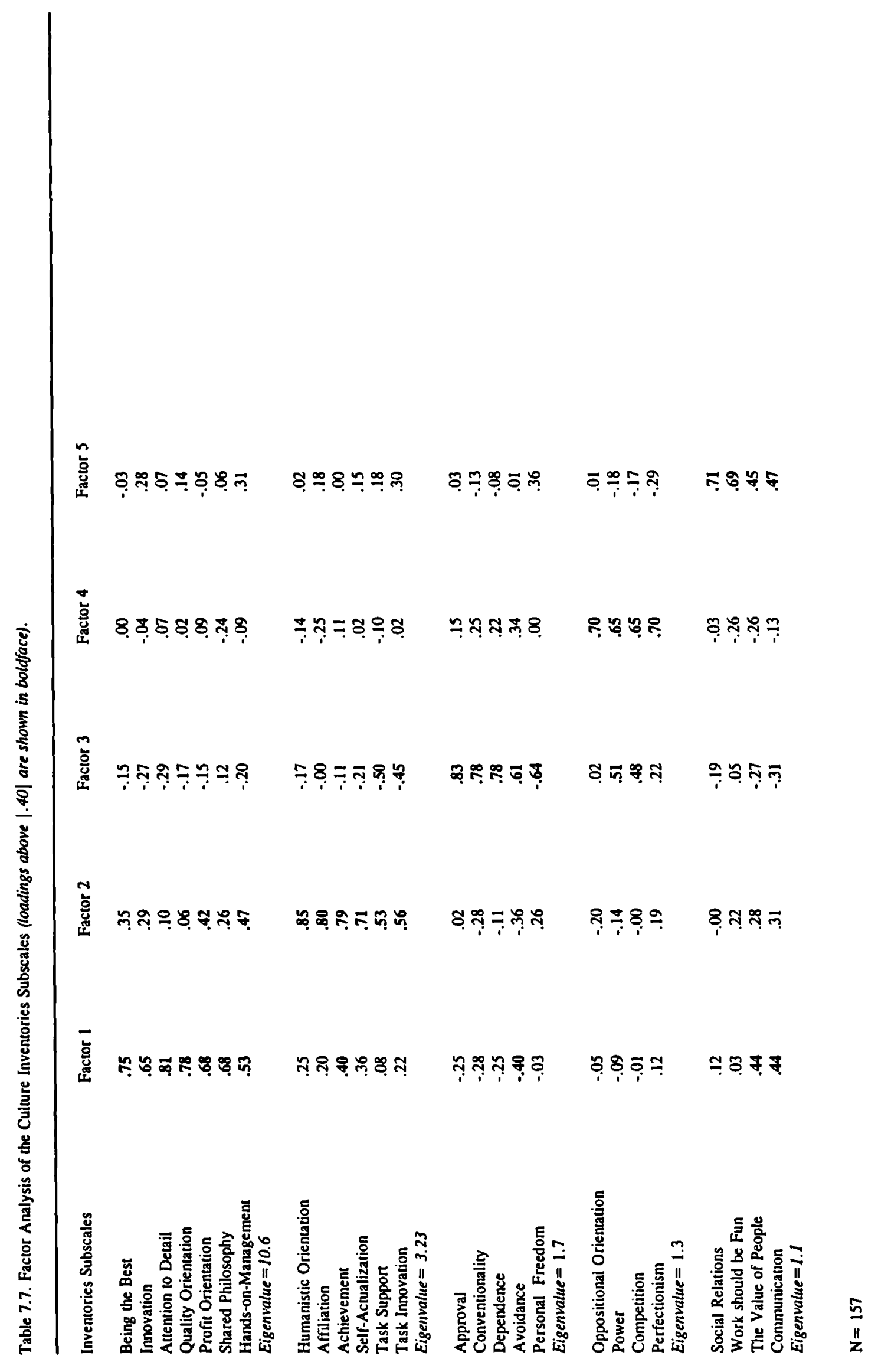


the factor could be labelled "task-oriented organisational growth".

The second factor contained four subscales of the $\mathrm{OCI}$ and two subscales from the CGS and accounted for $12.4 \%$ of the variance. The four subscales of the OCI loading on this factor form what the authors call secondary subscale of Satisfaction needs which concerns the tendency in an organisation to encourage members to innovate and take risks. The two subscales from CGS loading high on this factor measure task innovation and task support which is the degree of co-operation among the organisational members. Consequently, this factor tends to have subscales loading on it which stress openness to change, innovation, and achievement within a humanistic social environment where cooperation is highly valued and members are expected to be supportive and open to influence in their dealings with one another. While innovation and support belong to different conceptual schemes in the work of some theorists of organisational culture (Hofstede, 1986; Hofstede, Neuijen, Ohayv, \& Sanders, 1990) for Cooke and Lafferty (1989) they are both part of a "higher-order" factor called constructive styles or satisfaction needs.

The third factor was bipolar and accounted for $6.6 \%$ of the total variance. Four subscales of the OCI and one subscale of the CGS loaded on this factor. The subscales that loaded positively are conventionality, approval (do things to gain approval and acceptance), dependence (inability to take initiative), avoidance (shifting responsibilities to others and trying to avoid any possibility of being blamed for a mistake), while the subscale that loaded negatively is personal freedom. According to Cooke and Lafferty's model of organisational culture, the four subscales of the OCI loaded highly on this factor compose the "People Orientation" subscale. This term may be misleading and Cooke and Lafferty (1989) have noted that the four subscales - conventionality, approval, dependence, 
avoidance - may better be labelled "Passive/Defensive Styles." Moreover, as the factor mainly concerns conventionality, and formality, it appears to be similar with what Handy (1986) and Harrison (1972) call "Bureaucratic culture."

The fourth factor accounted for $5.4 \%$ of the variance and contained four subscales from the OCI. These four subscales seemed to focus on power, competition, confrontation, negativism toward the ideas of others, and desire to avoid all mistakes in order to be "perfect." In the Cooke and Lafferty model of culture, these four subscales comprise the dimension of task orientation. However, as oppositional orientation is one of the items which have the highest loading on this factor indicating that the core theme of this construct is negativism and as the subscales of power and competition have high loadings on factor 3 (people orientation), it could be argued that the factor might be measuring resistance to new ideas instead of task orientation. Moreover, this factor is negatively correlated with factor two $(r=-.24, p=.005)$, which concerns openness to change. Therefore, the members of the organisation maintain their status and influence (power-orientation) by being competitive and by opposing the ideas of others.

The final factor accounts for $4.5 \%$ of the common factor variance and contained one subscale from the CGS and three subscales of the OBQ. This factor appears to refer to socialising on the job, developing friendships with co-workers, participating in social activities with other members of the organisation and enjoying the job. Therefore, this factor focuses on positive social relations in the workplace.

Despite the fact that subscales from quite far disparate measures were concerned the results are interpretable with five factors emerging. These may serve the basis for the development of new measures for the comparison of already established measures. 


\section{DISCUSSION}

As far as the coefficients of internal reliability are concerned, the OCI demonstrated moderate to high levels of internal consistency ranging from .60 to .95 , while the CGS and the values questionnaires, namely $\mathrm{OBQ}$ and $\mathrm{CCS}$ have generated coefficients of internal consistency ranging from .35 to .86 . The partial correlations between the eight pairs of overlapping subscales with hierarchical position, years with the organisation and age partialled out, revealed modest correlations at a .001 level of significance. Regarding the factor analysis, six readily interpretable factors emerged accounting for $70.6 \%$ of the common factor variance.

Concerning the alpha reliabilities of the four questionnaire measures, the OCI secondary subscales, namely people orientation, task orientation, satisfaction needs, and security needs, show very high coefficients of internal consistency ranging from .89 to .95 . This finding indicates that when these subscales are used the questionnaire is the most internally reliable measure of organisational culture compared to the other three questionnaires used in the current study.

By taking into account the expressed concerns about an adequate testing of the convergent validity of questionnaire measures (Kilmann \& Thomas, 1977), a correlational analysis was carried out on the questionnaire subscales that intend to measure the same theoretical constructs instead of correlations between the total scores on each questionnaire. The main reason for doing this is the fact that the questionnaire constructors have developed different models of culture and therefore, they are tapping culture by measuring various cultural dimensions which might or might not be the same with the cultural dimensions 
measured by other questionnaire constructors. Consequently, low correlations between the total scores on each questionnaire can be the effect of the measurement of different dimensions of culture by the different questionnaires or the result of the inventories low convergent validity. In order to eliminate the effect that the measurement of different dimensions can have on the indicators of the convergent validity of the questionnaires the subscales that intend to measure the same construct were correlated.

The correlational analysis results suggested the acceptance of hypothesis 1. More specifically, all the overlapping pairs of subscales showed significant correlations $(-.40$ to .57) indicating that the subscales measure quite similar constructs. The results of the analysis testing the content similarity of the subscales which assess the same dimensions of organisational culture showed that these measures are significantly correlated. Consequently, the dimensions measured by various tests tend to be tapping the same phenomena when their contents overlap. The fact that the predicted correlations between the overlapping subscales of the inventories were supported by the data show to some extent the convergent validity of the questionnaire measures.

However, it should be noted that not all researchers would agree that all (or any) of these questionnaires are "tapping" organisational culture. Critics may argue that just because people answer roughly similar questions in similar ways about their perception of their organisation it does not follow that what they said necessarily expresses something to do with the culture of their organisation.

The higher-order factor analysis generated five readily interpretable factors accounting for $69.9 \%$ of the total variance. Although the number of respondents who participated in the current study is not ideal for conducting a factor analysis and the sample 
is quite restricted as far as the number of organisations and occupations included is concerned, the analysis can be justified by the fact that the generated factors can be interpreted by existing models of the organisational culture literature. Moreover, as the sample consists of full-time employed adults the results of the factor analysis have ecological validity.

As with all multivariate analysis the bigger the sample size the more stable and replicable the dimensions. This study examined subjects from only two organisations and it may be advisable to replicate this study in a larger, more representative sample. While it is likely that different sorts of respondents (i.e., more blue collar workers) from different sorts of organisations (i.e., very much smaller or more specialised) would perceive their organisation differently and provide a quite different questionnaire profile, the relationship between the subscales would remain much the same. That, however, is an empirical question and must await replication.

The first factor is identified by seven subscales of the OBQ which is based on the work done by Peters and Waterman (1982) to find the characteristics of those firms that are widely recognised for corporate excellence. The core theme of those six subscales appears to be a philosophy of continuous improvement and organisational development. As the factor does not contain any of the people-oriented subscales it is possible to suggest that it concerns mainly a technocratic approach of organisational development and thus can be called "task-oriented organisational growth."

The second factor consists mainly of the OCI secondary subscale labelled "Satisfaction Needs." According to the constructors of the inventory this subscale contains behavioural norms which encourage co-operation and reward supportive relations among 
the co-workers, while at the same time enhance innovation and change. Apart from "Satisfaction Needs" this factor contains the CGS subscales measuring task innovation and the degree of co-operation among the organisational members in order to get the job done. Therefore, it seems that this factor contains behavioural norms that are concerned with innovation and change within a co-operative environment and could be labelled "openness to change".

The third factor is composed of the OCI secondary subscale "People Orientation" and the CGS subscale concerning personal freedom in the workplace, which loaded on the factor negatively. As the "People Orientation" subscale measures conventionality and formalisation the dimension of culture tapped resembles, to a great extent, what Handy (1986) and Harrison (1972) called "Apollo culture" and "role culture", respectively. "Apollo culture" and "role culture" are typical bureaucracies. Therefore, it can be suggested that this factor concerns "the human factor in a bureaucratic culture."

The fourth factor consists of the OCI secondary subscale labelled "Task Orientation" which focuses on negativism towards the ideas of others, competition, perfectionism, and power. According to Cooke and Rousseau (1988), these norms of acceptable or unacceptable behaviour are mainly oriented on the job to be done (task orientation) and not on the people. However, two of the factor items, namely power and competition have high loadings on the third factor which contains the OCI secondary subscale "People Orientation." Accordingly, it can be argued that the factor concerns mainly "resistance to new ideas" rather than "Task Orientation." Negativism towards the ideas of others and rough competition is used by the organisational members to maintain or gain power.

The final factor yielded by the analysis is a combination of subscales from the CGS 
and the OBQ. The factor appears to stress on the human relations in the organisation, the development of friendships among the co-workers, and the general issue of socialising in the work setting. Moreover, it refers to the idea that work should be fun and that organisational members should enjoy what they are doing. The label given to this factor is "positive social relations in the workplace."

The aim of the factor analysis was to examine whether the various dimensions of culture suggested by the models on which the development of each scale was based, could be integrated into a framework of the organisational culture dimensions. The results supported a five-dimensional model of organisational culture: task-oriented organisational growth, openness to change, resistance to change, the human factor in a bureaucratic culture, and positive social relations in the workplace.

The current study could be used to lead one to recommend some measures over others. The OCI had the best internal reliability and its subscales loaded on far of the five dimensions, while the CCS had poorest alphas.

Finally, two of the factors that emerged from the factor analysis, that is, "openness to change" and "resistance to change" contain behavioural norms that refer to whether organisational members are expected to take risks and innovate or resist new ideas. The subscales comprising these factors will be used to test the effect of organisational norms, which either facilitate or inhibit innovation, on individuals' motivation to be creative at work. 


\section{CHAPTER EIGHT}

THE EXTENSION OF AMABILE'S CREATIVITY

THEORY WITHIN ORGANISATIONAL BEHAVIOUR:

ORGANISATIONAL CULTURE AND THE

MOTIVATION TO BE CREATIVE 


\section{INTRODUCTION}

This study concerns the further elaboration of the proposed extension of Amabile's creativity theory within the framework of organisational behaviour. The extension of Amabile's theory is an attempt to incorporate social factors that promote creative behaviour. Amabile, Goldfarb, and Brackfield (1990) acknowledged that the social psychology of creativity has focused on the detrimental effect of social factors on creativity, while neglecting any positive influence of these factors on creative behaviour.

The theory's extension tested in chapter six of this thesis refers to the identification of pro-creativity social norms as a variable that promotes motivation following failure in a creativity task. Pro-creativity social norms affect after-failure motivation via their impact on the perception of the attributions for failure. The presence of pro-creativity social norms leads to the attribution of failure to unstable and changeable causes, which, in turn, prevents the impairment of subsequent motivation. Groups that have pro-creativity social norms encourage their members to explore new ways of doing things which increases the chance of experiencing failure. Therefore, since failure is encountered in such groups, members can compare their own failures to the failures of their colleagues and perceive the causes of these failures as unstable and changeable. On the other hand, anti-creativity social norms are related to the attribution of failure to stable and unchangeable causes, which decreases the levels of after-failure motivation. Since in anti-creativity groups avoiding failure is more important than pursuing change and development, members are not able to compare their failure to the failures of others which leads to the perception of failure as caused by stable and unchangeable factors. 
Organisational social norms have been extensively studied as an element of the concept of organisational culture. Even though the concept of organisational culture has a number of manifestations/elements, i.e., social norms, values, basic assumptions, artifacts, researchers usually choose to study one of these elements depending on which they believe to be more accessible or informative of the construct of culture (see chapter 3). Cooke and Rousseau (1988) have studied the social norms that dictate what an organisational member needs to do to fit in the group on the grounds that social norms are more accessible compared to other manifestations of culture. Schein (1985) is interested in the basic assumptions that are unconsciously held and lead the behaviour of group members because he argues that basic assumptions are the most informative manifestations of culture. It has been suggested in the organisational culture literature that a core theme exists between the various elements of culture. For example, when there is an implicit belief (basic assumption) that innovation is important for the development of the organisation, then innovation is probably valued (value), and members are expected to be innovative (social norm).

Since organisational social norms are recognisable and measurable manifestations of the concept of organisational culture and a common theme exists between social norms and the other elements of culture, the literature concerning the effect of organisational culture on innovation can be illustrative for the purpose of identifying organisational social norms that facilitate creativity at work.

At this point the relationship between the concepts of creativity and organisational innovation needs to be clarified. Innovation can be an absolute or a relative novelty; that is, it might concern bringing into existence something totally new (absolute novelty), or implementing a well-known idea which is new to the relevant unit of adoption (relative 
novelty). Innovation as an absolute novelty, rather than adaptation, involves creativity at its ideation component (West \& Farr, 1990). Therefore, as far as the ideation component of innovation is concerned, there is a conceptual overlap between innovation and creativity .

Concerning the effect of organisational culture on innovation, Kanter (1983) proposed that innovation can not flourish unless the organisation values change, and emphasised the importance of internal co-operation and achievement motivation for people and organisations to be innovative. Cooke and Rousseau (1988) suggested that innovation is enhanced by a culture that has a humanistic orientation and encourages self-actualization and achievement. West and Anderson (1992) found that a culture that emphasises creativity and growth (developmental culture), as well as a culture that is people-oriented (group culture), are perceived by raters (employees of an organisation and postgraduate students) to be more innovative environments compared to a culture that emphasises achievement and task accomplishment (rational culture) or the importance of formality and stability (hierarchical culture). Moreover, Amabile (1988) suggested that an organisational climate which is characterised by the shared perceptions of organisational members that: (a) creativity is important, and (b) internal co-operation facilitates the exploration of new ideas, is conducive to an individual's motivation to be creative.

In addition, it has been suggested by the relevant literature that an organisational culture which is characterised by centralisation of power and internal competition has a detrimental effect on organisational innovation. Cooke \& Rousseau (1988) proposed that centralisation of power and internal competition are "defensive strategies" which inhibit innovative behaviour. Damanpour (1987), in a meta-analysis of the relationship between innovation and its determinants, found that centralisation of power is negatively related to 
innovation. Finally, Kanter (1983) has emphasised the importance of a decentralised organisation for innovation to flourish.

Xenikou and Furnham (in press), in an attempt to identify the dimensions of the concept of organisational culture, found that organisational norms concerning creativity, internal co-operation, and achievement cluster together comprising the dimension of culture that the authors labelled as "openness to change." Moreover, the organisational norms regarding centralisation of power and internal competition are incorporated in another dimension of organisational culture labelled as "resistance to change."

Therefore, it is possible to suggest that pro-creativity social norms, as well as norms promoting internal co-operation and achievement, are facilitators of innovation, while centralisation of power and internal competition are reported as inhibitors of innovation. Since suggesting that innovation is facilitated by pro-creativity social norms might seem tautological, it has to be clarified that the facilitation of innovation occurs by the co-existence of the organisational norms encouraging creativity, internal co-operation, and achievement and not by the sole presence of pro-creativity norms. Moreover, West and Anderson (1992) reported that an organisational culture which is achievement-oriented, is not perceived by raters as innovative as a culture that emphasises creativity or internal co-operation. However, in this thesis it is suggested that the negative effect of achievement orientation on innovation is eliminated or even reversed into a positive effect, when the organisation values achievement, as well as social support and creativity.

The term openness to change will be utilised for referring to the co-existence of procreativity norms, norms of co-operation, and norms of achievement; the term resistance to change will be used for referring to the co-existence of norms promoting centralisation of 
power and competitive behaviour. Since it was shown by Xenikou \& Furnham (in press) that openness and resistance to change form separate dimensions of the concept of culture and not the opposites of the same dimension, they are considered as two different factors.

The extension of Amabile's creativity theory tested in chapter six is further elaborated within organisational behaviour. The elaboration concerns the examination of the effect of organisational culture on employees motivation to be creative following failure in a creativity task. An organisational culture that is characterised by openness to change leads to unstable attributions for failure, which in turn lead to the maintenance of intrinsic motivation for similar tasks. In addition, since organisational culture researchers have suggested that innovation is inhibited by the centralisation of power and internal competition, it is suggested that resistance to change leads to stable attributions for failure. The above hypothesised relations can theoretically be supported by the possible interference of the process of social comparison. Organisational groups which facilitate innovation by having norms of openness to change might experience a bigger number of failures compared to groups which inhibit innovation by having norms of resistance to change, simply because the latter are more interested in avoiding failure than succeeding. Therefore, when failure in a creativity task is encountered, members of the first groups have access to a sufficient number of comparison targets (other members who failed in the past) leading to unstable attributions for failure, while members of the second groups have very limited access to such targets, which leads to stable attributions for failure.

Even though the extension of the creativity model presented in chapter 6 concentrated on the positive effect of pro-creativity norms on after-failure motivation via causal stability, the elaboration of the extension of the model attempted in this study includes another factor 
which is, according to Weiner's attributional theory, a determinant of the levels of motivation, namely, expectancy of success. Thus, social norms are hypothesised to have an indirect effect on after-failure motivation via their impact on causal stability, as well as expectancy of success.

By including the effect of social norms on future expectancy of success an attempt is made to consider that groups which have norms of openness to change do not only experience more failures compared to groups with norms of resistance to change, but also more successes. Norms of openness to change are related to more successes compared to norms of resistance to change, simply because the latter focuses on maintaining the "status quo" rather than accomplishing success. Since groups characterised by openness to change experience success at least more frequently compared to groups with norms of resistance, they develop a belief of collective efficacy (Lawson \& Ventriss, 1992). To Lawson and Ventriss (1992), "organisational cultures stressing innovation and change enhance not only perceived selfefficacy but also perceived collective efficacy, that is, an organisational member's assessment of the capability of the organisation to execute specific performances" (p. 216). Therefore, an organisational culture that is characterised by openness to change helps members to develop beliefs about the collective efficacy of the organisation which in turn lead to the formulation of high expectancy of success after failing in a creativity task. On the other hand, an organisational culture characterised by resistance to change has lower levels of perceived collective efficacy, which is associated with low expectancy of future success following a failure event.

The aim of the current study is to elaborate the proposed extension of the creativity model within organisational behaviour. In order to accomplish the above aim the following 
hypotheses were tested:

Following the rejection of one's creative idea at work:

Hypothesis1 Given that the initial motivation to engage in the creativity task is high, unstable attributions for failure lead to high levels of subsequent motivation, while stable attributions for failure lead to low levels of subsequent motivation.

Hypothesis 2 High expectancy of future success is associated with high levels of subsequent motivation, whereas low expectancy of future success is associated with low levels of subsequent motivation.

Hypothesis 3 Given that the initial motivation to engage in the creativity task is high, organisational norms of openness to change lead to unstable attributions for failure.

Hypothesis $4 \quad$ Organisational norms of openness to change lead to high expectancy of future success.

Hypothesis 5 Given that the initial motivation to engage in the creativity task is high, organisational norms of resistance to change lead to stable attributions for failure.

Hypothesis $6 \quad$ Organisational norms of resistance to change lead to low expectancy of future success. 
Since the above hypotheses were tested by using a path analysis (see results section) figure 8.1 shows which paths were testing each of the six hypotheses.

The testing of hypotheses 5 and 6 does not address the issue of the extension of Amabile's creativity theory in order to incorporate social facilitators of creativity since it concerns the detrimental effect of organisational norms of resistance to change on motivation to be creative. However, by testing whether resistance to change has a negative effect on after? failure motivation via causal stability and expectancy of future success additional evidence may emerge to support the conducive effect of openness to change on motivation to be creative. Therefore, if resistance to change is proven to be associated with low levels of motivation following failure, this finding may serve as additional evidence for the positive effect of openness to change on shift in intrinsic motivation.

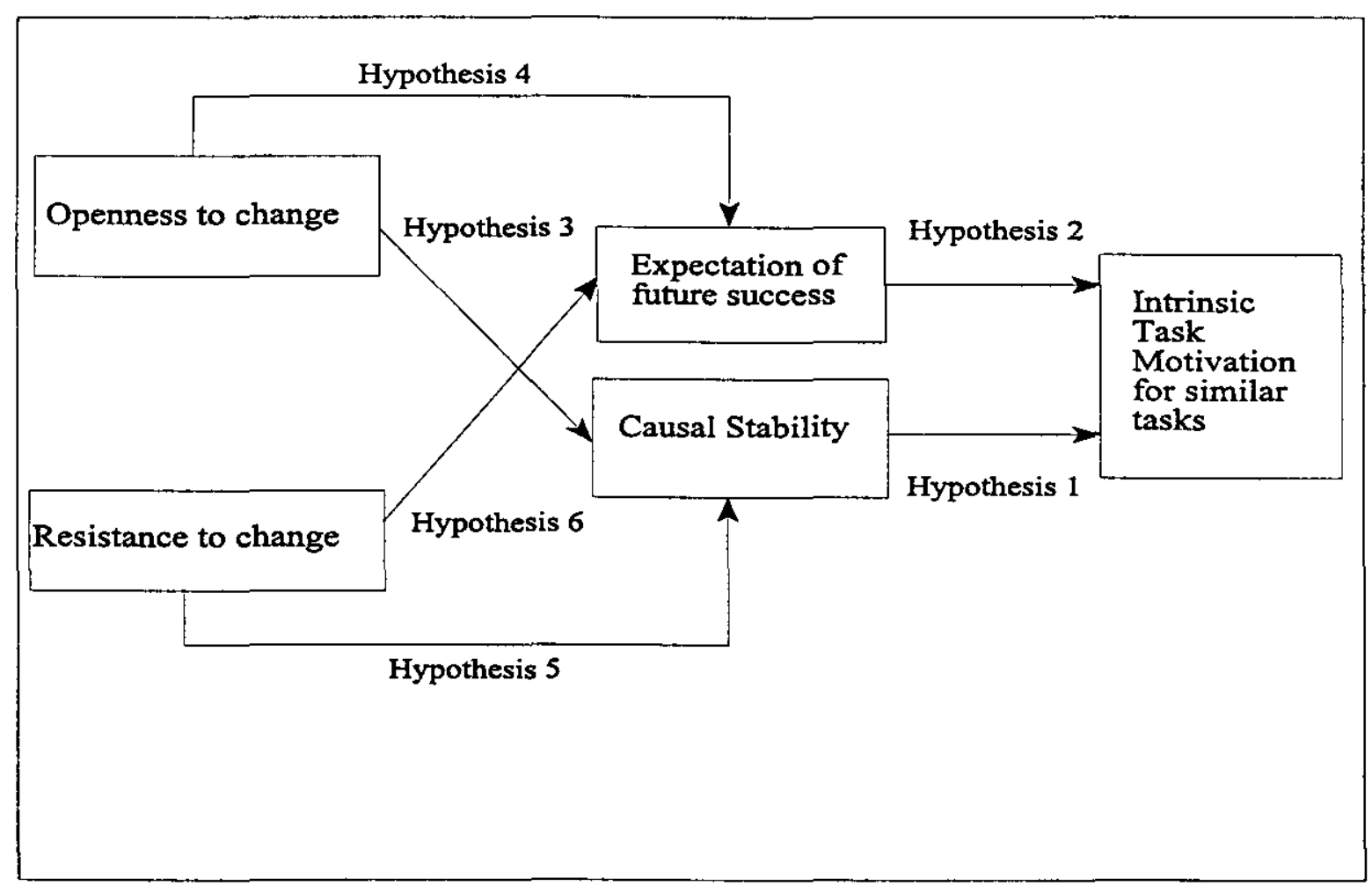

Figure 8.1. Graph showing the correspondence of hypotheses and paths. 


\section{METHOD}

Respondents. A total of 50 employees of two financial organisations and a consultancy firm took part in this study. There were $18(37.5 \%)$ females and $30(62.5 \%)$ males in the group. Concerning their age, $17(34.7 \%)$ were 20 to 29 years old, $23(46.9 \%)$ were 30 to 39 years old, $5(10.2 \%)$ were 40 to 49 years old, and $4(8.2 \%)$ were 50 to 59 years old. As regards their hierarchical level, 14 (33.3\%) occupied non-management positions, $12(28.6 \%)$ occupied line management positions (supervising non-management personnel), and $16(38.1 \%)$ were middle managers (managing managers) and senior managers. Finally, 14 employees $(29.2 \%)$ were less than 1 year with the organisation, $15(31.2 \%)$ were from 1 to 6 years, and $19(39.6 \%)$ were members of the organisation for over 6 years.

Questionnaires. Respondents were required to provide the main cause of a provided failure scenario and to rate this cause on the Revised Causal Dimension Scale's ( (CDSII; McAuley, Duncan, \& Russell, 1992) subscale of causal stability. In addition, shift in intrinsic motivation following the failure event, expectancy of future success, organisational norms encouraging openness to change, and organisational norms promoting resistance to change were measured. Finally, the respondents were asked to rate their interest in finding new ways to improve the way their job is being done.

Assessment of causal stability in the case of failure. In order to measure the subjective stability of the major cause to which the rejection of the creative idea was attributed, the following scenario was used: 
The management of the company is concerned about the improvement of customer service. In order to collect as many ideas about customer service as possible, the management has decided to use "suggestion boxes." Any individual employee who is interested and feels challenged by the task of finding new ways to serve customers, has the opportunity to write an extensive proposal of her/his ideas and put it in the suggestion box. Each of the proposed ideas is discussed thoroughly by the management and is either accepted or rejected for application. The proposals are anonymous, so that the hierarchical position, occupation, or status of the employees won't interfere in the process of management decision making. However, each proposal has a code on it and these codes are used for the announcement of whether a proposal was accepted or rejected. Employees whose proposals are accepted for application are expected to contact the management.

The scenario emphasised that the task is undertaken by individuals who are interested in it. In addition, the scenario suggested that the process of selecting proposals was anonymous, so that position in the organisation, occupation, or status of the employees would not interfere.

The respondents were asked to imagine that they were very interested by the task of finding new ways to serve the customers and that they proposed a new idea which, however, was rejected. Then, they were asked to provide the major cause for this failure and rate its stability. The CDSII's (McAuley, Duncan, \& Russell, 1992) subscale of causal stability was used for the measurement of the stability of the provided cause. The CDSII's subscale of causal stability contains three items rated on a 7-point scale. McAuley, Duncan, and Russell (1992) reported a coefficient of alpha reliability of .67 for the subscale of causal stability, and demonstrated the validity of the questionnaire by a confirmatory factor analysis. The coefficient of alpha reliability obtained in the current study was .48 , which is not acceptable (Nunnally, 1978). 
Shift in intrinsic motivation. The shift in intrinsic motivation following the rejection of one's idea was measured by the change of how interesting and challenging the respondent perceived the task to be. In other words, after the rejection of the idea would it become more or less interesting and challenging (two items) to look for new ways to improve the company's operation.

Amabile (1985) assesses intrinsic motivation by measuring the subjects interest in the task, how challenging they perceive the task to be, and finally, the degree of satisfaction that they get out of it. In the current study the degree of satisfaction is not incorporated as an index of intrinsic motivation, since failure itself leads to a negative affect (Weiner, 1986). The coefficient of alpha reliability obtained in this study was .81 which is acceptable.

Expectation of future success. Expectation of future success following a failure event was measured by a single item rated on a 7-point scale. The item was: "Would you expect that next time you propose a new idea it will be accepted for application ?" The anchors of the 7point scale were: Will be accepted and will not be accepted. High scores indicate high expectation of future success and low scores indicate low expectation.

Openness to change. In order to measure organisational norms regarding openness to change the organisational culture's themes of individual creativity at work, internal co-operation /humanistic orientation, and achievement orientation had to be measured (Xenikou \& Furnham, in press).

The Culture Gap Survey's (CGS; Kilman \& Saxton, 1983) subscale labelled "task innovation" was utilised for measuring creativity at work. The alpha reliability of the subscale 
was reported to be .89 (Saxton, 1987), and .81 (Xenikou \& Furnham, in press). The subscale of task innovation contains 7 items, rated on a dichotomous scale.

Internal co-operation/humanistic orientation and achievement orientation were measured by the Organisational Culture Inventory's (OCI; Cooke \& Lafferty, 1989) subscales labelled humanistic/helpful, and achievement. Each subscale is composed of 10 items, rated on a 5-point Likert scale. Cooke and Rousseau (1988) reported alpha coefficients of .90 and .85 for the subscales of humanistic/helpful and achievement, respectively. Xenikou and Furnham (in press) found that the coefficients of the alpha reliability of the humanistic/helpful and the achievement subscales were .92 and .84 , respectively. The alpha coefficient of the openness to change scale obtained in this study was .87 .

Resistance to change. Organisational norms promoting resistance to change concern the centralisation of power and internal competition. Damanpour (1991) showed that the centralisation of power is negatively related to innovation and Xenikou and Furnham (in press) found that both the organisational culture themes of centralisation of power and competition are incorporated in a general factor which they called "resistance to change." Therefore, organisational norms promoting resistance to change were measured by the OCI's subscales labelled power and competition. The subscales are composed of 10 items, rated on a 5-point Likert scale. The reported alpha coefficients for the power subscale were .80 (Cooke \& Rousseau, 1988) and .83 (Xenikou \& Furnham, in press). The reported alpha reliability for the subscale of competition were .82 (Cooke \& Rousseau, 1988) and .85 (Xenikou \& Furnham, in press). The coefficient of alpha reliability obtained in this study was .94 , which is very high. 
Personal interest in being innovative. Respondents were asked to rate on a 7-point scale the degree to which they are interested in finding new ways to improve the way their job is being done (low scores indicating low interest and high scores indicating high interest). Since it was demonstrated in study 1 of this thesis that causal stability is a predictor of after-failure motivation provided that the initial motivation to engage in the task is high, the respondents that were not interested in being innovative should be excluded from any analysis involving causal stability. Only one respondent reported that his interest was below 5 and was thus excluded from the analysis.

Procedure. The respondents were asked to complete the questionnaires at work or during their spare time. In all the questionnaire took between 30 to 45 minutes to complete. The respondents did not have to identify themselves by name on the questionnaire, but they could if they wanted to receive personal feedback. A report was also written concerning the overall results of the study to which all the respondents had access.

\section{RESULTS}

A path analysis was carried out in order to test the hypotheses of this study. The Pearson correlations among the variables included in the model are presented in the following table. 
Table 8.1. Pearson correlations among shift in intrinsic motivation (SIM), expectancy of success (ES), causal stability (CS), openness to change (OC), and resistance to change (RC).

\begin{tabular}{lcrllll}
\hline & M & SD & SIM & ES & CS & OC \\
SIM & 8.73 & 3.46 & & & & \\
ES & 3.81 & 1.42 & $.48^{* * *}$ & & & \\
CS & 10.81 & 3.55 & -.11 & .08 & & \\
OC & 81.45 & 11.39 & -.07 & $.37^{*}$ & .01 & \\
RC & 57.75 & 16.85 & -.00 & $-.32^{*}$ & -.00 & -.24 \\
\hline
\end{tabular}

$* \mathrm{P}<.05 * * * \mathrm{P}<.001$

Firstly, a multiple regression was conducted with shift in motivation following the rejection of one's idea as the dependent variable. The independent variables were causal stability, expectancy of future success, and norms promoting openness to change or resistance to change. This regression tests whether any of the four predictors have a direct effect on shift in intrinsic motivation. This regression analysis tests hypothesis 1 and 2; that is the direct effect of causal stability and expectancy of future success on levels of subsequent motivation. A squared multiple correlation coefficient of $\mathrm{R}^{2}=.43, \mathrm{~F}(4,35)=6.62, \mathrm{p}<.001$ was found. The results are presented in table 8.2 .

The results show that when an employee's idea is not accepted (a failure situation) his/her expectation for future success is a significant predictor of the shift in motivation to engage in similar tasks. More specifically, high expectation for future success is associated with high levels of motivation to carry out a similar task, while low expectation for future success is related to low levels of motivation. Therefore, expectancy of future success was shown to have a direct effect on shift in intrinsic motivation supporting hypothesis 2 . 
Table 8.2. Multiple regression analysis. Dependent variable: Shift in intrinsic motivation.

\begin{tabular}{lrrl}
\hline Variable & Beta & $\mathrm{t}$ & Sig t \\
\hline Causal Stability & -.21 & -1.69 & .098 \\
Expectation of success & .73 & 5.05 & .000 \\
Openness to change & -.16 & -1.21 & .232 \\
Resistance to change & .26 & 1.86 & .070 \\
$\mathrm{R}^{2}=.43, \mathrm{~F}(4,35)=6.62$, & & & \\
$\mathrm{p}=.0004$ & & & \\
& & & \\
$\mathrm{~N}=40$ & &
\end{tabular}

In the second multiple regression, causal stability was regressed on organisational norms encouraging either openness or resistance to change testing hypotheses 3 and 5; that is the direct effect of openness and resistance to change on causal stability. None of the two variables emerged as a significant predictor of causal stability of failure. The results are presented in table 8.3 .

Table 8.3. Multiple regression analysis. Dependent variable: Causal stability.

\begin{tabular}{llll}
\hline Variable & Beta & $\mathrm{t}$ & Sig t \\
\hline Openness to change & .06 & .359 & .721 \\
Resistance to change & .03 & .209 & .835 \\
$\mathrm{R}^{2}=.00, \mathrm{~F}(2,37)=.07$, & & & \\
$\mathrm{p}=.931$ & & & \\
& & & \\
$\mathrm{~N}=40$
\end{tabular}

Finally, a third multiple regression was carried out to examine the effect of organisational norms which either facilitate (openness to change) or inhibit creativity (resistance to change) on expectancy of future success (hypotheses 4 and 6). This regression tests whether openness to change and resistance to change have an effect on expectancy of 
future success. If openness to change and/or resistance to change emerge as significant predictors of expectancy of success, then it is possible to suggest that they indirectly influence shift in intrinsic motivation through their impact on expectancy of success since firstly, expectancy of success is a significant predictor of shift in motivation and secondly, openness and resistance to change do not have a direct effect on motivation. An $R^{2}=.20, F(2,38)=$ $4.89, p=.012$ was found. The results are presented in table 8.4.

Table 8.4. Multiple regression analysis. Dependent variable: Expectation of success.

\begin{tabular}{lcrl}
\hline Variable & Beta & $\mathrm{t}$ & Sig t \\
\hline Openness to change & .27 & 1.85 & .071 \\
Resistance to change & -.29 & -2.00 & .052 \\
$\mathrm{R}^{2}=20, \mathrm{~F}(2,38)=4.89$, & & \\
$\mathrm{p}=.012$ & & & \\
& & & \\
\hline $\mathrm{N}=41$
\end{tabular}

The joint effect of norms concerning openness to change and resistance to change on expectation for future success following failure was found to be significant. This finding indicates that the presence of organisational norms which encourage innovation and the absence of norms which inhibit inovation are related to high expectation for future success when failure has been encountered.

Since the scale of causal stability did not reach the acceptable levels of alpha reliability (Nunnally, 1978) the above findings can be questioned on the basis of measurement error related with this particular scale. Therefore, a new path analysis was conducted after excluding the scale of causal stability. Hypotheses 1,3 , and 5 concerning the causal relations between causal stability and the other psychological constructs could not be tested in this 
study since a reliable measure of causal stability was not available.

A multiple regression was carried out to test the effect of expectancy of future success and social norms (openness to change, resistance to change) on shift in intrinsic motivation following failure testing for any direct effect of the three variables on shift in intrinsic motivation. This analysis tested hypothesis 2 . An $\mathrm{R}^{2}=.38, \mathrm{~F}(3,38)=7.87, \mathrm{p}=.0003$ was calculated.

Table 8.5. Multiple regression analysis. Dependent variable: Shift in intrinsic motivation.

\begin{tabular}{lccl}
\hline Variable & Beta & $\mathrm{t}$ & Sig t \\
\hline Expectancy of success & .69 & 4.85 & .000 \\
Openness to change & -.21 & -1.54 & .131 \\
Resistance to change & .19 & 1.41 & .164 \\
$\mathrm{R}^{2}=.38, \mathrm{~F}(3,38)=7.87$, & & & \\
$\mathrm{p}=.0003$ & & &
\end{tabular}

$\mathrm{N}=42$

Following the rejection of one's idea, higher expectation for subsequent success leads to higher levels of intrinsic motivation to engage in similar tasks, while lower expectation of subsequent success is related to lower levels of intrinsic motivation. Organisational norms did not emerge as a significant predictor of shift in intrinsic motivation. Therefore, only expectancy of future success has a direct effect on shift in intrinsic motivation.

Finally, a hierarchical multiple regression was carried out in order to examine the effect of organisational norms which either encourage openness or resistance to change on expectation for subsequent success testing hypotheses 4 and 6 . The hierarchical multiple regression technique allows us to make a choice based on theoretical grounds concerning the distribution of the dependent variable's variance which is explained by both the predictors. Even though openness to change and resistance to change emerged as separate factors of the 
construct of organisational culture (Xenikou \& Furnham, in press), they were negatively correlated $(r=-.24, p=.005)$. When the predictors are correlated, it is likely that the portion of the criterion's variance commonly explained by the predictors is bigger. In this case a decision can be made on theoretical grounds for the distribution of the commonly explained variance. Regarding the specific hierarchical regression analysis, it was decided the common variance to be given to the organisational norms that promote openness to change as the presence of facilitators of innovation may be more important than the absence of inhibitors. The results are presented in table 8.6.

Table 8.6. Hierarchical multiple regression. Dependent variable: Expectation for success.

\begin{tabular}{llll}
\hline Variable & Beta & $\mathrm{t}$ & Sig t \\
\hline $\begin{array}{l}\text { Block } 1 \\
\mathrm{R}^{2}=.11, \mathrm{~F}(1,40)=5.31, \\
\mathrm{P}=.026\end{array}$ & & \\
Openness to change & & \\
Block 2 & .34 & .026 \\
$\mathrm{R}^{2}=.20, \mathrm{~F}(2,39)=5.14$, & & \\
$\mathrm{p}=.010$ & & \\
Resistance to change & & \\
$\Delta \mathrm{R}^{2}=.09, \mathrm{~F}(1,39)=4.83$, & & .040 \\
$\mathrm{p}<.05$ & -.31 & -2.12 & \\
\hline $\mathrm{N}=42$ & & \\
\end{tabular}

Openness to change emerged as a significant predictor of expectation for subsequent success following failure with a positive beta weight of .34 , while resistance to change has a negative beta weight of -.31 . These results indicate that the presence of organisational norms that promote openness to change leads to high expectation for subsequent success following failure, whereas the presence of norms that encourage resistance to change is associated with 
low expectation for future success. Therefore, on the one hand an organisational culture that encourages individual creativity, internal co-operation, and achievement (openness to change) is related to members high expectation of success when failure has been encountered, and on the other a culture that is power-oriented and competitive (resistance to change) leads its members to expect that failure will be followed by failure and not by success.

Since expectancy of future success was shown to be a significant predictor of shift in intrinsic motivation and the factors of openness to change and resistance to change emerged as significant predictors of expectancy of future success, it is possible to suggest that there is an indirect effect of openness and resistance to change on shift in motivation via expectancy of success. Also it has to be noted that openness and resistance to change did not have a direct effect on subsequent levels of motivation and therefore, there is only an indirect effect of these variables on motivation through expectancy of future success.

The model of the causal relationships between shift in motivation following the rejection of one's idea, expectancy of future success, openness to change and resistance to change is presented in figure 8.2 .

Figure 8.2 shows that expectancy of future success is a good predictor of shift in intrinsic motivation with a positive beta weight of .69 which indicates that high expectancy of future success leads to high levels of after-failure motivation, while low expectancy of success leads to low levels of motivation. In addition, openness to change has a positive indirect effect on shift in intrinsic motivation via expectancy of success, whereas resistance to change has a negative indirect effect on after-failure intrinsic motivation through expectancy of subsequent success. 


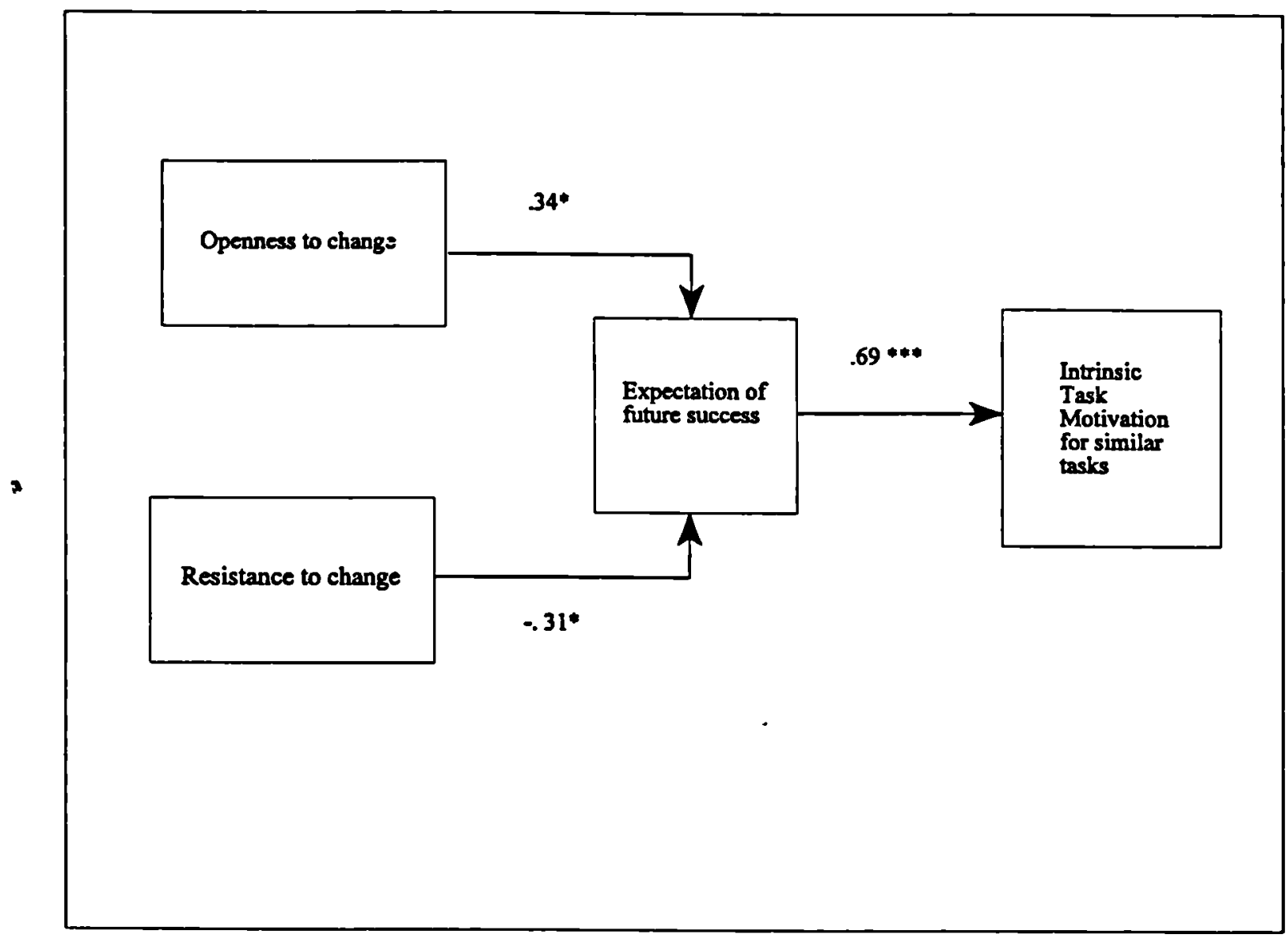

Figure 8.2. The indirect effect of organisational norms on shift in intrinsic motivation via expectation of future success.

$* p<.05 * * * p<.001$

\section{DISCUSSION}

The findings of this study show that various facets of organisational culture have an effect on motivation to be creative at work through the formulation of expectancies of future success, supporting hypotheses 4 and 6 . An organisational culture which is characterised by openness to change has a positive indirect effect on after-failure motivation to be creative via its impact on expectancy of success. That is, the presence of organisational norms that promote openness to change leads to higher expectancy of subsequent success following the rejection of one's creative idea, while the absence of such norms leads to lower expectancy 
of subsequent success. On the other hand, an organisational culture that is characterised by resistance to change has a negative indirect effect on after-failure motivation to be creative through its impact on expectancy of success. In other words, the presence of organisational norms promoting resistance to change is related with low expectancy of future success, whereas the absence of resistance to change is associated with high expectations for success.

The hypotheses $(3 \& 5)$ concerning the effect of openness to change and resistance to change via causal stability could not be tested because the measure of causal stability was shown to be unreliable. Even though the CDSII's (McAuley, Duncan, \& Russell, 1992) subscale of causal stability was used in two other studies of this thesis showing acceptable levels of alpha reliability (study $1:$ alpha $=.76$ and study $3:$ alpha $=.79$ ), the alpha reliability in this study was .48 , which is below the acceptable level (Nunnally, 1978). One difference between studies one and three on the one hand, and the current study on the other is that this study had a sample of working people, while the other two studies employed a sample of undergraduate or Ph.D. students. It is, therefore, possible to suggest that working people are more sensitive to differences between a cause being temporary, unstable, and changeable. They might distinguish between a temporary and unstable cause on the basis that a temporary cause is activated for a short period but will terminate at some point, while an unstable cause is subject to periodic fluctuations but will not terminate. Moreover, they might perceive differences between stable and permanent causes on the one hand and changeable causes on the other. A cause can be relatively stable and at the same time potentially changeable; for example, failure in solving a maths problem can be attributed to lack of knowledge which can be rated by an individual as rather stable but potentially changeable through learning. In conclusion, more attention should be given to the development of a more reliable measure of 
causal stability by taking into account the possible differences between the items of the CDSII's subscale of causal stability.

The finding that organisational norms of openness to change lead to higher motivation for similar tasks via expectancy of success advocates the conducive effect of such social norms to creative behaviour. Openness to change causes the formulation of high expectancy of future success leading to higher levels of intrinsic motivation for similar tasks which is regarded by Amabile (1983) as the mechanism by which social factors may effect creativity. Therefore, the the extension of the creativity model within organisational behaviour, concerning the identification of the organisational norms of individual creativity, internal cooperation, and achievement as social facilitators of creative behaviour, is supported.

In addition, the results of this study showed that organisational norms promoting centralisation of power and internal competition have a negative effect on after-failure motivation for similar tasks via their impact on expectancy of future success. This finding is additional evidence supporting the positive effect of openness to change on intrinsic motivation since it shows that the effect of organisational norms on expectancy of success differs according to our hypotheses.

Even though openness to change and resistance to change emerged as distinct factors of the construct of organisational culture (chapter 7; Xenikou \& Furnham, in press), the results of this study show that both low openness to change (absence of a facilitator of innovation) and high resistance to change (presence of an inhibitor of innovation) lead to low expectancy of future success and low after-failure motivation. The finding that low openness to change and high resistance have the same effect on expectancy of success should not be considered as evidence that the two variables are opposite poles of the same dimension rather than 
separate factors. This is the case because firstly, the two variables tend to be negatively correlated which to some extent explains the above results, and secondly, although both variables lead to low expectancy of success, there might be a substantive difference between them. Low openness to change might indicate indifference towards innovation (absence of facilitation) or active inhibition of innovation which is identical to high resistance of change. If low openness to change signified only high resistance to change the two variables of openness and resistance to change would be the opposite poles of one dimension; however, since low openness to change is not associated with high resistance when it indicates indifference to creativity, the two variables should be considered as separate factors.

At this point one should refer to the finding of study 3 that pro-creativity social norms have a positive effect on after-failure motivation via causal stability, which was interpreted as a result of pro-creativity groups experiencing failures and anti-creativity groups not experiencing failures (availability of comparison targets, see chapter 6). The elaboration of the extension of the creativity model within organisational behaviour seems to stress the importance of prior successes on perceived collective efficacy for a group to be able to maintain its members motivation to be creative following a failure event. It has to be made clear that norms promoting creativity, as well as co-operation and achievement, lead to the experience of failure since the promotion of new ideas is involved with risk taking. However, norms of openness to change are mainly related to successes since such social norms encourage members to persevere until they succeed.

In conclusion, the organisational social norms that encourage openness to change were identified as social facilitators of creative behaviour. The extension of the creativity theory proposed in chapter 6 is further elaborated by including the social norms of openness to 
change as social conditions affecting motivation to be creative at work. 
CHAPTER NINE

GENERAL DISCUSSION 


\subsection{INTRODUCTION.}

The aim of this thesis was firstly, to refine Weiner's attributional theory of motivation regarding the effect of causal stability on after-failure motivation, and secondly, to test the attributional reformulation of Amabile's creativity model, as well as the extension of Amabile's creativity theory which concerns the social facilitation of creative behaviour. In order to pursue this aim a number of hypotheses were articulated and tested. In the following section the empirical findings are presented.

A limitation of the research presented in this thesis concerns the use of self-report measures. The collection of data through self-report measures has a major drawback. Respondents are aware that they are under investigation, and may modify their responses as a result of motivational factors, such as social desirability. To the extent that these motivations bias the respondent's responses, the self-report measure will provide a distorted reflection of his/her beliefs or behaviours (Manstead \& Semin, 1992). In order to minimise the drawbacks of self-report measures it was emphasised to the respondents that their responses are anonymous. However, it is still possible that other data collection techniques (i.e., observational measure) could have led to different findings.

\subsection{SUMMARY OF EMPIRICAL FINDINGS.}

\subsubsection{Study 1 .}

The first study concerned the comparison of social learning theory (Rotter, 1966) and Weiner's (1986) attributional theory. Social learning theory suggests that locus of control is 
the causal dimension which determines motivation following a failure event. On the other hand, Weiner's theory argues that the stability of a cause rather than its locus of control is the predictor of after-failure motivation to engage in similar tasks. The aim of the study was to compare the two theoretical approaches to motivation after refining Weiner's attributional theory. The refinement of Weiner's theory identifies the initial level of motivation as a moderator variable of the relationship between causal stability and after-failure motivation. The initial level of motivation determines whether causal stability predicts subsequent motivation; more specifically, when the initial motivation to carry out a task is high, the causal stability of failure effects the level of after-failure motivation.

To compare the two theories, the direct effect of the causal dimensions of stability and locus of control on the level of after-failure motivation was tested. It was hypothesised that causal stability is a predictor of subsequent motivation provided that the initial level of motivation to engage in the task is high. The results showed that only causal stability predicted after-failure motivation provided that the initial motivation to engage in the task was high. Therefore, the refinement of Weiner's attributional theory was supported. In addition, the findings show the superiority of the refined attributional theory over social learning theory in identifying the causal dimension which determines the levels of after-failure motivation.

\subsubsection{Study 2 .}

The second study served as a preliminary task before testing one of the hypotheses of this thesis which was whether attributional style effects the process of making situational attributions. It concerned the development of a reliable and valid measure of attributional style since the relevant literature has questioned the psychometric properties of the various measures 
of attributional style, which inevitably led to questioning the concept itself. Therefore, this study addresses the issue of the measurement, as well as the conceptualisation, of the construct of attributional style.

The results indicated that attributional style for positive events and attributional style for negative events should not be considered as opposite poles of a general attributional style, but rather as separate variables. In addition, the results showed that the dimensions of stability and globality demonstrate a relatively strong cross-situational consistency and therefore, are reliable measures of the concept of attributional style. On the other hand, the dimension of internality was proven to be unreliable (did not reach the acceptable levels of internal consistency) in the current study, as well as in a number of other studies (see table 5.3). Finally, the dimension of negative globality emerged as a predictor of pessimism and confidence, and thus as a valid measure of the attributional style construct.

\subsubsection{Study 3 .}

In study three, attributional theory was utilised for the reformulation of Amabile's (1983) model of creativity. Amabile's creativity model suggests that failure in a creativity task leads to lower levels of subsequent motivation to engage in similar tasks, while its attributional reformulation argues for the moderating role of attributions (causal stability of failure) in the relationship between failure and subsequent motivation. It is hypothesised that when failure is attributed to unstable causes, after-failure motivation will not drop, whereas, when failure is attributed to stable causes, motivation will decrease provided that the initial motivation to engage in the task is high. Within the reformulation of the creativity model, the effect of attributional style on the formulation of situational attributions is addressed. In conclusion, the 
attributional reformulation of the creativity model shows the direct effect of situational attributions for failure, as well as the indirect effect of attributional style (via causal stability), on the levels of after-failure motivation to carry out similar tasks.

Study three also addresses the issue of the identification of social factors that enhance creative behaviour through the mechanism of intrinsic motivation; that is, the extension of Amabile's creativity model. Amabile and her colleagues have recently acknowledged that their research has focused on the investigation of social constraints of creativity and that future research needs to investigate the circumstances under which social facilitation of creativity might occur. It was hypothesised that pro-creativity social norms can possibly lead to the formulation of unstable attributions for failure. Social comparison mechanisms were used for explaining the hypothesised effect of pro-creativity norms on the causal stability of failure.

The results showed that failure in a creativity task per se does not lower subsequent motivation to engage in similar tasks, and that self-perceived causal stability of failure determines the level of after-failure motivation. These findings support the suggested attributional reformulation of Amabile's creativity model. Moreover, attributional style's facet of negative globality was shown to effect the formulation of situational attributions for failure; that is, a high score on attributional style's dimension of globality for negative events leads to the perception of the causes of a specific failure as stable. The attributional style facet of negative globality had an indirect effect on after-failure motivation; more specifically, a high score on negative globality leads to low levels of after-failure motivation through its impact on the formulation of stable attributions for a specific failure. On the basis of these findings, it is possible to suggest that situational attributions for failure, as well as attributional style, have an effect on motivation following a failure event. Situational attributions have a direct effect on subsequent motivation, while attributional style has an indirect effect via the 
formulation of situational attributions.

Finally, social norms regarding creativity were found to influence after-failure motivation through their effect on causal stability of failure. Pro-creativity social norms are related to unstable attributions for failure which lead to high after-failure motivation for similar tasks. Therefore, pro-creativity social norms operate as a facilitator of creative behaviour through the mechanism of intrinsic motivation. The findings support the proposed extension of Amabile's creativity theory in order to incorporate social variables that promote creative behaviour.

\subsubsection{Study 4 .}

Since there is a debate regarding whether quantitative methods should be used in the study of organisational culture, study four concerned the psychometric properties of four questionnaire measures of the concept of culture. The internal consistency and convergent validity of the questionnaires were examined. In addition, a factor analysis was carried out in order to investigate whether the dimensions (themes) of culture measured by the questionnaires could be reduced to a conceptual model of organisational culture. Finally, the factor analysis could provide some evidence concerning whether the dimensions of culture presented in the literature as facilitators of innovation (e.g., internal co-operation, achievement) or inhibitors of innovation (e.g., power-orientation, competition) are perceived by the respondents to be similar and therefore, would cluster together.

The results showed that the Organisational Culture Inventory (OCI; Cooke \& Lafferty, 1989) had higher coefficients of internal consistency (ranging from .60 to .95 ) indicating that it is the most reliable measure of culture compared to the other three. Moreover, the results 
of the correlational analysis showed that the various subscales which intend to measure the same dimension of culture were moderately correlated, providing evidence for the convergent validity of the inventories.

Finally, a readily interpretable factor structure emerged from a higher-order factor analysis of the four questionnaires' subscales. A five-dimensional model of the concept of organisational culture was supported. One of the dimensions of culture seems to be "openness to change" which contained subscales measuring task innovation, internal cooperation/support, and achievement. A second dimension of the model was labelled "resistance to change" and consisted of subscales referring to centralisation of power, competition, negativism toward the ideas of others, and perfectionism.

\subsubsection{Study 5 .}

In this study, the extension of Amabile's creativity theory tested in chapter 6 was further elaborated within organisational behaviour by examining the effect of organisational culture on the motivation to be creative. It was hypothesised that organisational norms of openness to change have a conducive effect on the motivation to be creative via their impact on causal stability of failure and expectancy of future success. In addition, since the organisational culture literature report centralisation of power and internal competition (resistance to change) to be inhibitors of innovation, the effect of resistance to change on afterfailure motivation was tested. Even though the testing of the effect of resistance to change is not directly related to the extension of Amabile's theory, which concerns the identification of social facilitators of creativity, it can provide additional evidence of the positive effect of openness to change on after-failure motivation if it is proven to be negatively related to after- 
failure motivation.

The findings provided partial support of the hypotheses of this study. None of the hypotheses regarding the relations of perceived causal stability with the other variables was supported. Since perceived causal stability of failure was shown to have unacceptable levels of internal reliability (alpha $=.48$ ), it can be argued that the hypothesised relationships involving causal stability could not be tested in this study.

All the other hypotheses were supported by the results. Expectancy of future success was shown to determine the levels of subsequent motivation; that is, high expectancy of success was associated with high levels of after-failure motivation. Moreover, organisational norms of openness to change had a positive effect on the expectancy of future success. Since expectancy of success emerged as a significant predictor of shift in intrinsic motivation and openness to change is positively related to expectancy of success, it is possible to suggest that openness to change has an indirect effect on levels of after-failure motivation via its impact on the formulation of expectations for future success. Finally, organisational norms of resistance to change were shown to lead to low expectancy of success; therefore, it was shown that resistance to change has a negative indirect effect on after-failure motivation through its impact on expectancy of success.

The findings of this study suggest that the organisational norms of openness to change (the co-existence of creativity, co-operation, and achievement norms) lead to high levels of after-failure motivation; they are, therefore, conducive to creative behaviour, since intrinsic motivation is, according to Amabile (1983), the mechanism through which a number of social factors influence creativity. The identification of an organisational culture which promotes openness to change as a social facilitator of creativity supports the proposed extension of Amabile's theory of creativity within organisational behaviour. 


\subsection{SITUATIONAL ATTRIBUTIONS FOR FAILURE AND SUBSEQUENT MOTIVATION.}

One of the questions addressed by this thesis concerns whether situational attributions for failure determine subsequent motivation to engage in similar tasks. The findings support the proposed refinement of Weiner's attributional theory, as well as the superiority of the refined attributional theory of Weiner to social learning theory. Therefore, it is possible to conclude that when failure has been encountered, the stability of the provided causes determines motivation to carry out similar tasks provided that the initial interest in the task is high. In other words, the stability of situational attributions does effect motivation given that there is initial motivation to engage in the task.

\subsubsection{The refined attributional theory of Weiner.}

Weiner's attributional theory of motivation proposes that situational attributions moderate the effect of failure on subsequent levels of motivation. More specifically, when a failure is attributed to unstable causes subsequent motivation remains at the same levels or might even increase, while stable attributions for failure lead to lower motivation for similar tasks.

In this thesis the refinement of Weiner's attributional theory is put forward, which is an attempt to explain the findings of a number of studies either contradicting or failing to confirm the theory's predictions. The refinement concerns the identification of the initial level of motivation to engage in a task as a moderator variable of the relation between causal 
stability and subsequent motivation; that is, causal stability predicts after-failure motivation for similar tasks provided that the initial interest to carry out the task is high. In other words, when a person fails in a task which he/she initially considers to be uninteresting, an attributional process is not likely to be activated and even if it is, the stability of the causes of failure does not predict future motivation. This is mainly the case because there is no after-failure motivation to change a potentially changeable cause when a task is not appealing in the first place; actually, after-failure motivation can drop as a result of the general negative effect of failure.

The results supported the refinement of Weiner's attributional theory since causal stability emerged as a predictor of shift in motivation to engage in similar tasks only if the initial level of motivation was high. Therefore, it is possible to conclude that causal stability of failure determines subsequent levels of motivation provided that the initial motivation to carry out the task is high.

\subsubsection{Social learning theory vs. the refined attributional theory of Weiner.}

There are two main attributional theories which suggest that situational attributions influence motivation, namely, social learning theory (Rotter, 1966) and Weiner's (1986) attributional theory. Although both of these theories suggest that situational attributions influence motivation, they make different predictions regarding which is the specific causal dimension determining the levels of motivation. Social learning theory (Rotter, 1966) proposes that locus of control is the causal dimension which predicts subsequent motivation, whereas Weiner's (1986) attributional theory suggests that causal stability is the predictor. In this thesis a refinement of Weiner's attributional theory was proposed and evidence was provided that the 
refined theory is superior to Weiner's theory in the case of failure.

A comparison of the social learning theory and the refined attributional theory of Weiner was conducted. The findings showed that locus of control did not emerge as a predictor of after-failure motivation indicating that the attribution of failure to internal or external causes does not influence the levels of subsequent motivation. On the other hand, causal stability of failure was proven to determine after-failure motivation provided that the initial level of motivation to engage in the task was high. These findings advocate the superiority of the refined attributional theory of Weiner to social learning theory concerning their predictions of the effect of attributions on after-failure motivation.

\subsubsection{The issue of the measurement of causal stability.}

Within Weiner's attributional paradigm, the measurement of causal stability involves the a priori classification by the researcher of various causes into the dimension of causal stability. The subjects are normally asked to rate the provided causes (e.g., Weiner, Nierenberg, \& Goldstein, 1976) or to answer forced-choice questions containing all possible pairs of the given causes. In either method the four major causes, namely, ability, difficulty of the task, effort, and luck (e.g., Kovenklioglu \& Greenhaus, 1978) are used; the causes of ability and task difficulty are classified as stable causes, while effort and luck are considered to be unstable causes.

There are two main disadvantages related to these methods of measuring causal stability. Firstly, the fact that the subjects are given a list of possible causes of an event and are asked to choose one is, according to Wimer and Kelley (1982), a rather passive way of making attributions inhibiting the clearer and fuller understanding of the meaning of a cause. The 
second disadvantage concerns the possibility that misclassification of the causes takes place; for example, ability is not always perceived as a stable cause as in the case that learning can facilitate the development of ability. Since the possible misclassification of causes has been used as an explanation of the inadequacy of causal stability to predict motivation (Weiner, 1983; Winefield, Tiggemann, \& Winefield, 1992), it is important to make sure that such misclassification does not occur.

In this thesis to overcome the problems related to the measurement of causal stability, respondents were asked to provide their own causes of events and to rate these causes on three 7-point items designed by McAuley, Duncan, and Russell (1992) to measure causal stability. The items were measuring whether a cause is permanent or temporary, stable or unstable, and finally, unchangeable or changeable. Even though the scale was reported by the constructors to have acceptable levels of alpha reliability $($ alpha $=.67)$ and it reached acceptable alphas in two of the studies of this thesis (study 1 : alpha $=.76$ and study $3:$ alpha $=.79$ ), the measurement of causal stability was shown to be unreliable in study five (study 5 : alpha $=.48$ ). Thus, although the problem of the misclassification of causes on the dimension of stability is handled by asking the respondents to rate the cause on a scale measuring stability, the items comprising McAuley, Duncan, and Russell's (1992) stability scale are not always perceived by the raters as similar. Thus, it is important that future research addresses the issue of developing a reliable measure of the construct of causal stability.

In conclusion, the measurement of perceived causal stability (respondents rating the causes on a stability scale) is preferred to the classification of the various causes by the researcher as either stable or unstable, simply because the latter can possibly lead to the misclassification of the causes. However, more attention should be paid to the psychometric properties of the McAuley, Duncan, and Russell's (1992) causal stability scale and especially, 
the issue of its internal reliability.

\subsection{SITUATIONAL ATTRIBUTIONS, ATTRIBUTIONAL STYLE, AND MOTIVATION.}

After demonstrating the effect of situational attributions on motivation, the question concerning the joint effect of situational attributions and attributional style on the levels of motivation was addressed. It was hypothesised that when situational information about the occurrence of an event is ambiguous, attributional style influences the formulation of situational attributions which, in turn, determine the levels of subsequent motivation. The findings showed that attributional style has an indirect effect on motivation via its impact on situational attributions (perceived causal stability) supporting the above hypothesis.

\subsubsection{The conceptualisation and measurement of attributional style.}

An important preliminary task before testing the hypothesis regarding the indirect effect of attributional style on motivation via causal stability, was to address issues of the conceptualisation, as well as the actual measurement, of the concept of attributional style.

Regarding the conceptualisation of attributional style, the findings show that attributional style for positive events and attributional style for negative events are distinct variables. Therefore, Abramson, Seligman, and Teasdale's (1978), as well as Seligman's (1990), definition of a "vulnerable" or "pessimistic" attributional style as the attribution of negative events to internal, stable, and global causes, and the attribution of positive events to external, 
unstable, and specific ones, is not accurate. Moreover, since it was demonstrated that the dimension of internality shows systematically low levels of alpha reliability, this dimension should not be included in the conceptualisation of attributional style. Finally, the finding that negative globality is a valid measure of pessimism and confidence suggests that a pessimistic attributional style should be defined as the degree to which one holds generalised low expectations for future performance.

On the grounds of re-defining the concept of attributional style, a proposition for a reliable and valid measure of the concept was suggested. Firstly, the dimension of internality needs to be omitted since it has proven to be unreliable; secondly, attributions for negative events should be used instead of both positive and negative; and thirdly, the measurement of the concept can be based on negative globality which was shown to be reliable (high levels of internal consistency) and valid (predictor of pessimism and confidence).

The findings of the second study suggest that the common practice of using composite scores, that is, adding up the scores on internality, stability, and globality, should not be followed. This is the case because internality was shown to be a consistently unreliable measure of attributional style and the validity of the dimension of stability was not demonstrated since it failed to predict either pessimism or confidence. Thus, findings of previous studies within the attributional style literature which are based on the usage of composite scores may be questioned.

9.4.2 The relation between situational attributions, attributional style, and motivation.

It has been suggested in the literature (Anderson, 1983; Mikulincer, 1990) that 
situational attributions eliminate the effect of attributional style on motivation. That is, when failure has been encountered attributional style influences subsequent motivation if there is absence of any attribution-eliciting information, while the presence of such information eliminates any effect of attributional style on motivation. Thus, situational attributions have a dominant effect on motivation suppressing the effect of attributional style.

However, this thesis argues that the above finding might be the result of situational information being rather indisputable since the researchers were experimentally controlling the formulation of situational attributions by providing information that would lead to stable or unstable attributions. Therefore, it is proposed in this thesis that the competing relationship between situational attributions and attributional style regarding their effect on motivation might not take place if attribution-eliciting information is ambiguous. In this case the attributional style might effect the perception of the situational information leading to situational attributions, which in turn determines motivation. In other words, there may be a joint effect of attributional style and situational attributions on motivation.

The findings of the third study support the hypothesis that attributional style has an indirect effect on motivation via situational attributions. It is, therefore, possible to suggest that attributional style, as a result of accumulated past experiences, effects the way information is perceived, and consequently, the formulation of situational attributions. Thus, attributional style is a mechanism that makes the formulation of situational attributions possible even when information is rather limited. Furthermore, if attributional style can compensate for the lack of information, then it might be related to a process of making biased attributions. 


\subsection{THE ATTRIBUTIONAL REFORMULATION OF AMABILE'S CREATIVITY MODEL.}

The attributional reformulation of the creativity model was supported by the findings of the third study. Amabile's (1983) prediction that failure in a creativity task leads to lower levels of motivation was disconfirmed; it was demonstrated that following failure in a creativity task there were significantly more people reporting the same or higher levels of motivation than lower levels of motivation. In addition, the attribution of failure to stable or unstable causes was shown to determine subsequent motivation to engage in similar tasks. Finally, attributional style had an effect on the formulation of causal stability, which in turn determined the levels of after-failure motivation.

Therefore, Amabile's creativity model needs to incorporate causal stability as the moderator of the failure effect on the levels of intrinsic motivation. When the outcome of the creative process is failure (stage 5 of Amabile's creativity model) the attribution of this event to stable causes leads to lower levels of intrinsic motivation, while an unstable attribution leads to the same or higher motivation for similar tasks. Moreover, attributional style's facet of globality, that is, the extent to which one holds generalised negative expectations for future events, influences the formulation of stable or unstable attributions, and thus, has an indirect effect on intrinsic motivation.

The attributional reformulation of Amabile's creativity model, concerning the joint effect of situational attributions and attributional style on after-failure motivation to be creative, was supported. This finding advocates the interference of cognitions in the process of reinforcement. The behaviouristic approach suggests that a negative event (failure) leads to decrements in motivation, while a positive event leads to increments in subsequent motivation. 
On the other hand, cognitive oriented researchers argue that negative or positive reinforcement does not directly effect motivation since cognitions, such as beliefs, values, attitudes, and attributions, interfere. The reformulation of Amabile's creativity model is an attempt to put forward the cognitive approach and to emphasise the moderating role of attributions in the relationship between negative reinforcement and motivation. The results are actually in favour of the cognitive perspective since failure per se did not lower the levels of motivation and causal stability was shown to predict subsequent motivation for similar tasks.

One could argue that the findings of this study emerged as a result of respondents engaging in a retrospective explanation of past events. Therefore, it is possible that attributions were shown to affect after-failure motivation because respondents tend to construct a rational sequence of behaviours when asked to explain a past event, rather than because they do actually behave in the way that they report. This alternative interpretation of the findings is, however, a speculation that needs to be addressed in future research.

The attributional perspective on creativity seems to open novel ways of approaching such a broad and complex area as the area of creativity. Future research could address the issue that there might exist stages regarding the effect of failure on intrinsic motivation for similar tasks. It is possible that in an early stage failing in a creativity process leads to experiencing lack of motivation to engage in similar tasks (stage 1), but motivation is restored in a second stage (stage 2) where an attributional process is initiated, as far as the outcome of the attributional process is explaining the event in terms of an unstable cause. Therefore, it is possible that although intrinsic motivation is actually sensitive to the effect of failure, the attributions act as the mechanism which restores the temporary lack of motivation to engage in similar tasks. 


\subsection{THE EXTENSION OF AMABILE'S "SOCIAL PSYCHOLOGY OF CREATIVITY."}

Amabile's "social psychology of creativity" concerns the investigation of the effect of a number of social factors on creative behaviour via their impact on intrinsic motivation to engage in a creativity task. However, the social psychology of creativity has focused on the detrimental effect of a number of social variables, such as evaluation and competition, on intrinsic motivation, while neglecting any positive effect that social factors might have on intrinsic motivation. In order to fill this gap within the social psychology of creativity, Amabile and her colleagues suggested that future research should investigate the social facilitators of creative behaviour.

This thesis proposed an extension of the social psychology of creativity based on the identification of social variables which promote intrinsic motivation to be creative. It was suggested that social norms placing a high value on creativity might facilitate creative behaviour through the mechanism of causal stability. More specifically, members of groups which encourage creativity might tend to attribute their failures in a creativity task to unstable causes, whereas members of anti-creativity groups might attribute their failures to stable causes. The findings of this thesis supported the hypothesis that group norms regarding creativity affect the levels of after-failure motivation through their impact on causal stability. Therefore, it is possible to suggest that pro-creativity social norms do have an indirect positive effect on after-failure intrinsic motivation to be creative and can, therefore, be identified as a social facilitator of creativity.

In explaining the reasons why pro-creativity social norms lead to high after-failure motivation via their effect on causal stability of failure, the theory of social comparison was 
utilised. When failure has been encountered, people look for comparison targets (other people who failed in the same or similar tasks in the past) in order to reduce the anxiety related with the negative effect of failure. Groups with pro-creativity norms are tolerant of failure which they perceive as a necessary side-effect of the process of developing creative ideas, and therefore, their members find comparison targets easily. The fact that members can compare their own failures to the failures of others leads to the perception of the causes of failure as unstable, since other members who failed in the past eventually managed to succeed. Future research could investigate whether the process of social comparison is the mechanism that explains the relation between social norms regarding creativity and causal stability of failure.

In addition, the extension of Amabile's creativity theory was further elaborated within organisational behaviour. It was shown that an organisational culture promoting creativity, internal co-operation, and achievement, namely, openness to change, leads to high after-failure motivation through its effect on the expectancy of future success. Organisational norms promoting openness to change do characterise innovative groups which believe in their collective efficacy, that is, their capability to perform well (Lawson \& Ventriss, 1992). Therefore, a culture of openness to change is related to high degrees of perceived collective efficacy leading to high expectancy of future success even though failure was encountered. Future research needs to examine in more detail the effect of innovative groups' collective efficacy on members intrinsic motivation to be creative.

A limitation of the current research was that the extension of the social psychology of creativity was theorised and tested only for the case of failing in a creativity task. Thus, the findings demonstrated the facilitating effect of creativity social norms and organisational culture on creative behaviour for the case of failure, but did not provide any evidence of social facilitation of creativity when the outcome of the creativity process is success or partial 
success. It is possible that the role of attributions will remain central if the outcome is unexpected or the task is important to the person, since Weiner (1986) has suggested that an attributional process is likely to be initiated when the outcome is negative, unexpected, or the task is important. However, future research is needed in order for these questions to be answered.

In conclusion, an extension of Amabile's theory was put forward in this thesis. The findings supported the identification of pro-creativity social norms and an organisational culture which promotes openness to change as social facilitators of creativity via the mechanism of intrinsic motivation. 


\section{REFERENCES}

Abramson, L.Y., Seligman, M.E.P., \& Teasdale, J.D. 1978. Learned helplessness in humans: Critique and reformulation. Journal of Abnormal Psychology, 87(1), 49-74.

Abramson, L.Y., Metalsky, G.I., \& Alloy, L.B. 1989. Hopelessness depression: A theorybased subtype of depression. Psychological Review, 96(2), 358-372.

Ahrens, A.H. \& Haaga, D.A.F. 1993. The specificity of attributional style and expectations to positive and negative affectivity, depression, and anxiety. Cognitive Therapy and Research, 17(1), 83-98.

Aiken, M., Bacharach, S.B., \& French, J.L. 1980. Organisational structure, work process, and proposal making in administrative bureaucracies. Academy of Management Journal, vol. 23(4), 631-652.

Allen, R.F. \& Dyer, F.J. 1980. A tool for tapping the organizational unconscious. Personnel Journal, March, 192-199.

Amabile, T.M. 1979. Effects of external evaluation on artistic creativity. Journal of Personality and Social Psychology, 37, 221-233.

Amabile, T.M. 1982. Children's artistic creativity: Detrimental effects of competition in a field setting. Personality and Social Psychology Bulletin, 8, 573-578.

Amabile, T.M. 1983. The social psychology of creativity: A componential conceptualisation. Journal of Personality and Social Psychology, 45, 357-376.

Amabile, T.M. 1985. Motivation and creativity: Effects of motivational orientation on creative writers. Journal of Personality and Social Psychology, 48, 393-399

Amabile, T.M. 1988. A model of creativity and innovation in organisations. In D.G. Gardner and L.L. Cummings (eds.), Research in organisational behaviour, vol. 10. 
Greenwich, CN: JAI Press.

Amabile, T.M. \& Gitomer, J. 1984. Children's artistic creativity: Effects of choice in task materials. Personality and Social Psychology Bulletin, 10, 209-215.

Amabile, T.M., Hennessey, B.M., \& Grossman, B.S. 1986. Social influences on creativity: The effect of contracted-for reward. Journal of Personality and Social Psychology, $50,14-23$.

Amabile, T.M., Goldfarb, P., \& Brackfield, S.C. 1990. Social influences on creativity: Evaluation, coaction, and surveillance. Creativity Research Journal, Vol. 3(1), 6-21. Anderson, C.A. 1983. Motivational and performance deficits in interpersonal settings: The effect of attributional style. Journal of Personality and Social Psychology, 45(5), $1136-1147$.

Anderson, C.A, Jennings, D.L., \& Arnoult, L.H. 1988. Validity and utility of the attributional style construct at a moderate level of specificity. Journal of Personality and Social Psychology, 55(6), 979-990.

Antaki, C. 1985. Ordinary explanation in conversation: Causal structures and their defence. European Journal of Social Psychology, 15, 213-230.

Argyle, M. \& Henderson, M. 1985. The Anatomy of relationships. London: Heinemann. Atkinson, J.W. 1964. An introduction to motivation. Princeton, NJ: Van Nostrand Bem. Bradbury, T.N. \& Fincham, F.D. 1988. Assessing spontaneous attributions in marital interaction: Methodological and conceptual considerations. Journal of Social and Clinical Psychology, 7(2-3), 122-130.

Beck, A.T., Weissman, A., Lester, D. \& Trexter, L. 1974. The measurement of pessimism: The hopelessness scale. Journal of Consulting and Clinical Psychology, 42(6), 861865. 
Bem, D. 1972. Self-perception theory. In L. Berkowitz (ed.), Advances in experimental social psychology, Vol. 6. New York: Academic Press.

Brewin, C.R. \& Shapiro, A. 1984. Beyond locus of control: Attribution of responsibility for positive and negative outcomes. British Journal of Psychology, 75, 43-49

Brewin, C.R. \& Furnham, A. 1986. Attributional versus preattributional variables in selfesteem and depression: A comparison and test of learned helplessness theory. Journal of Personality and Social Psychology, 50(5), 1013-1020.

Brewin, C.R. 1988. Cognitive foundations of Clinical Psychology. London: Lawrence Erlbaum.

Burns, M.O. \& Seligman, M.E.P. 1989. Explanatory style across the life span: Evidence for stability over 52 years. Journal of Personality and Social Psychology, 56(3), 471477.

Burnside, R.M. 1990. Improving corporate climates for creativity. In M.A. West and J.L. Farr (eds.), Innovation and creativity at work: Psychological and organisational strategies. New York: John Wiley \& Sons.

Child, D. 1977. Psychology and the teacher. London: Holt, Rinehart/Winston.

Cooke, R.A. \& Lafferty, J.C. 1989. Organisational Culture Inventory. Plymouth, MI: Human Synergistics.

Cooke, R.A. \& Rousseau, D.M. 1988. Behavioural norms and expectations: A quantitative Approach to the assessment of organisational culture. Group and Organisation Studies, Vol 13(3), 245-273.

Corr, P.J. \& Gray, J.A. 1994. The role of attributional style, socialisation, and cognitive abilities in insurance selling: An argument for interaction regression models in validity estimation. Paper presented at the BPS Occupational Section Conference, Brighton. 
Covington, M.V. \& Omelich, C.L. 1979. Are causal attributions causal? A path analysis of the cognitive model of achievement motivation. Journal of Personality and Social Psychology, 37, 1487-1504.

Covington, M.V. \& Omelich, C.L. 1984. The trouble with pitfalls: A reply to Weiner's critique of attributional research. Journal of Educational Psychology, 76(6), 11991213.

Cutrona, C.E., Russell, D. \& Jones, R.D. 1985. Cross-situational consistency in causal attributions: Does attributional style exist? Journal of Personality and Social Psychology, 47(5), 1043-1058.

Dalal, A.K. \& Sethi, A. 1988. An attributional study of high and low need achievers in India. The Journal of Social Psychology, 128(1), 55-64.

Damanpour, F. 1991. Organisational innovation: A meta-analysis of effects of determinants and moderators. Academy of Management Journal, vol. 34(3), 555-590.

Davis, G.A. 1986. Creativity is forever. Dubuque, IA: Kendall/Hunt.

Deal, T.E. \& Kennedy, A.A. 1982. Corporate cultures: The rites and rituals of corporate life. Reading, Mass: Addison/Wesley.

Degot, V. 1987. Corporate culture and the concept of rationality in corporate models. International Studies of Management and Organisations, 17, 19-39.

Duncker, K. 1945. On problem solving. Psychological Monographs, 58(270).

D'Zurilla, T.J. \& Nezu, A.M. 1990. Development and preliminary evaluation of the Social Problem-Solving Inventory. Psychological Assessment: A Journal of Consulting and Clinical Psychology, 2, 156-163.

D'Zurilla, T.J. \& Sheedy, C.F. 1991. Relation between social problem-solving ability and subsequent level of psychological stress in college students. Journal of Personality 
and Social Psychology, 61(5), 841-846.

Eisenberger, R. \& Selbst, M. 1994. Does reward increase or decrease creativity? Joumal of Personality and Social Psychology, 66(6), 1116-1127.

Feather, N.T. 1982. Human values and the prediction of action: An expectancy-valence analysis. In N.T. Feather (ed.), Expectations and actions: Expectancy-value models in Psychology. NJ: Lawrence Erlbaum Associates, Inc.

Feldman, S.P. 1988. How organisational culture can affect innovation. Organisational Dynamics, vol. 17(1), 57-68.

Fontaine, G. 1974. Social comparison and some determinants of expected personal control and expected performance in a novel task situation. Journal of Personality and Social Psychology, 29, 487-496.

Furnham, A. 1982a. Explanations for unemployment in Britain. European Journal of Social Psychology, 12, 335-352.

Furnham, A. 1982b. Why are the poor always with us? Explanations for poverty in Britain. British Journal of Social Psychology, 21, 311-322.

Furnham, A. \& Bochner, S. 1986. Culture Shock: Psychological Reactions to Unfamiliar Environments. London: Methuen.

Furnham, A., Sadka, V., \& Brewin, C. 1992. The development of an occupational attributional style questionnaire. Journal of Organisational Behaviour, 13, 27-39.

Furnham, A. \& Gunter, B. 1993. Corporate Assessment: Auditing a company's personality. London: Routledge.

Furnham, A. \& Steele, H. 1993. Measuring locus of control: A critique of general, children's, health-and work-related locus of control questionnaires. British Journal of Psychology, 84, 443-479. 
Furnham, A., Brewin, C.R. \& O'Kelly, H. 1994. Cognitive style and attitudes to work. Human Relations, 47(12), 1509-1521.

Gibbons, F.X. 1986. Social comparison and depression: Company's effect on misery. Journal of Personality and Social Psychology, 51, 140-148.

Glaser, R. 1983. The Corporate Culture Survey. Bryn Mawr, PA: Organisational Design and Development.

Glassman, E. 1986. Managing for creativity: Back to basics in R \& D. $R \& D$ Management, $16,175-183$.

Glick, W.H. 1985. Conceptualizing and measuring organisational and psychological climate: Pitfalls in multilevel research. Academy of Management Review, 1985, Vol.10(3), 601-616.

Goldsmith, R.E. \& Matherly, T.A.1987. Adaptation-innovation and creativity: A replication and extension. British Journal of Social Psychology, 26, 79-82.

Gordon, G.G. 1985. The relationship of corporate culture to industry sector and corporate performance. In R.H. Kilman, M.J. Saxton \& R. Serpa (eds.), Gaining control of the corporate culture. San Fransisco: Jossey-Bass.

Graham, S. 1984. Communicating sympathy and anger to black and white children: The cognitive (attributional) consequences of affective cues. Journal of Personality and Social Psychology, 47, 40-54.

Gregory, K.L. 1983. Native-view paradigms: Multiple cultures and culture conflicts in organisations. Administrative Science Quarterly, 28, 359-376.

Hage, J. \& Aiken, M. 1970. Social change in complex organisations. New York: Random House.

Hampden-Turner, C. 1990. Corporate culture: From vicious to virtuous circles. London: 
The Random Century.

Handy, C. 1986. Understanding organisations. Harmondsworth: Penguine Books.

Harrison, R. 1972. Understanding your organisation's character. Harvard Business Review, May-June, 119-128.

Heaven, P.C.L. 1994. Occupational attributional style and attitudes to work: An Australian study. Australian Psychologist, 29, 57-61.

Hofstede, G. 1986. Culture's consequences: International differences in work-related values. Bevery-Hills, CA: Sage.

Hofstede, G., Neuijen, B., Ohayv, D.D., \& Sanders, G. 1990. Measuring organisational cultures: A qualitative study across twenty cases, Administrative Science Quarterly, $35,286-316$.

Holden, K.B. \& Rotter, J.B. 1962. A nonverbal measure of extinction in skill and chance situations. Journal of Experimental Psychology, 63, 519-520.

Hull, J.G. \& Mendolia, M. 1991. Modelling the relations of attributional style, expectancies, and depression. Journal of Personality and Social Psychology, 61(1), 85-97.

Jöreskog, K.G. \& Sörbom, D. 1993. Lisrel VIII user's reference guide. Chicago, IL: Scientific Software International, Inc.

Kanter, R.M. 1983. The change masters. London: George Allen \& Unwin.

Kasof, J. 1995. Explaining creativity: The attributional perspective. Creativity Research Journal, 8(4), 311-366.

Kelley, H.H. 1967. Attribution theory in social psychology. In D. Levin (ed.), Nebraska Symposium of Motivation, vol. 15. Lincoln, NB: University of Nebraska Press.

Kelley, H.H. 1983. Perceived causal structures. In J. Jaspars, F.D. Fincham, \& M. Hewstone (eds.), Attribution theory and research: Conceptual, developmental, and social 
dimensions. London: Academic Press.

Kilman, R.H. \& Saxton, M. J. 1983. The Kilman-Saxton Culture-Gap Survey. Pittsburgh, PA: Organisational Design Consultants.

Kilman, R.H., Saxton, M.J., \& Serpa, R. 1985. Gaining control of the corporate culture. San Fransisco: Jossey/Bass.

Kilmann, R.H. \& Thomas, K.W. 1977. Developing a forced-choice measure of conflicthandling behavior: The "MODE" instrument. Educational and Psychological Measurement, 37, 309-325.

King, N. 1990. Innovation at work: The research literature. In M.A. West and J.L. Farr (eds.), Innovation and creativity at work: Psychological and organisational strategies. New York: John Wiley \& Sons.

Kirton, M.J. 1976. Adaptors and innovators: A description and measure. Journal of Applied Psychology, 6, 622-629.

Kirton, M.J. 1978. Have adaptors and innovators equal levels of creativity? Psychological Reports, 42, 695-698.

Kovenklioglu, G. \& Greenhaus, J.H. 1978. Causal attributions, expectations, and task performance. Journal of Applied Psychology, 63(6), 698-705.

Lawson, R.B. \& Ventriss, C.L. 1992. Organisational change: The role of organisational culture and organisational learning. Psychological Record, 42, 205-219.

Lemyre, L. \& Smith, P.M. 1985. Intergroup discrimination and self-esteem in the minimal intergroup paradigm. Journal of Personality and Social Psychology, 49, 660-670.

Levine, J.M. \& Green, S.M. 1984. Acquisition of relative performance information. Personality and Social Psychology Bulletin, 10, 385-393.

Livingstone, S.M. \& Lunt, P.K. 1989. Debating drunk driving: The construction of causal 
explanations in television discussion programs. Paper presented at BPS Social Section Conference, Bristol.

Lorsch, J.W. 1985. Strategic myopia: Cultures as an invisible barrier to change. In R.H. Kilman, M.J. Saxton, \& R. Serpa (eds.) Gaining Control of the Corporate Culture. San Fransisco: Jossey/Bass.

MacKinnon, D.W. 1965. Personality and the realization of creative potential. American Psychologist, 20, 273-281.

Manstead, A.S.R. \& Semin, G.R. 1992. Methodology in social psychology: Turning ideas into actions. In M. Hewstone, W.Stroebe, J.P. Codol, and G. Stephenson (eds.), Introduction to social psychology. Oxford: Blackwell.

Margulies, N. \& Raia, A. 1978. Conceptual foundations of organisational development. New York: McGraw/Hill.

Martin, H.J. 1985. Managing specialised corporate cultures. In R.H. Kilman, M.J. Saxton, \& R. Serpa (eds.) Gaining Control of the Corporate Culture. San Fransisco: Jossey/Bass.

Martin, J. and Siehl, C. 1983. Organisational culture and counterculture: An uneasy symbiosis. Organisational Dynamics, Autumn.

McAuley, E., Duncan, T.E., \& Russell, D.W. 1992. Measuring causal attributions: The revised Causal Dimension Scale. Personality and Social Psychology Bulletin, 18(5), $566-573$.

McCrae, R.R. \& Costa, P.T.,Jr. 1987. Validation of the five-factor model of personality across instruments and observers. Joumal of Personality and Social Psychology, 52, 81-90.

McMahan, I.D. 1973. Relationships between causal attributions and expectancy of success. 
Journal of Personality and Social Psychology, 28(1), 108-114.

Mednick, S. 1962. The associative basis of the creative process. Psychological Review, 69, $220-232$.

Michael, R. 1979. How to find-and keep-creative people. Research Management, September, 43-45.

Mikulincer, M. 1990. Joint influence of prior beliefs and current situational information on stable and unstable attributions. The Journal of Social Psychology, 130(6), 739-753.

Miller, F.D., Smith, E.R, \& Uleman, J. 1981. Measurement and interpretation of situational and dispositional attributions. Journal of Experimental Social Psychology, Vol. $17(1), 80-95$.

Morey, N.C. \& Luthans, F. 1985. Refining the displacement of culture and the use of scenes and themes in organisational studies. Academy of Management Review, 10(2), 219229.

Newell, A., Shaw, J.C., \& Simon, H.A. 1963. The process of creative thinking. In H.E. Gruber (ed.), Contemporary approaches to creative thinking. New York: Atherton.

Nunnally, J.C. 1978. Psychometric Theory. New York: McGraw-Hill.

Nystrom, H. 1979. Creativity and innovation. New York: John Wiley.

Nystrom, H. 1990. Organisational innovation. In M.A. West and J.L. Farr (eds.), Innovation and creativity at work: Psychological and organisational strategies. New York: John Wiley \& Sons.

O'Reilly, C.A., Chatman, J. \& Caldwell, D.F. 1988. People, jobs and organisational culture. Working paper, University of California, Berkeley.

O'Reilly, C.A., Chatman, J. \& Caldwell, D.F. 1991. People and Organisational Culture: A profile comparison approach to assessing person-organization fit. Academy of 
Management Journal, Vol. 34(3), 487-516.

Peters, T.J. \& Waterman, R.H. 1982. In research of excellence: Lessons from America's best-run companies. New York: Harper \& Raw.

Peterson, C. 1991. The meaning and measurement of explanatory style. Psychological Inquiry, 2, 1-10.

Peterson, C., Semmel, A., von Baeyer, C., Abramson, L.Y., Metalsky, G.I., \& Seligman, M.E.P. 1982. The Attributional Style Questionnaire. Cognitive Therapy and Research, 6(3), 287-300.

Peterson, C. \& Villanova, P. 1988. An Expanded Attributional Style Questionnaire. Journal of Abnormal Psychology, 97(1), 87-89.

Phares, E.J. 1957. Expectancy changes in skill and chance situations. Journal of Abnormal and Social Psychology, 54, 339-342.

Potter, C.C. 1989. What is culture: And can it be useful for organisational change agents? Leadership and Organisation Development Journal, Vol. 10(3), 17-24.

Quinn, R.E. 1988. Beyond rational management. San Francisco, CA: Jossey-Bass.

Reichers, A.E. \& Schneider, B. 1990. Climate and culture: An evolution of constructs. In B. Schneider (ed.), Organisational climate and culture. San Francisco: Jossey/Bass.

Rotter, J.B. 1966. Generalised expectancies for internal versus external control of reinforcement. Psychological Monographs: General and Applied, 80(1), 1-28.

Rotter, J.B., Seeman, M., \& Liverant, S. 1962. Internal versus external control of reinforcement: A major variable in behaviour theory. In N.F. Washburne (ed.), Decisions, values, and groups, Vol. 2. London: Pergamon Press.

Rousseau, D.M. 1990. Quantitative assessment of organisational culture: The case for multiple methods. In B. Schneider (ed.), Organisational climate and culture. San 
Francisco: Jossey/Bass.

Rust, J. 1991. Rapid Personality Questionnaire: Technical manual. London: Personality Systems Limited.

Sashkin, M. 1991. Pillars of excellence: Organizational beliefs questionnaire. Carmarthen: Organizational Design and Development.

Saxton, M.J. 1987. The validation of the Kilman-Saxton culture-gap survey to test a theory of work group cultural norms and an exploration of a nomological network of culture. Unpublished doctoral dissertation. University of Pittsburgh.

Schein, E.H. 1984. Suppose we took culture seriously. Academy of Management OD Newsletter, Summer, 2-3ff.

Schein, E.H. 1985. Organisational culture and leadership. San Francisco: Jossey/Bass.

Schein, E.H. 1986. What you need to know about organisational Culture. Training and development Journal, January, 30-33.

Schein, E.H. 1990. Organisational culture. American Psychologist, 45(2), 109-1 19.

Schwartz, H.M. \& Davis, S.M. 1981. Matching corporate culture and business strategy. Organisational Dynamics, Summer, 30-48.

Seligman, M.E.P. 1990. Leamed optimism: How to change your mind and your life. London: Pocket Books.

Seligman, M.E.P \& Schulman, P. 1986. Explanatory style as a predictor of productivity and quitting among life insurance sales agents. Journal of Personality and Social Psychology, 50(4), 832-838.

Siehl, C. \& Martin, J. 1988. Mixing qualitative and quantitative methods. In M.O. Jones, M.D. Moore, and R.C. Snyder, Inside organisations: Understanding the human dimension. London: Sage. 
Smircich, L. 1982. Organizations as shared meanings. In L.R. Pondy, P.Frost, G.Morgan and T.C. Dandringe (eds.), Organisational symbolism. Greenwich CI: JAI Press.

Spector, P. 1988. Development of the Work Locus of Control Scale. Journal of Ocupational Psychology, 61, 335-340.

Staw, B.M. 1984. Organisational behaviour: A review and reformulation of Field's outcome variables. Annual Review of Psychology, 35, 627-666.

Torrance, E.P. 1988. The nature of creativity as manifest in its testing. In R.S. Sternberg (ed.), The nature of creativity. Cambridge: Cambridge University Press.

Weiner, B. 1980. Human motivation. New York: Holt, Reinhart/Winston.

Weiner, B. 1983. Some methodological pitfalls in attributional research. Journal of Educational Psychology, 75(4), 530-543.

Weiner, B. 1986. An attributional theory of motivation and emotion. New York: Springer/Verlag.

Weiner, B. 1991. An attributional look at explanatory style. Psychological Inquiry, 2(1), 4344.

Weiner, B., Nierenberg, R., \& Goldstein, M. 1976. Social learning (locus of control) versus attributional (causal stability) interpretations of expectancy of success. Journal of Personality, 44, 52-68.

West, M.A. 1987. Role innovation in the world of work. British Journal of Social Psychology, 26, 305-315

West, M.A. 1989. Innovation among health care professionals. Social Behaviour, 4, 15-30.

West, M.A. \& Farr, J.L. 1990. Innovation and creativity at work: Psychological and organisational strategies. New York: Wiley.

West, M.A. \& Farr, J.L. 1990. Innovation at work. In M.A. West and J.L. Farr (eds.), 
Innovation and creativity at work: Psychological and organisational strategies. New York: Wiley.

West, M.A. \& Anderson, N. 1992. Innovation, cultural values, and the management of change in British hospitals. Work \& Stress, vol. 6(3), 293-310.

Williams, A., Dobson, P., \& Walters, M. 1989. Changing culture: New organisational approaches. London: IPM.

Wimer, S. \& Kelley, H.H. 1982. An investigation of the dimensions of causal attribution. Journal of Personality and Social Psychology, 43(6), 1142-1162.

Winefield, A.H., Tiggemann, M., \& Winefield, H.R. 1992. Unemployment distress, reasons for job loss and causal attributions for unemployment in young people. Journal of Occupational and Organisational Psychology, 65, 213-218.

Wood, J.V. 1989. Theory and research concerning social comparison of personal attributes. Psychological Bulletin, 106(2), 231-248.

Xenikou, A. \& Fumham, A. A correlational and factor analytic study of four questionnaire measures of organisational culture. Human Relations, in press.

Xenikou, A., Furnham, A. \& McCarrey, M.W. Attributional style for negative events: A proposition for a more reliable and valid measure of attributional style. British Journal of Psychology, in press.

Zaltman, G., Duncan, R., \& Holbek, J. 1973. Innovations and organisations. London: John Wiley \& Sons.

Zautra, A.J., Guenther, R.T., \& Chartier, G.M. 1985. Attributions for real and hypothetical events: Their relation to self-esteem and depression. Journal of Abnormal Psychology, 94(4), 530-540. 
Appendix 
Questionnaire of Study One 
You are presented with three stories. Please read the first one carefully and write a brief summary in the space provided. Then continue by reading the second story and writing a brief summary of it. Finally, read the third one and write a summary of it.

Story 1:

One day a rich man found that his win cellar was empty. So he sent out messengers to announce a generous offer. The first person to bring the rich man a barrel of wine would be given a brick of solid gold. However, the offer would expire at sundown.

Two wine merchants heard the news. Each had a horse-drawn cart loaded with large barrels of wine. They both set out for the duke's palace at once. An hour before sundown they came to a place where the bridge had been washed out by a ranging river. The first merchant drove his horses and cart into the flood in a desperate attempt to reach the other side. But the horses were already exhausted and could not fight the current. The cart overturned, and the horses, wine, and driver were washed away.

The second merchant tried a different tactic. He poured the wine out of all but one of his barrels, and lashed them together to form a raft; then he loaded the one full barrel, a horse, and himself on top. He set the raft adrift and floated downstream. In a few minutes the raft came to rest on the shore in front of the town where the rich man lived. The merchant disembarked, loaded the wine barrel on the horse, and led it to the rich man's house. He arrived just as the sun was setting, and collected the gold brick as a reward for his efforts. 
Story 2:

A small country was controlled by a dictator. The dictator ruled the country from a strong fortress. The fortress was situated in the middle of the country, surrounded by farms and villages. Many roads radiated outward from the fortress like spokes on a wheel. To celebrate the anniversary of his rise to power, the dictator ordered his general to conduct a full-scale military parade. On the morning of the anniversary, the general's troops were gathered at the head of one of the roads leading to the fortress, ready to march. However, a lieutenant brought the general a disturbing report. The dictator was demanding that this parade had to be more impressive than any previous parade. He wanted his army to be seen and heard at the same time in every region of the country. Furthermore, the dictator was threatening that if the parade was not sufficiently impressive he was going to strip the general of his medals and reduce him to the rank of private. But it seemed impossible to have a parade that could be seen throughout the whole country.

The general, however, knew just what to do. He divided his army up into small groups and dispatched each group to the head of a different road. When all was ready he gave the signal, and each group marched down a different road. Each group continued down its road to the fortress, so that the entire army finally arrived together at the fortress at the same time. In this way, the general was able to have the parade seen and heard through the entire country at once, and thus please the dictator. 
Story 3:

Once there were identical twins who were continually playing pranks on their family, friends and teachers. The annual school picnic was always a big event for the twins. There were races and other athletic events in which the twins won lots of prizes. One year a new student arrived who was a star runner. The twins wanted to win the main event: the 2-mile race through the woods behind the school. So they secretly devised a plan which would enable them to outdo the newcomer.

The day of the race arrived. Each runner was to pick his own path through the woods to a clearing, where a teacher stood posted to determine the winner. One twin entered the race, while the other excused himself on the grounds that he had hurt his leg in an earlier broadjumping event. The race began and the students rushed into the woods. The twin rushed into the woods and waited until the others passed out of sight. Then he went back to the school using a path hidden from the picnic area. Shortly after, the other twin, who had been hiding behind a rock near the finish line of the race, burst out and ran into the clearing ahead of the other runners. The teacher named him the winner and marvelled at the speed of his running. Next year the twins switched places and thereafter maintained their status on this event. 
-The problem that follows requires some creativity for its solution so you should not feel inhibited about making any suggestions that come to mind.

-Please try to solve the problem on your own without asking the opinion of the people sitting next to you as this is a procedure of individual and not group problem solving. Write down the solution(s) in the space provided.

\section{The Radiation Problem}

Suppose you are a doctor faced with a patient who has a malignant tumour in his stomach. It is impossible to operate on the patient, but unless the tumour is destroyed the patient will die. There is a kind of ray that can be used to destroy the tumour. If the rays reach the tumour all at once at a sufficiently high intensity, the tumour will be destroyed. Unfortunately, at this intensity the healthy tissue that the rays pass through on the way to the tumour will also be destroyed. At lower intensities the rays are harmless to healthy tissue, but they will not affect the tumour either. What type of procedure might be used to destroy the tumour with the rays, and at the same time avoid destroying the healthy tissue? 
Now please answer the following questions by circling the appropriate response.

1. Did you know the solution to the radiation problem prior to the experiment?

YES

NO

2. Do you think that this problem solving task was interesting?

$\begin{array}{lllllllll}\begin{array}{l}\text { Not } \\ \text { Interesting }\end{array} & 1 & 2 & 3 & 4 & 5 & 6 & 7 & \begin{array}{l}\text { Very } \\ \text { Interesting }\end{array}\end{array}$

3. How challenging did you perceive this problem solving task to be?

$\begin{array}{lllllllll}\text { Not at all } & 1 & 2 & 3 & 4 & 5 & 6 & 7 & \text { Very }\end{array}$

Challenging

Challenging 


\section{SUBJECT'S CODE:}

Prior research has shown that $49 \%$ of people who are presented with the radiation problem come up with an adequate solution. The possible solutions to the problem are the following:

A. Reduce the intensity of rays on the way to tumour by applying many low intensity rays from different directions simultaneously, so that the healthy tissue is not damaged and the tumour is destroyed.

B. Avoid contact between rays and healthy tissue by sending high-intensity rays through an open route (e.g. oesophagus).

Therefore if you compare the solution(s) that you suggested to the adequate solutions you can see that you failed to give a correct solution to the problem either by giving a partial solution, an inappropriate one or no solution at all. Please write down the major reason of this failure in the space provided.

MAJOR REASON FOR FAILURE:

Think about the reason you have written above. The items below concern your impressions or opinions of this cause of your failure. Circle one number for each of the following questions.

Is the cause something:

1. Manageable

by you

2. Permanent

3. You can regulate

4. Stable over time

5. Over which you you have power

6. Unchangeable $\begin{array}{lll}7 & 6 & 5\end{array}$

4

4

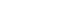

Not manageable by you

$\begin{array}{llllllll}7 & 6 & 5 & 4 & 3 & 2 & 1 & \text { Temporary }\end{array}$

76

5

43

2

1 You cannot regulate

Variable over time

$\begin{array}{llllllll}7 & 6 & 5 & 4 & 3 & 2 & 1 & \begin{array}{l}\text { Over which } \\ \text { you have no } \\ \text { power }\end{array}\end{array}$

$\begin{array}{llllllll}7 & 6 & 5 & 4 & 3 & 2 & 1 & \text { Changeable }\end{array}$


Now please answer the following questions.

1. How challenging would you find solving another problem of this type?

$\begin{array}{lllllllll}\text { Not } & 1 & 2 & 3 & 4 & 5 & 6 & 7 & \text { Very }\end{array}$

Challenging Challenging

2. How interesting would you find solving another problem of this type?

$\begin{array}{lcccccccc}\text { Not } 1 & 2 & 3 & 4 & 5 & 6 & 7 & \begin{array}{l}\text { Very } \\ \text { Interesting }\end{array} \\ \text { Interesting } & & & & & & & \end{array}$


Questionnaire of Study Three 
In the course of doing a Ph.D we all receive positive and negative feedback about our work. Please try to remember one specific occasion when you presented a new idea for running an experiment (or conducting a study) to your supervisor and she/he rejected it. Write down the reason(s) of this failure in the space provided.

REASON(S) FOR FAILURE:

Think about the reason(s) you have written above. The items below concern your impressions or opinions of this cause or causes of your failure. Circle one number for each of the following questions.

Is the cause something:

$\begin{array}{lllllllll}\text { 1. Permanent } & 7 & 6 & 5 & 4 & 3 & 2 & 1 & \text { Temporary }\end{array}$

$\begin{array}{llllllllll}\text { 2. That reflects an } & 7 & 6 & 5 & 4 & 3 & 2 & 1 & \text { Reflects an }\end{array}$ aspect of yourself aspect of the situation

3. Stable

$7 \quad 6$

5

43

2

Variable over time

4. Over which you have power

$7 \quad 6$

43

2

1
over time

6. Unchangeable

7

6

5

4

32

21

Over which you have no power

Changeable 
Now please answer the following questions.

1. Did the rejection of your new idea have an effect on how challenging you perceive your work to be?

$\begin{array}{lllllllll}\text { Less } & 7 & 6 & 5 & 4 & 3 & 2 & 1 & \begin{array}{l}\text { More } \\ \text { Challenging }\end{array} \\ \text { Challenging } & & & & & & & & \end{array}$

2. Did the rejection of your new idea by your supervisor have an effect on how interesting you perceive your work to be?

$\begin{array}{lllllllll}\text { Less } & 7 & 6 & 5 & 4 & 3 & 2 & 1 & \begin{array}{l}\text { More } \\ \text { Interesting }\end{array} \\ \text { Interesting } & & & & & & & & \end{array}$

3. Do you believe that it is important to be creative in your work?

$\begin{array}{lllllllll}\text { It is } & 7 & 6 & 5 & 4 & 3 & 2 & 1 & \begin{array}{l}\text { It is not } \\ \text { Important }\end{array} \\ \text { Important } & & & & & & & \end{array}$

5. Do you think it is important to carry out experiments/studies that are original?

$\begin{array}{lllllllll}\text { It is } & 7 & 6 & 5 & 4 & 3 & 2 & 1 & \begin{array}{l}\text { It is not } \\ \text { Important }\end{array} \\ \text { Important } & & & & & & & \end{array}$

6. Does your supervisor directly or indirectly insists on the originality of your experiments/studies?

$\begin{array}{lllllllll}\begin{array}{l}\text { She/he } \\ \text { insists on } \\ \text { Originality }\end{array} & 7 & 6 & 5 & 4 & 3 & 2 & 1 & \begin{array}{l}\text { She/he doesn't } \\ \text { insist on } \\ \text { Originality }\end{array}\end{array}$




\section{HYPOTHETICAL SITUATIONS QUESTIONNAIRE}

As Ph.D. students we all experience a number of negative outcomes concerning our work. In this section you are asked to vividly imagine you are in a series of hypothetical situations and to write the single most likely cause of the hypothetical event. You will then be asked to rate that cause on 2 scales and answer a question about the importance of the situation.

Please vividly imagine you are in the situation where your supervisor commented on your literature review as incomplete. Write down the single most likely cause of this negative event in the space below.

NOW RATE THE CAUSE THAT YOU WROTE ABOVE ON THE FOLLOWING SCALES:

\section{PLEASE KEEP IN MIND THAT YOU ARE RATING THE CAUSE THAT YOU WROTE ABOVE AND NOT THE HYPOTHETICAL EVENT PROVIDED.}

1. In the future when your supervisor comments on your work, will this negative cause again influence what happens?

$\begin{aligned} & \text { Will never } \begin{array}{l}7 \\ \text { again influence }\end{array} \\ & \text { agall always influence }\end{aligned}$
What happens

what happens

2. Is this negative cause something that just affects this situation or does it influence other areas of your life?

$\begin{array}{lclllllll}\text { Influences } & 7 & 6 & 5 & 4 & 3 & 2 & 1 & \begin{array}{l}\text { Influences all areas } \\ \text { of my life }\end{array} \\ \text { just this situation } & & & & & & \end{array}$

\section{NOW PLEASE ANSWER A QUESTION ABOUT THE HYPOTHETICAL EVENT:}

1. How important would this situation be if it really happened to you?

$\begin{array}{lllllllll}\begin{array}{l}\text { Not at all } \\ \text { important }\end{array} & 7 & 6 & 5 & 4 & 3 & 2 & 1 & \begin{array}{l}\text { Extremely } \\ \text { important }\end{array} \\ \end{array}$


Please vividly imagine you submitted a paper to a conference and it was rejected. Write down the single most likely cause of this negative event in the space below.

NOW RATE THE CAUSE THAT YOU WROTE ABOVE ON THE FOLLOWING SCALES:

PLEASE KEEP IN MIND THAT YOU ARE RATING THE CAUSE THAT YOU WROTE ABOVE AND NOT THE HYPOTHETICAL EVENT PROVIDED.

1. In the future when your supervisor comments on your work, will this negative cause again influence what happens?

$\begin{array}{lllllllll}\text { Will never } & 7 & 6 & 5 & 4 & 3 & 2 & 1 & \text { Will always influence }\end{array}$ again influence what happens what happens

2. Is this negative cause something that just affects this situation or does it influence other areas of your life?

$\begin{array}{lllllllll}\text { Influences } & 7 & 6 & 5 & 4 & 3 & 2 & 1 & \text { Influences all areas }\end{array}$ just this situation of my life

\section{NOW PLEASE ANSWER A OUESTION ABOUT THE HYPOTHETICAL EVENT:}

1. How important would this situation be if it really happened to you?

$\begin{array}{lllllllll}\begin{array}{l}\text { Not at all } \\ \text { important }\end{array} & 7 & 6 & 5 & 4 & 3 & 2 & 1 & \begin{array}{l}\text { Extremely } \\ \text { important }\end{array} \\ \end{array}$


Imagine that the last experiment/study that you carried out failed to provide any significant results. Write down the single most likely cause of this negative event in the space below.

NOW RATE THE CAUSE THAT YOU WROTE ABOVE ON THE FOLLOWING SCALES:

PLEASE KEEP IN MIND THAT YOU ARE RATING THE CAUSE THAT YOU WROTE ABOVE AND NOT THE HYPOTHETICAL EVENT PROVIDED.

1. In the future when your supervisor comments on your work, will this negative cause again influence what happens?

$\begin{array}{lrlllllll}\begin{array}{l}\text { Will never } \\ \text { again influence }\end{array} & 6 & 5 & 4 & 3 & 2 & 1 & \begin{array}{l}\text { Will always influence } \\ \text { what happens }\end{array} \\ \text { Wh }\end{array}$
what happens

2. Is this negative cause something that just affects this situation or does it influence other areas of your life? $\begin{array}{lclllllll}\text { Influences } \begin{array}{c}7 \\ \text { just this situation }\end{array} & 6 & 5 & 4 & 3 & 2 & 1 & \begin{array}{l}\text { Influences all areas } \\ \text { of my life }\end{array}\end{array}$

\section{NOW PLEASE ANSWER A OUESTION ABOUT THE HYPOTHETICAL EVENT:}

1. How important would this situation be if it really happened to you?

$\begin{array}{lllllllll}\begin{array}{l}\text { Not at all } \\ \text { important }\end{array} & 7 & 6 & 5 & 4 & 3 & 2 & 1 & \begin{array}{l}\text { Extremely } \\ \text { important }\end{array} \\ \text { imporat }\end{array}$


Please vividly imagine that you developed a new methodology that was severely criticised by experts in your field. Write down the single most likely cause of this negative event in the space below.

NOW RATE THE CAUSE THAT YOU WROTE ABOVE ON THE FOLLOWING SCALES:

PLEASE KEEP IN MIND THAT YOU ARE RATING THE CAUSE THAT YOU WROTE ABOVE AND NOT THE HYPOTHETICAL EVENT PROVIDED.

1. In the future when your supervisor comments on your work, will this negative cause again influence what happens?

$\begin{array}{lllllllll}\text { Will never } & 7 & 6 & 5 & 4 & 3 & 2 & 1 & \text { Will always influence }\end{array}$ again influence what happens what happens

2. Is this negative cause something that just affects this situation or does it influence other areas of your life?

$\begin{array}{lllllllll}\text { Influences } & 7 & 6 & 5 & 4 & 3 & 2 & 1 & \text { Influences all areas }\end{array}$ just this situation of my life

\section{NOW PLEASE ANSWER A OUESTION ABOUT THE HYPOTHETICAL EVENT:}

1. How important would this situation be if it really happened to you?

$\begin{array}{lllllllll}\begin{array}{l}\text { Not at all } \\ \text { important }\end{array} & 7 & 6 & 5 & 4 & 3 & 2 & 1 & \begin{array}{l}\text { Extremely } \\ \text { important }\end{array} \\ \end{array}$


Please vividly imagine that you presented your pilot study in front of other postgrads and departmental staff and there was negative feedback. Write down the single most likely cause of this event in the space below.

$* * * * * * * * * * * * * * * * * * * * * * * * * * * * * * * * * * * * * * * * * * * * * * * * * * * * * * * * * * * * * * * * * * * * * * * * * *$

NOW RATE THE CAUSE THAT YOU WROTE ABOVE ON THE FOLLOWING SCALES:

PLEASE KEEP IN MIND THAT YOU ARE RATING THE CAUSE THAT YOU WROTE ABOVE AND NOT THE HYPOTHETICAL EVENT PROVIDED.

1. In the future when your supervisor comments on your work, will this negative cause again influence what happens?

$\begin{array}{lllllllll}\text { Will never } & 7 & 6 & 5 & 4 & 3 & 2 & 1 & \text { Will always influence }\end{array}$ again influence what happens

what happens

2. Is this negative cause something that just affects this situation or does it influence other areas of your life?

$\begin{array}{lllllllll}\text { Influences } & 7 & 6 & 5 & 4 & 3 & 2 & 1 & \text { Influences all areas }\end{array}$

just this situation

of my life

\section{NOW PLEASE ANSWER A QUESTION ABOUT THE HYPOTHETICAL EVENT:}

1. How important would this situation be if it really happened to you?

$\begin{array}{lllllllll}\text { Not at all } & 7 & 6 & 5 & 4 & 3 & 2 & 1 & \text { Extremely }\end{array}$

important important


Please vividly imagine you can't get all the work done that your supervisor expects of you. Write down the single most likely cause of this negative event in the space below.

NOW RATE THE CAUSE THAT YOU WROTE ABOVE ON THE FOLLOWING SCALES:

PLEASE KEEP IN MIND THAT YOU ARE RATING THE CAUSE THAT YOU WROTE ABOVE AND NOT THE HYPOTHETICAL EVENT PROVIDED.

1. In the future when your supervisor comments on your work, will this negative cause again influence what happens?

$\begin{array}{lrlllllll}\begin{array}{l}\text { Will never } \\ \text { again influence }\end{array} & 6 & 5 & 4 & 3 & 2 & 1 & \begin{array}{l}\text { Will always influence } \\ \text { what happens }\end{array} \\ \text { wat }\end{array}$
what happens

2. Is this negative cause something that just affects this situation or does it influence other areas of your life?

$\begin{array}{lllllllll}\text { Influences } & 7 & 6 & 5 & 4 & 3 & 2 & 1 & \text { Influences all areas }\end{array}$ just this situation of my life

\section{NOW PLEASE ANSWER A OUESTION ABOUT THE HYPOTHETICAL EVENT:}

1. How important would this situation be if it really happened to you?

\begin{tabular}{|c|c|c|c|c|}
\hline $\begin{array}{l}\text { Not at all } \\
\text { important }\end{array}$ & 7 & 6 & 5 & 4 \\
\hline
\end{tabular}


Please vividly imagine that your supervisor rejected your idea for the next experiment/study that you designed. Write down the single most likely cause of this negative event in the space below.

NOW RATE THE CAUSE THAT YOU WROTE ABOVE ON THE FOLLOWING SCALES:

PLEASE KEEP IN MIND THAT YOU ARE RATING THE CAUSE THAT YOU WROTE ABOVE AND NOT THE HYPOTHETICAL EVENT PROVIDED.

1. In the future when your supervisor comments on your work, will this negative cause again influence what happens?

$\begin{array}{lrlllllll}\begin{array}{l}\text { Will never } \\ \text { again influence }\end{array} & 6 & 5 & 4 & 3 & 2 & 1 & \begin{array}{l}\text { Will always influence } \\ \text { what happens }\end{array} \\ \text { Wal }\end{array}$ what happens

2. Is this negative cause something that just affects this situation or does it influence other areas of your life?

$\begin{array}{lcccccccc}\text { Influences } & 7 & 6 & 5 & 4 & 3 & 2 & 1 & \text { Influences all areas }\end{array}$ just this situation of my life

\section{NOW PLEASE ANSWER A QUESTION ABOUT THE HYPOTHETICAL EVENT:}

1. How important would this situation be if it really happened to you?

$\begin{array}{lllllllll}\begin{array}{l}\text { Not at all } \\ \text { important }\end{array} & 7 & 6 & 5 & 4 & 3 & 2 & 1 & \begin{array}{l}\text { Extremely } \\ \text { important }\end{array} \\ \text { imporat }\end{array}$


Please vividly imagine that you applied for a grant to attend a course that would be very useful for your research and you didn't get it. Write down the single most likely cause of this negative event in the space below.

NOW RATE THE CAUSE THAT YOU WROTE ABOVE ON THE FOLLOWING SCALES:

PLEASE KEEP IN MIND THAT YOU ARE RATING THE CAUSE THAT YOU WROTE ABOVE AND NOT THE HYPOTHETICAL EVENT PROVIDED.

1. In the future when your supervisor comments on your work, will this negative cause again influence what happens?

$\begin{array}{lrlllllll}\begin{array}{l}\text { Will never } \\ \text { again influence }\end{array} & 6 & 5 & 4 & 3 & 2 & 1 & \begin{array}{l}\text { Will always influence } \\ \text { what happens }\end{array} \\ \text { What hapens }\end{array}$

what happens

2. Is this negative cause something that just affects this situation or does it influence other areas of your life?

$\begin{array}{lllllllll}\text { Influences } & 7 & 6 & 5 & 4 & 3 & 2 & 1 & \text { Influences all areas }\end{array}$ just this situation of my life

\section{NOW PLEASE ANSWER A QUESTION ABOUT THE HYPOTHETICAL EVENT:}

1. How important would this situation be if it really happened to you?

$\begin{array}{lllllllll}\begin{array}{l}\text { Not at all } \\ \text { important }\end{array} & 7 & 6 & 5 & 4 & 3 & 2 & 1 & \begin{array}{l}\text { Extremely } \\ \text { important }\end{array} \\ \text { impon }\end{array}$


Please vividly imagine that you had sent a paper to a journal for publishing and the reviewers rejected it. Write down the single most likely cause of this negative event in the space below.

NOW RATE THE CAUSE THAT YOU WROTE ABOVE ON THE FOLLOWING SCALES:

PLEASE KEEP IN MIND THAT YOU ARE RATING THE CAUSE THAT YOU WROTE ABOVE AND NOT THE HYPOTHETICAL EVENT PROVIDED.

1. In the future when your supervisor comments on your work, will this negative cause again influence what happens?

$\begin{array}{lllllllll}\text { Will never } & 7 & 6 & 5 & 4 & 3 & 2 & 1 & \text { Will always influence }\end{array}$ again influence what happens

what happens

2. Is this negative cause something that just affects this situation or does it influence other areas of your life?

$\begin{array}{lcccccccc}\text { Influences } & 7 & 6 & 5 & 4 & 3 & 2 & 1 & \begin{array}{l}\text { Influences all areas } \\ \text { of my life }\end{array}\end{array}$
just this situation of my life

\section{NOW PLEASE ANSWER A OUESTION ABOUT THE HYPOTHETICAL EVENT:}

1. How important would this situation be if it really happened to you?

$\begin{array}{lllllllll}\begin{array}{l}\text { Not at all } \\ \text { important }\end{array} & 7 & 6 & 5 & 4 & 3 & 2 & 1 & \begin{array}{c}\text { Extremely } \\ \text { important }\end{array} \\ \end{array}$


Please vividly imagine that you sent a report to your sponsor about the state of your research and they reply that it seems to go very slowly. Write down the single most likely cause of this negative event in the space below.

NOW RATE THE CAUSE THAT YOU WROTE ABOVE ON THE FOLLOWING SCALES:

PLEASE KEEP IN MIND THAT YOU ARE RATING THE CAUSE THAT YOU WROTE ABOVE AND NOT THE HYPOTHETICAL EVENT PROVIDED.

1. In the future when your supervisor comments on your work, will this negative cause again influence what happens?

$\begin{array}{lllllllll}\text { Will never } & 7 & 6 & 5 & 4 & 3 & 2 & 1 & \text { Will always influence }\end{array}$ again influence what happens

what happens

2. Is this negative cause something that just affects this situation or does it influence other areas of your life?

$\begin{array}{lllllllll}\text { Influences } & 7 & 6 & 5 & 4 & 3 & 2 & 1 & \text { Influences all areas }\end{array}$ just this situation of my life

\section{NOW PLEASE ANSWER A QUESTION ABOUT THE HYPOTHETICAL EVENT:}

1. How important would this situation be if it really happened to you?

$\begin{array}{lllllllll}\text { Not at all } & 7 & 6 & 5 & 4 & 3 & 2 & 1 & \text { Extremely }\end{array}$

important important


Questionnaire of Study Five 


\section{HYPOTHETICAL SITUATION}

In the following paragraph you are presented with a hypothetical scenario that is taking place in the company where you are currently employed. Please put yourself in the situation, as vividly as possible.

The management of the company is concerned about the improvement of customer service. In order to collect as many ideas about customer service as possible, the management has decided to use "suggestion boxes." Any individual employee who is interested and feels challenged by the task of finding new ways to serve customers, has the opportunity to write an extensive proposal of her/his ideas and put it in the suggestion box. Each of the proposed ideas is discussed thoroughly by the management and is either accepted or rejected for application. The proposals are anonymous, so that the hierarchical position, occupation, or status of the employees won't interfere in the process of management decision making. However, each proposal has a code on it and these codes are used for the announcement of whether a proposal was accepted or rejected. Employees whose proposals are accepted for application are expected to contact the management.

Now imagine that you proposed a new idea for the improvement of customer service and it was rejected. What was the main cause of this rejection? (write down in the space provided).

Main Cause of the rejection of your idea

Think about the cause you have written above. The items below concern your impressions or opinions of this cause. Circle one number for each of the following questions.

Is the cause something:

$\begin{array}{lllllllll}\text { 1. Permanent } & 7 & 6 & 5 & 4 & 3 & 2 & 1 & \text { Temporary } \\ \begin{array}{l}\text { 2. Manageable } \\ \text { by you }\end{array} & 7 & 6 & 5 & 4 & 3 & 2 & 1 & \begin{array}{l}\text { Not manageable } \\ \text { by you }\end{array} \\ \begin{array}{l}\text { 3. Stable } \\ \text { over time }\end{array} & 7 & 6 & 5 & 4 & 3 & 2 & 1 & \begin{array}{l}\text { Variable } \\ \text { over time }\end{array}\end{array}$


$\begin{array}{lllllllll}\text { 4. Unchangeable } & 7 & 6 & 5 & 4 & 3 & 2 & 1 & \text { Changeable }\end{array}$

Now please answer the following questions about your reactions to the hypothetical situation.

After the rejection of your idea:

1. Would it become more or less challenging to look for new ways to improve the company's operation?

$\begin{array}{lllllllll}\text { More } & 7 & 6 & 5 & 4 & 3 & 2 & 1 & \text { Less }\end{array}$

Challenging

Challenging

2. Would it become more or less interesting to look for new ways to improve the company's operation?

$\begin{array}{lllllllll}\begin{array}{l}\text { More } \\ \text { Interesting }\end{array} & 7 & 6 & 5 & 4 & 3 & 2 & 1 & \begin{array}{l}\text { Less } \\ \text { Interesting }\end{array}\end{array}$

3. Would you expect that next time you propose a new idea it will be accepted for application?

$\begin{array}{lllllllll}\text { Will be } & 7 & 6 & 5 & 4 & 3 & 2 & 1 & \text { Won't be }\end{array}$

Accepted Accepted

The following questions do not concern any aspects of the scenario.

They refer to your attitudes towards organisational climate.

1. Are you interested in finding new ways to improve the way your job is being done?

$\begin{array}{lllllllll}\text { Very } & 7 & 6 & 5 & 4 & 3 & 2 & 1 & \begin{array}{l}\text { Not at all } \\ \text { Interested }\end{array} \\ \text { Interested } & & & & & & & & \end{array}$

